\title{
Neutrino phenomenology in the flavored NMSSM without domain wall problems
}

\author{
M. A. Ouahid, ${ }^{*}$ M. A. Loualidi $\odot,{ }^{\dagger}$ R. Ahl Laamara, ${ }^{\ddagger}$ and E. H. Saidi ${ }^{\S}$ \\ LPHE-Modeling and Simulations, Faculty of Science, Mohammed V University in Rabat, 10090 Rabat, Morocco \\ and Centre of Physics and Mathematics, CPM, Faculty of Science, Rabat 10090, Morocco
}

(Received 24 September 2020; accepted 29 November 2020; published 21 December 2020)

\begin{abstract}
We propose a next-to-minimal supersymmetric Standard Model (NMSSM) extended by an $\mathbb{A}_{4} \times \boldsymbol{Z}_{3}$ flavor symmetry and three right-handed neutrinos providing a detailed study of the neutrino sector and a solution to the domain wall problem. In this proposal, neutrino masses are generated through Type I seesaw mechanism while the mixing angles are described by the trimaximal mixing realized using the NMSSM singlet $S$ and only two flavon fields. The phenomenology of neutrino parameters is studied for normal and inverted mass hierarchies. In particular, we numerically evaluated the observables related to neutrino masses and mixing, namely, $\sum m_{i}, m_{e e}, m_{\nu_{e}}$, and $\delta_{C P}$ where we find that the ranges of $m_{e e}$ and $m_{\nu_{e}}$ are accessible by current and future experiments while the obtained ranges of $\sum m_{i}$ and $\delta_{C P}$ lie within the current experimental data. Another attractive feature we discussed in this paper is the circumvention of the domain wall problem induced by the spontaneous breaking of the $A_{4} \times Z_{3}$ discrete symmetry. We first showed that the domain walls in the charged lepton sector occur at high energy scale leading to unproblematic domain walls, while in the neutrino sector they are inevitable. Then, to solve this problem, we reconsidered the well-known approach that relies on the explicit breaking of the discrete symmetry through the insertion of Planck-suppressed operators induced by supergravity.
\end{abstract}

DOI: 10.1103/PhysRevD.102.115023

\section{INTRODUCTION}

Physics beyond the standard model (SM) of elementary particles has been widely explored after the experimental discovery of neutrino oscillations [1-3], and of the Higgs boson with a mass of about $125 \mathrm{GeV}[4,5]$. One of the most studied extensions of the SM is the minimal supersymmetric Standard Model [6-8] (MSSM) which predicts the lightest Higgs boson-among the five it contains - to be lighter than the $Z$-boson. Thus, large quantum corrections are required in order to reach the mass of the discovered Higgs boson. Although the MSSM solves many problems encountered in the SM such as the hierarchy problem and the gauge coupling unification, it undergoes a naturalness concern related to the mass term involving the up and down Higgs doublets $\mu H_{u} H_{d}$. In order to generate a convenient electroweak symmetry breaking, the $\mu$-parameter which has

\footnotetext{
*mohamedamine_ouahid@um5.ac.ma

†mr.medamin@gmail.com

*ahllaamara@gmail.com

h-saidi@fsr.ac.ma
}

Published by the American Physical Society under the terms of the Creative Commons Attribution 4.0 International license. Further distribution of this work must maintain attribution to the author(s) and the published article's title, journal citation, and DOI. Funded by SCOAP. a dimension of mass must be of the order of the SUSY breaking scale $M_{\text {SUSY }}$. However, since the MSSM might be an effective theory that originates from a more fundamental high energy theory with some cutoff scale such as the GUT scale $M_{\mathrm{GUT}}$ or the Planck scale $M_{\mathrm{Pl}}$, the question that arises is why is the scale of $\mu$ far below these scales? This is the so-called the $\mu$-problem [9].

The most simple way to deal with the $\mu$-problem as well as some of the other shortcomings of the MSSM is by introducing an extra hyperchargeless singlet chiral superfield $S$ which gives rise to the well known next-to-minimal supersymmetric Standard Model [10-13]. In the latter, the $\mu$-term is replaced by the Yukawa coupling $\lambda \mathrm{SH}_{u} H_{d}$, where after the scalar component of the chiral superfield $S$ acquires a vacuum expectation value (VEV) of the order of $M_{\text {SUSY }}$, an effective $\mu$-term- $\mu_{\text {eff }}=\lambda\langle S\rangle$-with the appropriate order is generated, thus solving the $\mu$-problem of the MSSM. Moreover, since the superpotential of the NMSSM is scale invariant, the old $\mu$-term is excluded by an accidental $\mathcal{Z}_{3}$ discrete symmetry under which all the chiral superfields $\hat{\Phi}$ of the NMSSM transform as $\hat{\Phi} \rightarrow e^{2 \pi i / 3} \hat{\Phi}$ [14]. However, the rise of this discrete symmetry group $\mathcal{Z}_{3}$ is not without weakness from the cosmological perspective; indeed, the spontaneous breaking of the $\mathcal{Z}_{3}$ symmetry leads to the creation of cosmological domain walls (DWs) in the early Universe [15]; that is different $\mathcal{Z}_{3}$ ground states created during the electroweak phase transition [16,17]. 
Nevertheless, there are different ways suggested in the literature to avoid the DW problem among which we mention: (a) the breaking of $\mathcal{Z}_{3}$ symmetry taking place before the end of inflation [18,19]; and (b) taking into account the supergravitational effect by adding nonrenormalizable terms to the superpotential to break explicitly the $\mathcal{Z}_{3}$ leading to favor one of the three $\mathcal{Z}_{3}$ vacua over the others $[14,20]$. Notice by the way that the annihilation of DWs has been also suggested as a plausible source of the gravitational waves (GWs) as discussed in different extensions of the SM, see for instance [21-23]. This annihilation would constitute an interesting aspect of DWs especially after the recent observation of these GWs at the LIGO and the Virgo experiments [24], which could offer a way for investigating the existence of DWs in the early Universe.

Aside from these features related to the scalar sector, the $\mathrm{SM}$ as well as its supersymmetric extensions predict massless neutrinos, thus further extensions are required to explain the results of the neutrino oscillation experiments and hence, the observed mixing angles and the tiny neutrino masses. Recent data from these experiments give hints on the mass hierarchy, the $C P$ violating $(C P V)$ phase $\delta_{C P}$ and nonmaximal atmospheric mixing $\theta_{23}$ where two different octants of $\theta_{23}$ are allowed; the lower octant (LO) with $\theta_{23}<\pi / 4$ and the higher octant (HO) with $\theta_{23}>\pi / 4$. The values of neutrino oscillation parameters $\theta_{i j}, \Delta m_{i j}^{2}$ and $\delta_{C P}$ can be found in the latest global fit analyses [25-29]. From the theoretical point of view, one of the most wellknown ways to generate such tiny neutrino masses is through type-I seesaw mechanism which implies the introduction of heavy right-handed Majorana neutrinos to the SM or any of its supersymmetric extensions [30-35]. However, the seesaw mechanism does not allow us to explain the neutrino mixing nor to determine the neutrino mass hierarchy. In this regard, non-Abelian discrete flavor symmetries have been used extensively in recent years to describe the family structure of both leptons and quarks, in particular, these symmetries are known to produce mixing patterns compatible with large mixing angles of the lepton sector such as the tribimaximal mixing (TBM) matrix which corresponds to a unitary matrix of the form [36]

$$
U_{\mathrm{TBM}}=\left(\begin{array}{ccc}
\sqrt{\frac{2}{3}} & \frac{1}{\sqrt{3}} & 0 \\
-\frac{1}{\sqrt{6}} & \frac{1}{\sqrt{3}} & \frac{1}{\sqrt{2}} \\
-\frac{1}{\sqrt{6}} & \frac{1}{\sqrt{3}} & -\frac{1}{\sqrt{2}}
\end{array}\right)
$$

However, while the TBM matrix is consistent with the large solar $\theta_{12}$ and atmospheric $\theta_{23}$ angles, it is in conflict with the nonzero reactor $\theta_{13}$ angle discovered in 2012 [37-39]. Nevertheless, the TBM matrix is still considered as a good first order approximation while small deviations are required to accommodate the small value of $\theta_{13}$. These deviations are usually realized by introducing higher dimensional effective operators while the leading TBM contribution is produced by the seesaw mechanism. In the same manner, several nonAbelian discrete groups have been used in the literature to generate the deviation from TBM and provided a successful description of all the neutrino mixing angles; see for instance Table 3 of Ref. [40] and Refs. [41-54]. Moreover, there are additional unanswered questions relevant to neutrino physics such as whether neutrinos are Dirac or Majorana particles and the issue concerning the absolute neutrino mass scale. In this regard, many nonoscillation experiments such as beta decay $[55,56]$ and neutrinoless double beta decay [57-60] experiments have been proposed to probe the mass scale of neutrinos. The latter would also prove lepton number violation and establish the Majorana nature of neutrinos; for a recent review, see [61]. In addition, cosmological observations can probe the sum of neutrino masses $\sum m_{i}=m_{1}+m_{2}+m_{3}$ where the current upper bound given by the Planck collaboration is $\sum m_{i}<0.17 \mathrm{eV}$ [62].

In this paper, we aim to achieve two main objectives: First, we study the neutrino masses and mixing in the context of a flavored NMSSM prototype (FNMSSM) with discrete symmetry group $G_{\mathrm{f}}=\mathbb{A}_{4} \times \boldsymbol{Z}_{3}$. We compute among others the $\Delta m_{i j}^{2}$ and $\sin \theta_{i j}^{2}$ oscillation parameters for both normal $(\mathrm{NH})$ and inverted $(\mathrm{IH})$ mass hierarchies with numerical estimations that agree with advanced theoretical modeling in this matter; and which fit well with recent experimental measurements on neutrinos. Second, we use results on the perturbation of the scalar potential of the theory by higher dimensional operators suppressed by powers of the Planck scale $M_{\mathrm{Pl}}$; and on the effective field action approach in the flavon sector of FNMSSM to propose a scenario preventing the domain wall problem created by the spontaneous breaking of the full flavor group $G_{\mathrm{f}}$ in $F N M S S M$.

The building blocks of FNMSSM are given by the usual NMSSM ones supplemented by two other kinds of chiral superfields: $(\boldsymbol{\alpha})$ three right-handed neutrinos $N_{i}^{c}$ in order to have massive neutrinos, and $(\boldsymbol{\beta})$ three sets of flavon superfields $\Phi, \Omega, \chi$, with quantum numbers as in Tables I-III, allowing us to generate suitable neutrino masses and mixing as well as avoiding DWs. To build the chiral superpotential of the FNMSSM prototype, we extend the $S M$ gauge symmetry group by the global discrete flavor symmetry $\mathbb{A}_{4} \times \boldsymbol{Z}_{3}$. Notice that a supersymmetric model based on $\mathbb{A}_{4} \times \boldsymbol{Z}_{3}$ group was first proposed by Altarelli and Feruglio in [63] and predicted a mixing of

TABLE I. Gauge charges of lepton and Higgs superfields of the model.

\begin{tabular}{lrrrrrrrr}
\hline \hline Superfields & $L_{i}$ & $e^{c}$ & $\mu^{c}$ & $\tau^{c}$ & $N_{i}^{c}$ & $H_{u}$ & $H_{d}$ & $S$ \\
\hline$S U(2)_{L}$ & 2 & 1 & 1 & 1 & 1 & 2 & 2 & 1 \\
$U(1)_{Y}$ & -1 & 2 & 2 & 2 & 0 & 1 & -1 & 0 \\
\hline \hline
\end{tabular}


TABLE II. Lepton and right-handed neutrino superfields and their quantum numbers under $\mathbb{A}_{4} \times Z_{3}$.

\begin{tabular}{lccccc}
\hline \hline Superfields & $L_{i}$ & $e^{c}$ & $\mu^{c}$ & $\tau^{c}$ & $N_{i}^{c}$ \\
\hline $\mathbb{A}_{4}$ & $\mathbf{3}_{(-1,0)}$ & $1_{\left(1, \omega^{2}\right)}$ & $1_{(1, \omega)}$ & $1_{(1,1)}$ & $\mathbf{3}_{(-1,0)}$ \\
$\boldsymbol{Z}_{3}$ & $\mathbf{1}_{Q}$ & $\mathbf{1}_{Q}$ & $\mathbf{1}_{Q}$ & $\mathbf{1}_{Q}$ & $\mathbf{1}_{Q^{2}}$ \\
\hline \hline
\end{tabular}

TABLE III. Higgs and flavon superfields and their quantum numbers under $\mathbb{A}_{4} \times \boldsymbol{Z}_{3}$.

\begin{tabular}{lcccccc}
\hline \hline Superfields & $H_{u}$ & $H_{d}$ & $\Phi$ & $\Omega$ & $S$ & $\chi$ \\
\hline $\mathbb{A}_{4}$ & $\mathbf{1}_{(1,1)}$ & $\mathbf{1}_{(1, \omega)}$ & $\mathbf{3}_{(-1,0)}$ & $\mathbf{3}_{(-1,0)}$ & $\mathbf{1}_{\left(1, \omega^{2}\right)}$ & $\mathbf{1}_{(1,1)}$ \\
$\boldsymbol{Z}_{3}$ & $\mathbf{1}_{1}$ & $\mathbf{1}_{Q}$ & $\mathbf{1}_{1}$ & $\mathbf{1}_{Q^{2}}$ & $\mathbf{1}_{Q^{2}}$ & $\mathbf{1}_{Q^{2}}$ \\
\hline \hline
\end{tabular}

TBM type. Here, by using the same flavor group we work in the context of the NMSSM which is motivated by the several implications carried by its singlet superfield $S$ where besides its phenomenological advantages mentioned above it also contributes to the Higgs mass and to the neutrino masses. The non-Abelian alternating $\mathbb{A}_{4}$ group is introduced to explain the recent experimental data on neutrino oscillation experiments, while the $\boldsymbol{Z}_{3}$ symmetry is imposed to avoid the interchange between the flavon superfields $\Phi$ and $\Omega$ respectively appearing in the charged and chargeless lepton sectors. In the neutrino sector, the tiny neutrinos masses are generated through type I seesaw mechanism where besides the usual NMSSM singlet $S$, two flavons are required to achieve a neutrino mass matrix compatible with the TBM matrix. Notice that the contribution of the singlet $S$ is naturally small [64]—it must be at the SUSY scale (few TeVs) — compared to the flavon fields added in the lepton sector; thus, providing another motivation of working in the framework of the NMSSM. Accordingly, $S$ may be viewed as a small perturbation of the Majorana neutrino mass matrix - breaking the $\mu-\tau$ symmetry [65-67] — leading to a deviation of TBM; for a recent review on this manner of doing, see [68] and references therein. The mixing matrix in this scenario is obtained by rotating the TBM in the 1-3 plane, and thus leading to the so-called trimaximal mixing $\left(\mathrm{TM}_{2}\right)$ [44,46,69-73]. Next, we perform a numerical study concerning the neutrino mixing angles for both $\mathrm{NH}$ and $\mathrm{IH}$ where we find that they all fit the current experimental data. We have also studied the phenomenological consequences of the model where we obtained interesting predictions regarding the effective Majorana neutrino mass $m_{e e}$ measured in neutrinoless double beta decay experiments, the effective electron neutrino mass $m_{\nu_{e}}$ measured in tritium beta decay experiments and the Dirac CPV phase. In particular, the obtained ranges of $m_{e e}$ and $m_{\nu_{e}}$ may be tested in current and near future experiments, and which will first start to explore the regions corresponding to the inverted mass hierarchy.
As mentioned above, the NMSSM suffers from the DW problem coming from the spontaneous breaking of the discrete $\mathcal{Z}_{3}$ symmetry at the electroweak phase transition $[14,20,74]$. In our FNMSSM prototype, the DW problem arises from the spontaneous breaking of the $\mathbb{A}_{4} \times \boldsymbol{Z}_{3}$ flavor symmetry bearing in mind that the charge assignments under the extra $\boldsymbol{Z}_{3}$ discrete group is different from those in the usual NMSSM. In particular, two $\mathbb{A}_{4}$ breaking patterns are analysed: (i) the first one driven by an $\mathbb{A}_{4}$ triplet leads to the spontaneous breaking of $G_{\mathrm{f}}$ down to its subgroup $\mathbb{Z}_{3} \times$ $Z_{3}$ in the charged lepton sector, where the domain walls appear as the boundaries separating the degenerate vacua generated by this breaking. We find that these DWs are remarkably described by the Klein-four $\mathbb{V}_{4} \cong \mathbb{Z}_{2} \times \mathbb{Z}_{2}$ flavor subsymmetry group, and are formed around the inflationary scale; and thus, present no danger from the cosmological view. (ii) the second breaking is given by $G_{\mathrm{f}}$ to a subgroup $\mathbb{Z}_{2}$ in the neutrino sector, it is generated by the VEV of $\Omega$ and $S$ and creates domain walls that expand between the boundaries of degenerate vacua which are characterized by the $\mathbb{Z}_{2} \rtimes \mathbb{Z}_{3} \times Z_{3}$ subgroup of $G_{\mathrm{f}}$. The standard cosmology requires that these domain walls disappear at least before nucleosynthesis [75], and one of the first proposed solution suggests that, after breaking the degeneracy of the vacua, the true vacuum dominates [16]. Here, we show that breaking explicitly the $\mathbb{Z}_{2} \rtimes$ $\mathbb{Z}_{3} \times \boldsymbol{Z}_{3}$ subsymmetry of $G_{\mathrm{f}}$ at high energy by means of additional nonrenormalizable terms-Planck-suppressed operators-is capable to remove the degeneracy between the different domains. In addition, the theory at low energy will not undergo a remarkable change as long as these operators are suppressed by powers of the Planck scale.

This paper is organized as follows. In Sec. II, we present our FNMSSM proposal giving an extension of NMSSM with right-handed neutrinos and a global discrete $\mathbb{A}_{4} \times \boldsymbol{Z}_{3}$ flavor symmetry. Within this section we give the superfield properties describing the flavored NMSSM, then we describe the $\mathbb{A}_{4} \times \boldsymbol{Z}_{3}$ invariant Yukawa couplings in the charged lepton sector. In Sec. III, we study the neutrino masses and mixing as well as the deviation from TBM matrix, and we use the $3 \sigma$ values of the neutrino oscillation parameters to extract the allowed ranges of our model parameters. In Sec. IV, we perform a phenomenological study of our model and provide the predictions concerning the parameters $m_{e e}, m_{\nu_{e}}$ and the Dirac CPV phase $\delta_{C P}$ along with the Jarlskog parameter and its impact on the $\theta_{23}$ octant degeneracy. In Sec. V, we discuss the domain wall problem in our FNMSSM created via the spontaneous breaking of $G_{\mathrm{f}}$ and we provide a viable solution to this cosmological problem in the neutrino sector by adding a Planck-suppressed operator that breaks explicitly $G_{\mathrm{f}}$. In Sec. VI, we give our conclusion and then we give four appendices A, B, C and D. In Appendix A, we provide some useful details on the discrete $A_{4}$ group and its representations. In Appendix B, we determine the vacuum 
alignments of the $\mathbb{A}_{4}$ flavon triplets $\Phi$ and $\Omega$. In Appendix $\mathrm{C}$, we give some comments on additional nonrenormalizable operators that break explicitly the $\mathbb{A}_{4} \times Z_{3}$ group and we illustrate the contribution of one of these operators to the effective potential of the theory. In Appendix D, we describe the case where the breaking pattern in the neutrino sector is driven by the VEV of the NMSSM singlet $S$.

\section{BUILDING BLOCKS IN FLAVON EXTENDED NMSSM}

In this section, we introduce the flavon extended NMSSM that we propose to produce neutrino masses, their mixing and bypassing the domain wall problem created by spontaneous breaking of the flavor symmetry. To get straight to the point, we will mainly focus on leptons by first giving the chiral superfield content of the model; then describing the $\mathbb{A}_{4} \times \boldsymbol{Z}_{3}$ invariant Yukawa coupling in the charged lepton sector.

\section{A. Superfield content of the model}

In addition to the usual chiral and gauge superfields of the MSSM, our model contains extra chiral superfields carrying quantum numbers under the $\mathbb{A}_{4} \times \boldsymbol{Z}_{3}$ flavor symmetry group:

(i) the chiral singlet superfield $S$ of NMSSM that couples to the usual Higgs doublets $H_{u}$ and $H_{d}$. This complex superfield carries a negative unit charge under $\boldsymbol{Z}_{3}$; and carry nontrivial quantum charge under $\mathbb{A}_{4}$.

(ii) three right-handed neutrinos $N_{i=1,2,3}^{c}$ needed to generate tiny masses for neutrinos through type I seesaw mechanism. They carry a negative charge under $\boldsymbol{Z}_{3}$ exactly like $S$; but form altogether an irreducible triplet under $\mathbb{A}_{4}$.

(iii) three flavon superfields $\{\Phi, \Omega, \chi\}$ which are gauge singlets but carry non-trivial quantum numbers under $\mathbb{A}_{4} \times \boldsymbol{Z}_{3}$ symmetry.

The $\mathbb{A}_{4} \times \boldsymbol{Z}_{3}$ representations hosting these chiral superfields and the role they play in the construction are described below.

\section{Quantum charges}

The gauge quantum numbers under $S U(2)_{L} \times U(1)_{Y}$ of the lepton and the Higgs superfields of the NMSSM as well as the right-handed neutrinos $N_{i}^{c}$ are as shown in Table I. The lepton doublets of the three generations $L_{i}=$ $\left(L_{e}, L_{\mu}, L_{\mu}\right)$ and the three right-handed neutrinos $N_{i}^{c}=$ $\left(\nu_{e}^{c}, \nu_{\mu}^{c}, \nu_{\tau}^{c}\right)$ are assigned to irreducible triplets $\mathbf{3}_{(-1,0)}$ of the discrete group $\mathbb{A}_{4}$. The three right-handed leptons $E_{i}^{c}=$ $\left(e^{c}, \mu^{c}, \tau^{c}\right)$ sit however in the three different $\mathbb{A}_{4}$ singlets $\mathbf{1}_{\left(1, \omega^{2}\right)}, \mathbf{1}_{(1, \omega)}, \mathbf{1}_{(1,1)}$ where $\omega=e^{i \frac{i \pi}{3}}$ while $(1,1),(1, \omega)$ and $\left(1, \omega^{2}\right)$ denote the group characters used to discriminate the one- dimensional representations of the $A_{4}$ group. Regarding the Higgs superfields of the NMSSM namely the usual three $\left(H_{u}, H_{d}, S\right)$ are hosted by $\left(\mathbf{1}_{(1,1)}, \mathbf{1}_{(1, \omega)}\right.$, $\left.\mathbf{1}_{\left(1, \omega^{2}\right)}\right)$; and carry charges of the extra discrete $\boldsymbol{Z}_{3}$. In addition to the superfields in Table I, the three flavon superfields $\{\Phi, \Omega, \chi\}$ carry nontrivial quantum numbers under $\mathbb{A}_{4}$ symmetry as shown in Table III. The flavon $\Phi$, transforming as $\mathbf{3}_{(-1,0)}$ under $\mathbb{A}_{4}$, is added in the charged lepton sector; while the $\Omega$ and $\chi$ are both added in the neutrino sector. We will show later that the flavon $A_{4}$ - triplet $\Omega$ with the $\mathbb{A}_{4}$ - singlet superfield $\chi$ are required to produce a neutrino mass matrix compatible with the tribimaximal mixing; while $S$, hosted by the $\mathbf{1}_{\left(1, \omega^{2}\right)}$ of the $\mathrm{A}_{4}$ symmetry is needed for the deviation from TBM matrix. Moreover, to obtain the right vacuum alignment of the $\mathbb{A}_{4}$ triplets $\Phi$ and $\Omega$ that lead to the desired masses and mixing, we need to avoid the communication between the charged lepton and neutrino sectors. This is achieved by introducing the $\boldsymbol{Z}_{3}$ discrete symmetry under which the flavons triplets $\Phi$ and $\Omega$ act differently. The $\boldsymbol{Z}_{3}$ quantum numbers for all the superfields are as given in Tables II and III with $Q=e^{i \frac{2 \pi}{3}}$. To fix ideas on some useful features of the $\mathbb{A}_{4} \times \boldsymbol{Z}_{3}$ flavor symmetry of the proposed model, recall that the subgroup $\mathbb{A}_{4}$ has four irreducible representations $\mathbf{1}, \mathbf{1}^{\prime}, \mathbf{1}^{\prime \prime}$ and $\mathbf{3}$ used to host the chiral superfield of the model; while the $\boldsymbol{Z}_{3}$ subsymmetry has three irreducible representations $\tilde{\mathbf{1}}, \tilde{\mathbf{1}}^{\prime}, \tilde{\mathbf{1}}^{\prime \prime}$. The properties of these representations will play an important role in this study; for this reason, and also for the study of the breaking of $\mathbb{A}_{4}$ down to its $\mathbb{Z}_{3}$ and $\mathbb{Z}_{2}$ subgroups, we thought it is useful to recall briefly some useful properties on the $\mathbb{A}_{4}$ group to better illustrate the construction.

\section{Flavor symmetry $G_{\mathrm{f}}=\mathbb{A}_{4} \times Z_{3}$}

The alternating $\mathbb{A}_{4}$ subsymmetry of $G_{\mathrm{f}}$ is a non-Abelian discrete group living inside of the well known permutation group $\mathbb{S}_{4}$. It is generated by two noncommuting operators $\mathcal{S}$ and $\mathcal{T}(\mathcal{S} \mathcal{T} \neq \mathcal{T} \mathcal{S})$ with the property $\mathcal{S}^{2}=\mathcal{T}^{3}=I$, these $\mathcal{S}$ and $\mathcal{T}$ cannot be diagonalized simultaneously; and we will use later the basis where $\mathcal{T}$ is represented by a diagonal matrix [63]. The order of the $\mathbb{A}_{4}$ group is 12 ; it is related to the dimensions of its four irreducible representations through the character relation

$$
\mathbb{A}_{4}: 12=(1)^{2}+\left(1^{\prime}\right)^{2}+\left(1^{\prime \prime}\right)^{2}+(3)^{2}
$$

Throughout this work, we will denote these four irreducible representations by their basis characters as $\mathbf{3}_{(-1,0)}, \mathbf{1}_{(1,1)}$, $\mathbf{1}_{(1, \omega)}$ and $\mathbf{1}_{\left(1, \omega^{2}\right)}$ with $\omega=\exp (2 i \pi / 3)$; this notation gives a tricky manner to distinguish the three one- dimensional representations by the characters of the generators of $\mathbb{A}_{4}$. Because of the property $\omega^{2}=\bar{\omega}$, we can also denote the one- dimensional representations as $\mathbf{1}_{(1,1)}, \mathbf{1}_{(1, \omega)}, \mathbf{1}_{(1, \bar{\omega})}$; for other aspects, see Appendix A and Refs. [76,77]. 
The $\mathbb{A}_{4}$ has ten subgroups that we want to describe briefly; they are needed in Sec. IV when we study the breaking of $\mathbb{A}_{4}$. All the proper subgroups of $\mathbb{A}_{4}$ are Abelian and so have one- dimensional irreducible representations that can be determined by using the analogue of the character formula (2.1). In addition to the set $\mathbb{A}_{4}$ itself and to the identity $I_{i d}$, the subgroups of $\mathbb{A}_{4}$ are as follows: (a) the Klein-four group $\mathbb{V}_{4}$ with non trivial elements given by double transpositions $(i j)(k l)$ as reported hereinbelow

$$
\mathbb{V}_{4}=\left\{I_{i d},(12)(34),(13)(24),(14)(23)\right\}
$$

where $\{1,2,3,4\}$ are numbers indexing some four given objects $\left\{X_{1}, X_{2}, X_{3}, X_{4}\right\}$. In our case, the $\Phi_{i}, \Omega_{i}$ and $\chi$ flavon chiral superfields are given by a subsets of $X_{i}$ 's or also by linear combinations of them. (b) four kinds of cyclic groups $\mathbb{Z}_{3}$, one of them is given by the set $\left\{I_{i d},(123),(132)\right\}$ where the fourth point $X_{4}$ is fixed; a second example of a $\mathbb{Z}_{3}$ subgroup is given by $\left\{I_{i d},(124),(142)\right\}$ where now the point $X_{3}$ which is fixed. (c) three cyclic groups $\mathbb{Z}_{2}$ generated by double transpositions; they are subgroups of $\mathbb{V}_{4}$; one of these three $\mathbb{Z}_{2}$ 's is given by $\left\{I_{i d},(12)(34)\right\}$; a second one is given by $\left\{I_{i d},(13)(24)\right\}$. The multiplicities of these subgroups are as in the following Table

\begin{tabular}{|l|l|l|l|l|l|}
\hline subgroups & $\mathbb{A}_{4}$ & $\mathbb{V}_{4}$ & $\mathbb{Z}_{3}$ & $\mathbb{Z}_{2}$ & $I_{i d}$ \\
\hline order & 12 & 4 & 3 & 2 & 1 \\
\hline multiplicity & 1 & 1 & 4 & 3 & 1 \\
\hline
\end{tabular}

The $\boldsymbol{Z}_{3}$ subsymmetry of $G_{\mathrm{f}}$ is an Abelian discrete group which has not to be confused with $\mathbb{Z}_{3}$ which is a subgroup of $\mathbb{A}_{4}$; it has three different one- dimensional representations $\tilde{\mathbf{1}}, \tilde{\mathbf{1}}^{\prime}, \tilde{\mathbf{1}}^{\prime \prime}$ that can be denoted as well like $\mathbf{1}_{1}, \mathbf{1}_{Q}$, and $\mathbf{1}_{Q^{2}}$ with $Q=e^{i \frac{2 \pi}{3}}$.

To summarize, the flavor symmetry $G_{\mathrm{f}}=\mathbb{A}_{4} \times \boldsymbol{Z}_{3}$ has three generators $\mathcal{S}, \mathcal{T}$, and $\tilde{\mathcal{T}}$ and 12 irreducible representations namely

$$
\begin{array}{lccc}
\mathbf{1}_{(1,1,1)}, & \mathbf{1}_{(1, \omega, 1)}, & \mathbf{1}_{(1, \bar{\omega}, 1)} & \mathbf{3}_{(-1,0,1)} \\
\mathbf{1}_{(1,1, Q)}, & \mathbf{1}_{(1, \omega, Q)}, & \mathbf{1}_{(1, \bar{\omega}, Q)} & \mathbf{3}_{(-1,0, Q)} \\
\mathbf{1}_{(1,1, \bar{Q})}, & \mathbf{1}_{(1, \omega, \bar{Q})}, & \mathbf{1}_{(1, \bar{\omega}, \bar{Q})} & \mathbf{3}_{(-1,0, \bar{Q})}
\end{array}
$$

where the indices refer to the characters of the generators, the two first entries for the $\mathcal{S}, \mathcal{T}$ of alternating $\mathbb{A}_{4}$ and the third for the $\tilde{\mathcal{T}}$ of $\boldsymbol{Z}_{3}$.

\section{B. Charged lepton sector}

With the extra $\mathbb{A}_{4} \times \boldsymbol{Z}_{3}$ quantum numbers; we have a powerful tool to suppress undesired superfield couplings in building the general lepton mass matrix. Following [63], the usual Yukawa couplings, giving the masses $\left(m_{e}, m_{\mu}, m_{\tau}\right)$ of the charged leptons, are given by the $W_{\text {lep }^{+}}^{0}=$ $\sum_{i, j} y_{0}^{i j} H_{d} E_{i}^{c} L_{j}$ tri-superfield couplings which in general are not invariant under the flavor symmetry. To make the charged leptons superpotential invariant under the full discrete $\mathbb{A}_{4} \times Z_{3}$ symmetry group, we promote the Yukawa coupling constants $y_{0}^{i j}$ to be flavon $\Phi$ - dependent like $y_{0}^{i j} \rightarrow y_{0}^{i j k} \frac{\Phi_{k}}{\Lambda}$ where $\Lambda$ denotes a cutoff scale of the $G_{\mathrm{f}}$ symmetry of GUT scale order. Thus, the desired $W_{\text {lep }}{ }^{+}$chiral superpotential for the charged leptons is flavon dependant $\left.W_{\text {lep }}+\Phi\right]$ and is given by a four- order operator generated by the monomials $H_{d} E_{i}^{c} L_{j} \Phi_{k}$. However, because $L_{j}$ and $\Phi_{k}$ transform into nontrivial representations of $G_{\mathrm{f}}$, these four order monomials carry non trivial quantum charges of the discrete flavor symmetry. In the $\mathbb{A}_{4} \times \boldsymbol{Z}_{3}$ representation language, the $H_{d} E_{i}^{c} L_{j} \Phi_{k}$ transform in the $\mathbb{A}_{4}$ - tensor product representation $\mathbf{1}_{\left(1, \bar{\omega}^{i-1}\right)} \otimes\left[\mathbf{3}_{(-1,0)} \otimes \mathbf{3}_{(-1,0)}\right]$ as it can be checked from Tables II and III. From this tensor product structure, we learn that the $3^{3}=27$ possible monomials $H_{d} E_{i}^{c} L_{j} \Phi_{k}$ are manifestly $\boldsymbol{Z}_{3}$ - invariant $\left(Q^{3}=1\right)$; while invariance under the non-Abelian $\mathbb{A}_{4}$ requires restricting the sum to the particular trace

$$
\left.\operatorname{Tr}_{\mathrm{A}_{4}}\left(\frac{y^{i j k}}{\Lambda} E_{i}^{c} L_{j} \Phi_{k}\right) \equiv\left(\frac{y^{i j k}}{\Lambda} E_{i}^{c} L_{j} \Phi_{k}\right)\right|_{\mathbf{1}_{(1, \bar{\omega})}}
$$

This trace can be obtained by first using the group representation notations $L \otimes \Phi \equiv \mathbf{3}_{(-1,0)} \otimes \mathbf{3}_{(-1,0)}$ showing that $L_{j} \Phi_{k}$, with $L_{j}=\left(L_{e}, L_{\mu}, L_{\tau}\right)$ and $\Phi_{k}=\left(\Phi_{1}, \Phi_{2}, \Phi_{3}\right)$, has nine components carrying in general different $\mathbb{A}_{4}$ - quantum numbers. By using results from on the reducibility property of tensors product of $\mathbb{A}_{4}$ - representations; in particular $\mathbf{3}_{(-1,0)} \otimes \mathbf{3}_{(-1,0)}=\mathbf{9}_{(1,0)}$ with

$\mathbf{9}_{(1,0)}=\mathbf{1}_{(1,1)} \oplus \mathbf{1}_{(1, \omega)} \oplus \mathbf{1}_{(1, \bar{\omega})} \oplus \mathbf{3}_{(-1,0)} \oplus \mathbf{3}_{(-1,0)}$

we learn that the relevant terms in the charged lepton superpotential $W_{\text {lep }^{+}}$are given, in the $\mathbb{A}_{4}$ basis where the generator $\mathcal{T}$ is diagonal $\left[(\mathcal{T} . \Phi)_{r}=\omega^{r-1} \Phi_{r}\right.$ and $(\mathcal{T} . L)_{r}=\omega^{r-1} L_{r}$ ], by the following one dimensional $\mathrm{A}_{4}$ - representations

$$
\begin{aligned}
& \left.L \otimes \Phi\right|_{\mathbf{1}_{(1,1)}}=L_{e} \Phi_{1}+L_{\tau} \Phi_{2}+L_{\mu} \Phi_{3} \\
& \left.L \otimes \Phi\right|_{\mathbf{1}_{(1, \omega)}}=L_{\mu} \Phi_{1}+L_{e} \Phi_{2}+L_{\tau} \Phi_{3} \\
& \left.L \otimes \Phi\right|_{\mathbf{1}_{(1, \bar{\omega})}}=L_{\tau} \Phi_{1}+L_{\mu} \Phi_{2}+L_{e} \Phi_{3}
\end{aligned}
$$

For the explicit expressions of the other irreducible representations; see Eq. (A3) of Appendix A. Notice that the above restrictions to the three one- dimensional $\left.L \otimes \Phi\right|_{\mathbf{1}_{\left(1, \omega^{r}\right)}}$ of the $\mathbb{A}_{4}$ symmetry are because of the quantum numbers of the right handed leptons $E_{i}^{c}=\left(e^{c}, \mu^{c}, \tau^{c}\right)$ which transform under $\mathbb{A}_{4}$ into three different one dimensional 
representations like $E_{i}^{c} \sim \mathbf{1}_{\left(1, \bar{\omega}^{i}\right)}$. Therefore, the desired $\mathrm{A}_{4}$ - invariant quantity is given by

$\operatorname{Tr}_{\mathbb{A}_{4}}\left(\frac{y^{i j k}}{\Lambda} E_{i}^{c} L_{j} \Phi_{k}\right) \sim \sum_{i=1}^{3}\left(\mathbf{1}_{\left(1, \omega^{-i}\right)} \times\left.(L \otimes \Phi)\right|_{\mathbf{1}_{\left(1, \omega^{i-1}\right)}}\right)$

leading to the following quartic $\mathbb{A}_{4} \times Z_{3}$ invariant superpotential

$$
W_{\mathrm{lep}^{+}}=\sum_{i=1}^{3} \frac{y_{i}}{\Lambda} H_{d}\left(\left.L \otimes \Phi\right|_{\mathbf{1}_{\left(1, \omega^{i-1}\right)}}\right) E_{i}^{c}
$$

Taking the VEV of $\mathbb{A}_{4}$ - triplet $\Phi$ like $\langle\Phi\rangle=\left(v_{\Phi}, 0,0\right)$ and the VEV of the Higgs $H_{d}$ as usual- $\left\langle H_{d}\right\rangle=v_{d^{-}}$; then substituting these expressions back into the superpotential (2.9), we obtain the desired supersymmetric Yukawa couplings

$$
\begin{aligned}
W_{\mathrm{lep}^{+}}= & \frac{y_{e} v_{\Phi}}{\Lambda} H_{d} e^{c} L_{e}+\frac{y_{\mu} v_{\Phi}}{\Lambda} H_{d} \mu^{c} L_{\mu} \\
& +\frac{y_{\tau} v_{\Phi}}{\Lambda} H_{d} \tau^{c} L_{\tau}
\end{aligned}
$$

$$
U_{\mathrm{PMNS}}=\left(\begin{array}{c}
c_{12} c_{13} \\
-c_{23} s_{12}-c_{12} s_{13} s_{23} e^{i \delta} \\
s_{12} s_{23}-c_{12} c_{23} s_{13} e^{i \delta}
\end{array}\right.
$$

where the short hands $c_{i j} \equiv \cos \theta_{i j}$ and $s_{i j} \equiv \sin \theta_{i j}$ refer to the mixing angles; $\delta$ refers to the Dirac $C P$ phase and $\mathcal{U}_{m}=\operatorname{diag}\left(1, e^{\frac{i}{2} \alpha_{21}}, e^{\frac{i}{2} \alpha_{31}}\right)$ encodes the Majorana-type $C P$ violating phases $\alpha_{21}$ and $\alpha_{31}$.

\section{A. Trimaximal mixing as a deviation of TBM matrix}

By adding the three right-handed neutrinos $N_{i}^{c}=$ $\left(\nu_{e}^{c}, \nu_{\mu}^{c}, \nu_{\tau}^{c}\right)$, the light neutrino masses are generated via type I seesaw mechanism formula $m_{\nu}=m_{D}^{T} M_{R}^{-1} m_{D}$ with $M_{R}$ the right-handed Majorana neutrinos mass matrix. The Dirac mass matrix $m_{D}$ emerges from the Yukawa couplings of the left and right-handed neutrinos with the Higgs superfield $H_{u}$. The relevant chiral superpotential $W_{D}$ respecting gauge and flavor symmetries of the model is as follows

$$
W_{D}=\operatorname{Tr}_{\mathbb{A}_{4}}\left(Y^{i j} L_{i} N_{j}^{c} H_{u}\right)
$$

where $L_{i}=\left(L_{e}, L_{\mu}, L_{\tau}\right), Y^{i j}$ are Yukawa coupling constants and where $\operatorname{Tr}_{\mathbb{A}_{4}}[*]$ refers to the restriction of the sum $\sum_{i, j} Y^{i j} L_{i} N_{j}^{c}$ to the $\mathbb{A}_{4}$ - invariant part. Following the same leading to a diagonal charged lepton mass matrix $M_{\mathrm{lep}^{+}}=$ $\operatorname{diag}\left(m_{e}, m_{\mu}, m_{\tau}\right)$ with mass eigenvalues induced by the product of two VEVs as follows

$m_{e}=y_{e} \frac{v_{d} v_{\Phi}}{\Lambda}, \quad m_{\mu}=y_{\mu} \frac{v_{d} v_{\Phi}}{\Lambda}, \quad m_{\tau}=y_{\tau} \frac{v_{d} v_{\Phi}}{\Lambda}$

By using the experimental value of the tau mass as given by the Particle Data Group [78], namely $m_{\tau}=1776.86 \mathrm{MeV}$, and by assuming that $y_{\tau} v_{d} \lesssim 246 \mathrm{GeV}$, we obtain a lower bound on the ratio between the flavon VEV $v_{\Phi}$ and the cutoff scale $\Lambda$ given by

$$
\frac{v_{\Phi}}{\Lambda}>0.007
$$

This constraint on $v_{\Phi}$ will be used later on when studying domain walls in the charged lepton sector.

\section{NEUTRINO MASSES AND TRIMAXIMAL MIXING}

In this section, we study the neutrino masses and mixing in the framework of trimaximal scheme in order to reconcile with the experimental values of the reactor and atmospheric mixing angles. We use the PDG parametrization for the mixing matrix $U_{\mathrm{PMNS}}$ given by [78]

$\left.\begin{array}{cc}c_{13} s_{12} & s_{13} e^{-i \delta} \\ c_{12} c_{23}-s_{12} s_{13} s_{23} e^{i \delta} & c_{13} s_{23} \\ -c_{12} s_{23}-c_{23} s_{12} s_{13} e^{i \delta} & c_{13} c_{23}\end{array}\right) \cdot \mathcal{U}_{m}$

analysis used in the charged lepton sector, $\mathbb{A}_{4}$-invariant $W_{D}$ is then given by $\left.\left(H_{u} \otimes L \otimes N^{c}\right)\right|_{\mathbf{1}_{(1,1)}}$; but because $H_{u} \sim \mathbf{1}_{(1,1)}$, this irreducible component reduces to $H_{u}$ times $\left.\left(L \otimes N^{c}\right)\right|_{\mathbf{1}_{(1,1)}}$ reading explicitly as

$$
W_{D}=Y_{0} H_{u}\left(L_{1} N_{1}^{c}+L_{2} N_{3}^{c}+L_{3} N_{2}^{c}\right)
$$

Giving to the neutral component of the $H_{u}$ Higgs doublet its VEV $v_{u}$, we get the Dirac mass matrix of neutrinos

$$
m_{D}=Y_{0} v_{u}\left(\begin{array}{ccc}
1 & 0 & 0 \\
0 & 0 & 1 \\
0 & 1 & 0
\end{array}\right)
$$

On the other hand, the superpotential $W_{R}$ of the righthanded Majorana neutrinos respecting gauge invariance, the $\mathbb{A}_{4} \times \boldsymbol{Z}_{3}$ flavor symmetry and leading to trimaximal mixing, contains three tri-superfield couplings as in Eq. (3.5) given below; the first one involves the chiral superfield $\chi$ which is an $\mathbb{A}_{4}$ - singlet, the second one involves an $\mathbb{A}_{4}$ - flavon triplet $\Omega=\left(\Omega_{1}, \Omega_{2}, \Omega_{3}\right)$ and the 
last one involves the NMSSM singlet $S$ which transforms as a nontrivial singlet under $\mathbb{A}_{4}$. The superpotential having a quadratic dependence into the right-handed neutrinos superfields-i.e.: $\mathrm{W}_{R}$ of the form $\sum \lambda_{i} N^{c} \Phi_{i} N^{c}$-is expressed at the renormalizable level as ${ }^{1}$

$$
\begin{aligned}
W_{R}= & \lambda \operatorname{Tr}_{\mathbb{A}_{4}}\left(\chi N^{c} N^{c}\right)+\lambda^{\prime} \operatorname{Tr}_{\mathbb{A}_{4}}\left(\Omega N^{c} N^{c}\right) \\
& +\lambda^{\prime \prime} \operatorname{Tr}_{\mathbb{A}_{4}}\left(S N^{c} N^{c}\right)
\end{aligned}
$$

where $\lambda, \lambda^{\prime}$. and $\lambda^{\prime \prime}$ are Yukawa coupling constants. The first tri-superfield coupling $\chi N^{c} N^{c}$ transforms under the $\mathbb{A}_{4}$ symmetry as $1_{(1,1)} \otimes 3_{(-1,0)} \otimes 3_{(-1,0)}$, the second coupling $\Omega N^{c} N^{c}$ transforms like $3_{(-1,0)} \otimes 3_{(-1,0)} \otimes 3_{(-1,0)}$; while the third coupling $S N^{c} N^{c}$ transforms as $1_{\left(1, \omega^{2}\right)} \otimes 3_{(-1,0)} \otimes 3_{(-1,0)}$. Hence, by using the Clebsch-Gordan decomposition of $\mathbb{A}_{4}$ (A3), the superpotential $W_{R}$ develops into

$$
\begin{aligned}
W_{R}= & \lambda \chi\left(\nu_{e}^{c} \nu_{e}^{c}+\nu_{\mu}^{c} \nu_{\tau}^{c}+\nu_{\tau}^{c} \nu_{\mu}^{c}\right)+\lambda^{\prime \prime} S\left(\nu_{e}^{c} \nu_{\mu}^{c}+\nu_{\mu}^{c} \nu_{e}^{c}+\nu_{\tau}^{c} \nu_{\tau}^{c}\right) \\
& +\frac{2 \lambda^{\prime}}{3}\left(\left[\left(\nu_{e}^{c} \nu_{e}^{c}-\nu_{\mu}^{c} \nu_{\tau}^{c}\right) \Omega_{1}+\left(\nu_{\tau}^{c} \nu_{\tau}^{c}-\nu_{e}^{c} \nu_{\mu}^{c}\right) \Omega_{2}\right.\right. \\
& \left.+\left(\nu_{\mu}^{c} \nu_{\mu}^{c}-\nu_{e}^{c} \nu_{\tau}^{c}\right) \Omega_{3}\right]
\end{aligned}
$$

By taking the flavon VEVs as $\langle\chi\rangle=v_{\chi},\langle\Omega\rangle=\left(v_{\Omega}, v_{\Omega}, v_{\Omega}\right)$ and the NMSSM singlet as $\langle S\rangle=v_{S}$, as well as adopting the following notations: $a=2 \lambda v_{\chi}, b=2 \lambda^{\prime} v_{\Omega}$ and $\epsilon=2 \lambda^{\prime \prime} v_{S}$ the parameters $a, b$ and $\epsilon$ have a dimension of mass and at least one of them should be complex as it will be shown below - the right-handed Majorana neutrino mass matrix $M_{R}$ is given by

$$
M_{R}=\left(\begin{array}{ccc}
a+\frac{2 b}{3} & -\frac{b}{3}+\epsilon & -\frac{b}{3} \\
-\frac{b}{3}+\epsilon & \frac{2 b}{3} & a-\frac{b}{3} \\
-\frac{b}{3} & a-\frac{b}{3} & \frac{2 b}{3}+\epsilon
\end{array}\right)
$$

In the limit $\epsilon \rightarrow 0$, this matrix is diagonalized by the TBM mixing matrix, given in Eq. (1.1). Notice by the way that the form of $M_{R}(\epsilon \rightarrow 0)$ was produced before with at least two flavons fields $[63,79,80]$; the novelty here is that we used the NMSSM singlet superfield $S$ to deviate from the TBM instead of a flavon field. It is well known that in this limit, the matrix respects the $\mu-\tau$ symmetry $^{2}$ with the prediction of zero reactor angle and maximal atmospheric angle.

\footnotetext{
${ }^{1}$ If dropping the condition of a superpotential quadratic in $N^{c}$, that is releasing the form $W_{R} \sim \lambda_{i} N^{c} \Phi_{i} N^{c}$, the superpotential may include other possible terms like $\lambda_{i j} N^{c} \Phi_{i} \Phi_{j}$. A possible manner to eliminate these undesirable couplings is to impose additional discrete symmetries. At the level of Eq. (3.5), linear and cubic $N^{c}$ - terms in $W_{R}$ may be eliminated by an extra $\mathcal{Z}_{2}: N^{c} \rightarrow-N^{c}$. In this case, the other matter superfields of the model $L$ and $E^{c}$ have to carry the same $\mathcal{Z}_{2}$ charge as $N^{c}$ while the scalar superfields carry a trivial $\mathcal{Z}_{2}$ charge.

${ }^{2}$ This symmetry is based on the invariance of the neutrino mass terms under the interchange of $\nu_{\mu}$ and $\nu_{\tau}$ [67].
}

Currently, it is a well-established experimental fact that the value of the reactor angle is different from zero; $\theta_{13} \neq 0$. On the other hand, the NOvA experiment has disfavored recently the maximal atmospheric angle; however, the significance of this indication, while at the $2.6 \sigma$ level in [81], was reduced to less than $1 \sigma$ in [82] which means that the maximal value of $\theta_{23}$ remain possible. Therefore, in order to produce the experimental values of these angles, we need to go beyond the TBM framework. Thus, by using the NMSSM singlet superfield $S$ (which plays the role of the deviation from TBM), a coupling $S N^{c} N^{c}$ in the Majorana superpotential (3.6) breaks the $\mu-\tau$ symmetry in a way that only the $\left(M_{R}\right)_{12},\left(M_{R}\right)_{21}$ and $\left(M_{R}\right)_{33}$ entries of the Majorana neutrino mass matrix are affected; see Eq. (3.7). The diagonalization of this symmetric matrix can be obtained by performing a similarity transformation like $M_{R}^{\prime}=\mathcal{P}^{-1} M_{R} \mathcal{P}$ with eigenvalues

$$
\begin{aligned}
& M_{1}^{\prime}=b+\sqrt{a^{2}-a \epsilon+\epsilon^{2}}, \quad M_{2}^{\prime}=a+\epsilon, \\
& M_{3}^{\prime}=b-\sqrt{a^{2}-a \epsilon+\epsilon^{2}}
\end{aligned}
$$

and

$$
\mathcal{P}=\left(\begin{array}{ccc}
\gamma_{1}^{+} & 1 & \gamma_{1}^{-} \\
\gamma_{2}^{+} & 1 & \gamma_{2}^{-} \\
1 & 1 & 1
\end{array}\right)
$$

with

$$
\begin{aligned}
& \gamma_{1}^{\mp}=\frac{(3 a+b) \epsilon-3 \epsilon^{2} \mp(3 \epsilon-2 b) \sqrt{a^{2}-a \epsilon+\epsilon^{2}}-2 a b}{a b-2 b \epsilon+3 \epsilon^{2}+(3 \epsilon-b) \sqrt{a^{2}-a \epsilon+\epsilon^{2}}} \\
& \gamma_{2}^{\mp}=\frac{(b-3 a) \epsilon \mp b \sqrt{a^{2}-a \epsilon+\epsilon^{2}}+a b}{a b-2 b \epsilon+3 \epsilon^{2}+(3 \epsilon-b) \sqrt{a^{2}-a \epsilon+\epsilon^{2}}} .
\end{aligned}
$$

These relations are nonlinear in the deviation parameter $\epsilon$; they may be treated perturbatively up to order $\mathcal{O}\left(\epsilon^{2}\right)$ by following the same method as done in [76]. For example, the linearization of the $b \pm \sqrt{a^{2}-a \epsilon+\epsilon^{2}}$ eigenvalues gives $b \pm a \mp \frac{\epsilon}{2}+O\left(\frac{\epsilon^{2}}{a}\right)$; and then

$M_{1}^{\prime} \simeq b+a-\frac{\epsilon}{2}, \quad M_{2}^{\prime}=a+\epsilon, \quad M_{3}^{\prime} \simeq b-a+\frac{\epsilon}{2}$

Nevertheless, to deal with (3.7) we follow the procedure used in [46] instead of the above linearization. The method relies on using the trimaximal mixing matrix $U_{\mathrm{TM}_{2}}=U_{\mathrm{TBM}} U_{R}$ obtained by multiplying $U_{\mathrm{TBM}}$ by a matrix $U_{R}$ from the right while leaving one of the columns in $U_{\mathrm{TBM}}$ unaffected. Here, $U_{R}$ stands for a "complexified rotation" matrix. In the (1-3) plane, we have 


$$
U_{R}=\left(\begin{array}{ccc}
\cos \theta & 0 & \sin \theta e^{-i \sigma} \\
0 & 1 & 0 \\
-\sin \theta e^{i \sigma} & 0 & \cos \theta
\end{array}\right)
$$

where the parameter $\theta$ parameterizes the deviation from tribimaximal mixing and $\sigma$ is an arbitrary phase that will be related to the Dirac $C P$ phase later. This complex rotation matrix $U_{R}$ has the properties $U_{R}^{\dagger} U_{R}=I$ and $\operatorname{det} U_{R}=1$; it preserves the second column of the TBM matrix and leads to

$$
U_{\mathrm{TM}_{2}}=\left(\begin{array}{ccc}
\sqrt{\frac{2}{3}} \cos \theta & \frac{1}{\sqrt{3}} & \sqrt{\frac{2}{3}} \sin \theta e^{-i \sigma} \\
-\frac{\cos \theta}{\sqrt{6}}-\frac{\sin \theta}{\sqrt{2}} e^{i \sigma} & \frac{1}{\sqrt{3}} & \frac{\cos \theta}{\sqrt{2}}-\frac{\sin \theta}{\sqrt{6}} e^{-i \sigma} \\
-\frac{\cos \theta}{\sqrt{6}}+\frac{\sin \theta}{\sqrt{2}} e^{i \sigma} & \frac{1}{\sqrt{3}} & -\frac{\cos \theta}{\sqrt{2}}-\frac{\sin \theta}{\sqrt{6}} e^{-i \sigma}
\end{array}\right)
$$

$$
\tan 2 \theta=\frac{-2 \sqrt{3}\left(2 k_{a \epsilon} \cos \phi_{a \epsilon}-1\right) \cos \sigma}{4\left(2 k_{a \epsilon} \cos \phi_{a \epsilon}-1\right)^{2} \cos ^{2} \sigma+\left(4 k_{a \epsilon} \cos \sigma \sin \phi_{a \epsilon}+4 k_{b \epsilon} \sin \sigma\right)^{2}}, \quad \tan \sigma=-k_{a b} \sin \phi_{a \epsilon} .
$$

For similar expressions to the above relations; see [83]. Furthermore, the exact eigenvalues of the mass matrix $M_{R}$ are given by $b \pm \sqrt{a^{2}-a \epsilon+\epsilon^{2}}$ and $a+\epsilon$; by assuming $\epsilon / a \ll 1$, they can be expanded in a power series in the reduced parameter $\epsilon / a$; at the leading order, we have:

$$
\begin{aligned}
& M_{1}=a+b-\frac{\epsilon}{2}+O\left(\frac{\epsilon^{2}}{a}\right), \quad M_{2}=a+\epsilon, \\
& M_{3}=b-a+\frac{\epsilon}{2}+O\left(\frac{\epsilon^{2}}{a}\right) .
\end{aligned}
$$

Now we turn to calculate the light neutrino masses by applying the type-I seesaw formula $m_{\nu}=m_{D}^{T} M_{R}^{-1} m_{D}$. Since $M_{R}$ is diagonalized by $U_{\mathrm{TM}_{2}}$ given in Eq. (3.13), the inverse Majorana neutrino mass is now given by

$$
M_{R}^{-1}=U_{\mathrm{TM}_{2}}^{*}\left[\operatorname{diag}\left(M_{1}, M_{2}, M_{3}\right)\right]^{-1}\left(U_{\mathrm{TM}_{2}}^{*}\right)^{T}
$$

Moreover, due to the form of the Dirac mass matrix (3.4), the diagonalization of $m_{\nu}$ remains of trimaximal form. ${ }^{3}$ Thus, the light neutrino masses are given by $\left(\tilde{U}_{\mathrm{TM}_{2}}\right)^{T} m_{\nu} \tilde{U}_{\mathrm{TM}_{2}}=$ $\operatorname{diag}\left(m_{1}, m_{2}, m_{3}\right)$ where $\tilde{U}_{\mathrm{TM}_{2}}=\frac{m_{D}}{Y_{0} v_{u}} U_{\mathrm{TM}_{2}}$,

\footnotetext{
${ }^{3}$ Notice that in general the light neutrino masses are given by $\tilde{U}_{\mathrm{TM}_{2}} m_{\nu}\left(\tilde{U}_{\mathrm{TM}_{2}}^{T}\right)=\operatorname{diag}\left(\hat{m}_{1}, \hat{m}_{2}, \hat{m}_{3}\right)$ where $\hat{m}_{j}=m_{j} \times e^{i \eta_{j}}$ are complex eigenvalues; the $m_{j}=\left|\hat{m}_{j}\right|$ 's are positive eigenvalues and the $\eta_{j}$ 's stand for the phases.
}

with $U_{\mathrm{TM}_{2}}^{\dagger} U_{\mathrm{TM}_{2}}=I$ and $\left|\operatorname{det} U_{\mathrm{TM}_{2}}\right|=1$. By using this mixing matrix, the Majorana neutrino mass matrix $M_{R}$ is diagonalized as

$$
\left(U_{\mathrm{TM}_{2}}^{*}\right)^{T} M_{R} U_{\mathrm{TM}_{2}}^{*}=\operatorname{diag}\left(M_{1}, M_{2}, M_{3}\right)
$$

provided the following condition holds

$\tan 2 \theta=\frac{-\sqrt{3} \epsilon}{(2 a+2 b-\epsilon) e^{i \sigma}+(2 a-2 b-\epsilon) e^{-i \sigma}}$.

By taking $\mathrm{a}, \mathrm{b}$ and $\epsilon$ complex numbers, then the reality of $\tan 2 \theta$ put a constraint on the phase $\sigma$; indeed using $a=|a| e^{i \phi_{a}}, \quad b=|b| e^{i \phi_{b}}, \quad \epsilon=|\epsilon| e^{i \phi_{\epsilon}}$ and setting $k_{a \epsilon}=$ $|a / \epsilon|, k_{b \epsilon}=|b / \epsilon|, k_{a b}=|a / b|$ as well as $\phi_{i j}=\phi_{i}-\phi_{j}$, we end up with a somehow cumbersome relation that can be simplified a little bit by taking $\phi_{b}=\phi_{\epsilon} \neq \phi_{a}$. In this case, we have

$$
m_{1}=\frac{Y_{0}^{2} v_{u}^{2}}{M_{1}}, \quad m_{2}=\frac{Y_{0}^{2} v_{u}^{2}}{M_{2}}, \quad m_{3}=\frac{Y_{0}^{2} v_{u}^{2}}{M_{3}}
$$

$\tilde{U}_{\mathrm{TM}_{2}}$ is the new neutrino mixing matrix which differs from $U_{\mathrm{TM}_{2}}$ by the interchange of the second and the third row [84]

$\tilde{U}_{\mathrm{TM}_{2}}=\left(\begin{array}{ccc}\sqrt{\frac{2}{3}} \cos \theta & \frac{1}{\sqrt{3}} & \sqrt{\frac{2}{3}} \sin \theta e^{-i \sigma} \\ -\frac{\cos \theta}{\sqrt{6}}+\frac{\sin \theta}{\sqrt{2}} e^{i \sigma} & \frac{1}{\sqrt{3}} & -\frac{\cos \theta}{\sqrt{2}}-\frac{\sin \theta}{\sqrt{6}} e^{-i \sigma} \\ -\frac{\cos \theta}{\sqrt{6}}-\frac{\sin \theta}{\sqrt{2}} e^{i \sigma} & \frac{1}{\sqrt{3}} & \frac{\cos \theta}{\sqrt{2}}-\frac{\sin \theta}{\sqrt{6}} e^{-i \sigma}\end{array}\right)$

where in the PDG parametrization, we have to add the Majorana phase matrix $\mathcal{U}_{m}=\operatorname{diag}\left(1, e^{\frac{i}{2} \alpha_{21}}, e^{\frac{i}{2} \alpha_{31}}\right)$ and where the $\alpha$ 's are related to the $\eta$ 's of footnote 4 like $\alpha_{21}=\eta_{1}-\eta_{2}$ and $\alpha_{31}=\eta_{1}-\eta_{3}$. Therefore, using this matrix $\tilde{U}_{\mathrm{TM}_{2}}$ and the definitions

$\sin \theta_{13}=\left|\tilde{U}_{e 3}\right|, \quad \sin ^{2} \theta_{23}=\frac{\left|\tilde{U}_{\mu 3}\right|^{2}}{1-\left|\tilde{U}_{e 3}\right|^{2}}$,

$\sin ^{2} \theta_{12}=\frac{\left|\tilde{U}_{e 2}\right|^{2}}{1-\left|\tilde{U}_{e 3}\right|^{2}}$

we obtain the following expressions of the three $\theta_{13}, \theta_{23}$, and $\theta_{12}$ mixing angles 


$$
\begin{aligned}
& \sin ^{2} \theta_{12}=\frac{1}{3\left(1-\sin ^{2} \theta_{13}\right)}, \quad \sin ^{2} \theta_{13}=\frac{2}{3} \sin ^{2} \theta \\
& \sin ^{2} \theta_{23}=\frac{1}{2} \pm \frac{\sin \theta_{13}\left(1-\frac{3}{2} \sin ^{2} \theta_{13}\right)^{1 / 2}}{\sqrt{2}\left(1-\sin ^{2} \theta_{13}\right)} \cos \sigma .
\end{aligned}
$$

Recall that the phase $\sigma$ is related to the Dirac phase as $\sin \sigma=-\sin 2 \theta_{23} \sin \delta_{C P}$ with [85]

$$
\cos \delta_{C P}=\frac{\cos 2 \theta_{13} \cos 2 \theta_{23}}{\sin 2 \theta_{23} \sin \theta_{13}\left(2-3 \sin ^{2} \theta_{13}\right)^{1 / 2}} .
$$

We can see from these equations that the atmospheric angle $\theta_{23}$ is related to the reactor angle $\theta_{13}$, thus, it is clear that for non zero $\theta_{13}$, the atmospheric neutrino mixing angle $\theta_{23}$ oscillates around its maximal value $-\theta_{23}=\pi / 4$ — which is in agreement with the recent result from the NOvA experiment [81]. By setting $m_{0}=Y_{0}^{2} v_{u}^{2} /|b|$, The light neutrino masses in Eq. (3.19) can be rewritten as follows

$$
\begin{aligned}
& m_{1}=m_{0} \frac{k_{b \varepsilon}}{k_{b \varepsilon}+k_{a \varepsilon}-\frac{1}{2}}, \quad m_{2}=m_{0} \frac{k_{b \varepsilon}}{k_{a \varepsilon}+1}, \\
& m_{3}=m_{0} \frac{k_{b \varepsilon}}{k_{b \varepsilon}-k_{a \varepsilon}+\frac{1}{2}}
\end{aligned}
$$

The parameters $m_{0}, k_{a \varepsilon}$ and $k_{b \varepsilon}$ are defined as three different ratios of the scalar field VEVs. A discussion concerning the ranges of these parameters will be performed in the next subsection. By using these masses, we calculate the solar $\Delta m_{21}^{2}$ and atmospheric $\Delta m_{31}^{2}$ masssquared differences, we obtain

$$
\begin{gathered}
\Delta m_{21}^{2}=m_{0}^{2} k_{b \varepsilon}^{2}\left[\frac{1}{\left(k_{a \varepsilon}+1\right)^{2}}-\frac{1}{\left(k_{b \varepsilon}+k_{a \varepsilon}-\frac{1}{2}\right)^{2}}\right] \\
\left|\Delta m_{31}^{2}\right|=m_{0}^{2} k_{b \varepsilon}^{2}\left|\frac{1}{\left(k_{b \varepsilon}-k_{a \varepsilon}+\frac{1}{2}\right)^{2}}-\frac{1}{\left(k_{b \varepsilon}+k_{a \varepsilon}-\frac{1}{2}\right)^{2}}\right|
\end{gathered}
$$

and where the two types of neutrino spectrum $\mathrm{NH}$ and $\mathrm{IH}$ are associated directly to the sign of $\Delta m_{31}^{2}$ which remains unknown. Moreover, in order to reduce the intervals of $k_{a \epsilon}=|a / \epsilon|$ and $\theta$, we plot in Fig. $1 \sin ^{2} \theta_{13}$ as a function of the deviation parameter $\theta$ and $1 / k_{a \epsilon}$ induced by the VEV of the singlet $S$. The allowed regions of $\theta$ and $1 / k_{a e}$ are constrained by the $3 \sigma$ experimental range of $\sin ^{2} \theta_{13}$, see Table IV. Thus, the restricted regions of the parameters $\theta$ and $1 / k_{a \epsilon}$ for $\mathrm{NH}(\mathrm{IH})$ are as follows

$$
\begin{aligned}
0.1726(0.1738) & \lesssim \theta \lesssim 0.1912(0.1923) \\
0.344(0.346) & \lesssim 1 / k_{a \epsilon} \lesssim 0.377(0.379)
\end{aligned}
$$

\section{B. Numerical analysis}

The neutrino oscillation experiments are known to be sensitive to the three mixing angles $\theta_{12}, \theta_{23}, \theta_{13}$ and to the neutrino mass-squared differences $\Delta m_{31}^{2}$ for atmospheric

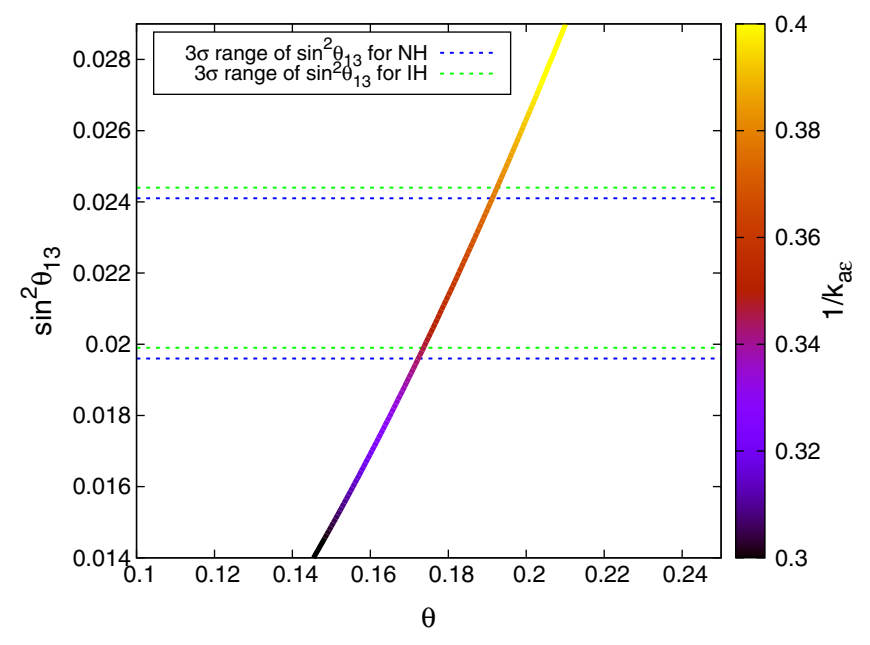

FIG. 1. The variation of $\sin ^{2} \theta_{13}$ as a function of the deviation parameter $\theta$ with the parameter $k_{a \epsilon}$ shown in the palette.

neutrinos and $\Delta m_{21}^{2}$ for solar neutrinos. The measurements of these angles as well as the mass-squared differences were reported by several global fits of neutrino data [25-27]; see Table IV. In Fig. 2, we show the correlation between the atmospheric neutrino angle $\sin ^{2} \theta_{23}$ and the solar neutrino angle $\sin ^{2} \theta_{12}$ for the normal (left) and inverted (right) mass hierarchies where the green points present all the possible model values while the blue points are the allowed model values restricted by the $3 \sigma$ ranges of the mixing angles given in Table IV. The red line in both panels corresponds to the values $\delta_{C P}=\frac{\pi}{2}, \frac{3 \pi}{2}$ which is consistent with the maximal value of the atmospheric angle. We observe that the allowed intervals for the mixing angles $\theta_{12}$ and $\theta_{23}$ are

$$
0.34 \lesssim \sin ^{2} \theta_{12} \lesssim 0.3415, \quad 0.445 \lesssim \sin ^{2} \theta_{23} \lesssim 0.598
$$

for $\mathrm{NH}$; and for $\mathrm{IH}$

TABLE IV. The best-fit values and the $3 \sigma$ ranges of neutrino oscillation parameters as reported by ref. [27] for both hierarchies.

\begin{tabular}{lccc}
\hline \hline Oscillation parameters & Ordering & Best fit & $3 \sigma$ \\
\hline $\sin ^{2} \theta_{12} / 10^{-1}$ & $\mathrm{NH} \& \mathrm{IH}$ & 3.20 & $2.73-3.79$ \\
$\sin ^{2} \theta_{23} / 10^{-1}$ & $\mathrm{NH}$ & 5.47 & $4.45-5.99$ \\
& $\mathrm{IH}$ & 5.51 & $4.53-5.98$ \\
$\sin ^{2} \theta_{13} / 10^{-2}$ & $\mathrm{NH}$ & 2.16 & $1.96-2.41$ \\
& $\mathrm{IH}$ & 2.22 & $1.99-2.44$ \\
$\Delta m_{21}^{2}\left[10^{-5} \mathrm{eV}^{2}\right]$ & $\mathrm{NH} \& \mathrm{IH}$ & 7.55 & $7.05-8.14$ \\
$\left|\Delta m_{31}^{2}\right|\left[10^{-3} \mathrm{eV}^{2}\right]$ & $\mathrm{NH}$ & 2.50 & $2.41-2.60$ \\
& $\mathrm{IH}$ & 2.42 & $2.31-2.51$ \\
$\delta^{\circ} / \pi$ & $\mathrm{NH}$ & 1.21 & $0.87-1.94$ \\
& $\mathrm{IH}$ & 1.56 & $1.12-1.94$ \\
\hline \hline
\end{tabular}



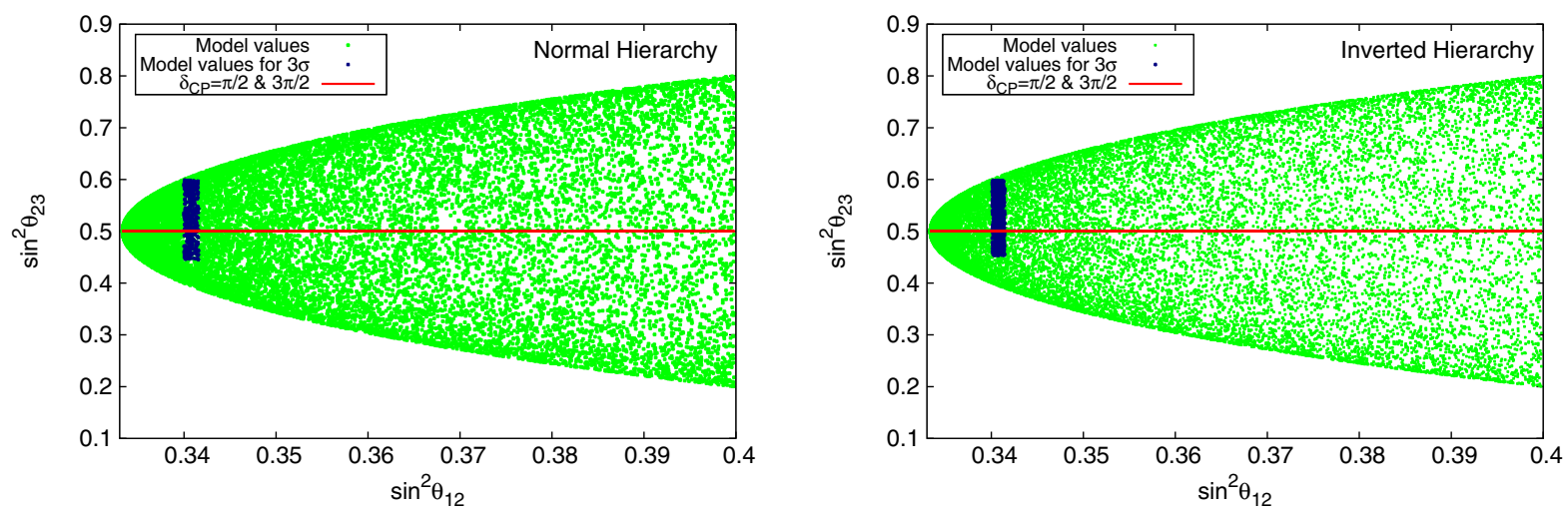

FIG. 2. Correlation plots between $\sin ^{2} \theta_{23}$ and $\sin ^{2} \theta_{12}$ for NH (left panel) and IH (right panel). The green region stands for all model values, while the blue region concerns the allowed model values for $3 \sigma$ range taken from Table IV.

$0.34 \lesssim \sin ^{2} \theta_{12} \lesssim 0.3415, \quad 0.453 \lesssim \sin ^{2} \theta_{23} \lesssim 0.597 . \quad(3.28)$

We see that the interval of $\sin ^{2} \theta_{12}$ gets more restrained compared to its $3 \sigma$ region while the intervals of $\sin ^{2} \theta_{23}$ are slightly restricted compared to their $3 \sigma$ region reported in Table IV. The same discussion applies to the correlation between the atmospheric angle $\sin ^{2} \theta_{23}$ and the reactor

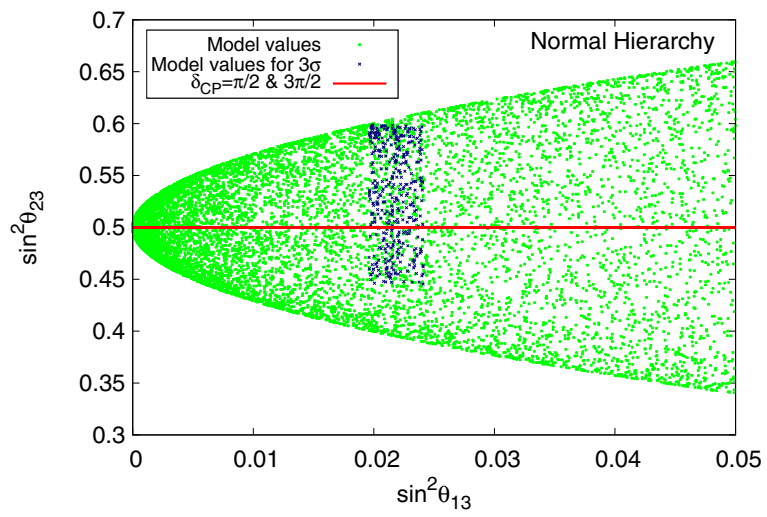

angle $\sin ^{2} \theta_{13}$ shown in Fig. 3. In this case, the obtained intervals for $\theta_{23}$ are the same as in Eq. (3.27), while the angle $\theta_{13}$ coincides with its $3 \sigma$ allowed range for $\mathrm{NH}$ and gets more restricted in the case of $\mathrm{IH}$; the range for the later case is $0.0199 \lesssim \sin ^{2} \theta_{13} \lesssim 0.0237$.

In Fig. 4, we show for both mass hierarchies the correlation among the parameters $m_{0}, k_{a b}$ and $1 / k_{b e}$ where

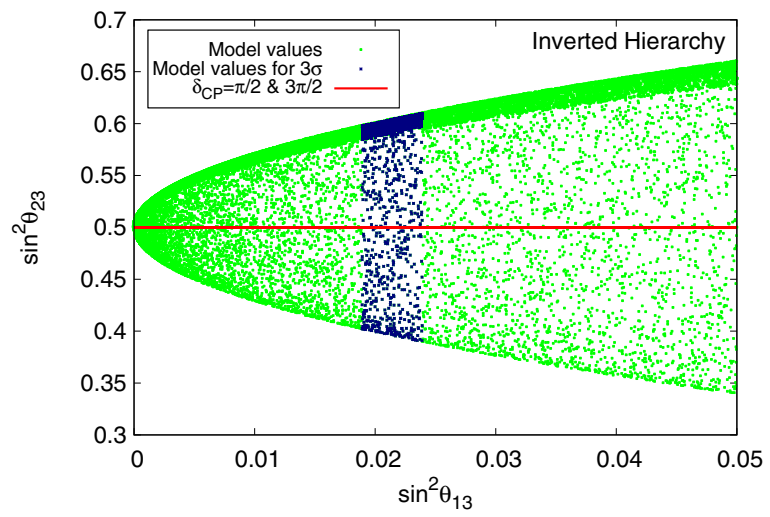

FIG. 3. Same as in Fig. 2 but for $\sin ^{2} \theta_{13}$ instead of $\sin ^{2} \theta_{12}$.
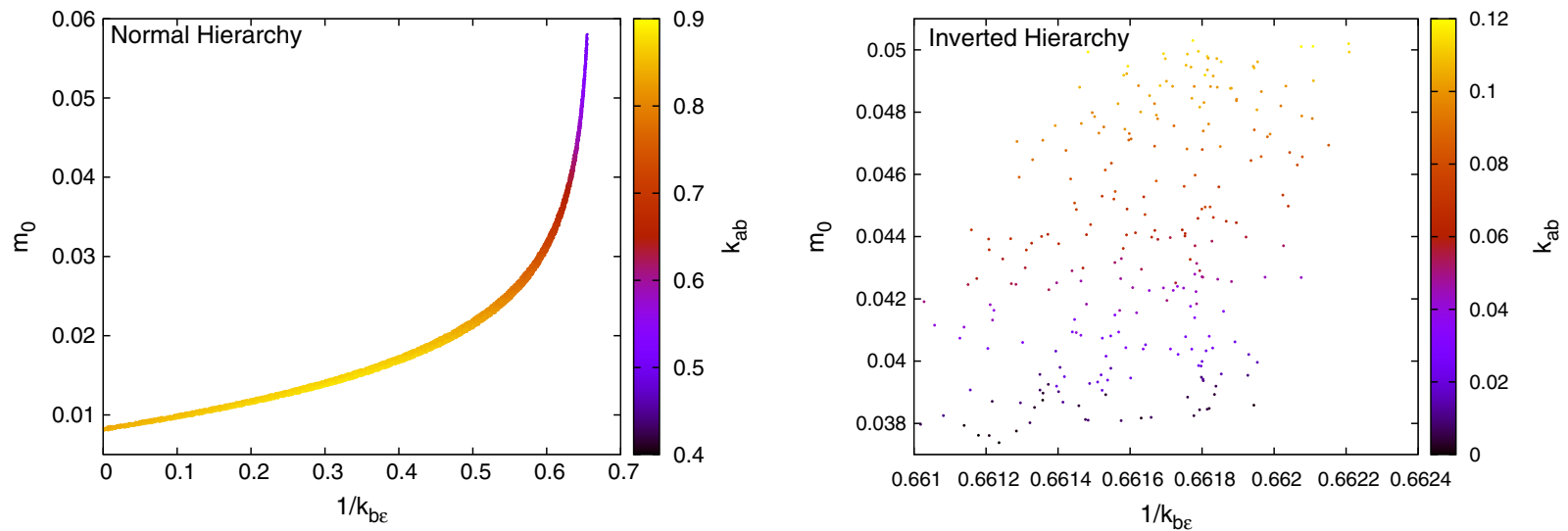

FIG. 4. Scatter plot showing the allowed regions of the $m_{0}-d-c$ parameters for the normal (left panel) and inverted (right panel) mass hierarchy. 
TABLE V. Allowed ranges of our model parameters as well as their best fit values respecting the current $3 \sigma$ ranges of the mass squared differences.

\begin{tabular}{lcc}
\hline \hline Model parameters & $\mathrm{NH}$ & $\mathrm{IH}$ \\
\hline$m_{0}[\mathrm{eV}]$ & {$\left[0.8 \times 10^{-2}, 0.058\right]$} & {$[0.037,0.05]$} \\
Best fit of $m_{0}[\mathrm{eV}]$ & $\simeq 0.0259$ & $\simeq 0.044$ \\
$k_{a b}$ & {$[0.49,0.88]$} & {$\left[0.37 \times 10^{-4}, 0.12\right]$} \\
Best fit of $k_{a b}$ & $\simeq 0.788$ & $\simeq 0.0617$ \\
$1 / k_{b e}$ & {$\left[0.16 \times 10^{-3}, 0.65\right]$} & {$[0.66,0.6622]$} \\
Best fit of $1 / k_{b e}$ & $\simeq 0.558$ & $\simeq 0.6617$ \\
\hline \hline
\end{tabular}

we used as input parameters the current $3 \sigma$ allowed range of the mass squared differences. As a result, we find that the obtained ranges are more restricted in the IH case than the $\mathrm{NH}$ case. In order to get estimations on these parameters, we take the best fit value of $\Delta m_{31}^{2}$ and $\Delta m_{21}^{2}$ and extract the corresponding values for $m_{0}, k_{a b}$ and $1 / k_{b \epsilon}$. Their intervals as well as their best fit values are as summarized in Table V. In what follows, we use these intervals as input parameters to plot the correlations between masses.

\section{PHENOMENOLOGICAL IMPLICATIONS}

In this section, we first focus on the physical observables related to experiments other than those of the neutrino oscillation experiments. These are the Majorana neutrino mass $m_{e e}$ measured in neutrinoless double $\beta$-decay experiments and the neutrino electron mass $m_{\nu_{e}}$ measured in tritium beta decay experiments. Then, we provide predictions concerning the Dirac $C P V$ phase $\delta_{C P}$ and its correlation with the Jarlskog parameter $J_{C P}$ and the atmospheric angle $\theta_{23}$.

\section{A. Neutrino mass spectrum}

We begin by investigating through scatter plots the neutrino mass spectrum where we use the recent upper bound on the sum of neutrino masses from the Planck collaboration; $\sum m_{i} \leq 0.17 \mathrm{eV}$ [62], and the $3 \sigma$ allowed ranges of the mass squared differences $\Delta m_{21}^{2}$ and $\Delta m_{31}^{2}$ given in Table IV. Moreover, in order to fix all three neutrino masses, we express the masses $m_{2}$ and $m_{3}$ in terms of the lightest neutrino mass $m_{1}$ as $\sqrt{m_{1}^{2}+\Delta m_{21}^{2}}$ and $\sqrt{m_{1}^{2}+\Delta m_{31}^{2}}$ respectively in the NH case, while in the IH case we express $m_{1}$ and $m_{2}$ in terms of the lightest neutrino mass $m_{3}$ as $\sqrt{m_{3}^{2}-\Delta m_{31}^{2}}$ and $\sqrt{m_{3}^{2}+\Delta m_{21}^{2}-\Delta m_{31}^{2}}$ respectively. Hence, we plot in Fig. 5 the exact neutrino masses $m_{1}, m_{2}$ and $m_{3}$ (denoted by the green, red and orange dots respectively) given in Eq. (3.24) and their sum $\sum m_{i}$ (denoted by the blue dots) as a function of the lightest neutrino mass ( $m_{1}$ for $\mathrm{NH}$ and $m_{3}$ for $\left.\mathrm{IH}\right)$. The neutrino oscillation parameters are taken within their currently allowed $3 \sigma$ ranges. For $\mathrm{NH}$ (left panel), the allowed range for the lightest neutrino mass is given by $m_{1} \approx 0.0043-0.043 \mathrm{eV}$, while for $\mathrm{IH}$ (right panel) the lightest neutrino mass lies in the range $m_{3} \approx$ $0.0282-0.041 \mathrm{eV}$. The allowed ranges of the remaining masses for both mass hierarchies are as summarized in Table VI. As for the sum of neutrino masses, in the normal hierarchy case-blue dots in the left panel of Fig. 5-we have $0.063 \mathrm{eV} \lesssim \sum m_{i} \lesssim 0.153 \mathrm{eV}$, while in the inverted hierarchy case-blue dots in the right panel of Fig. 5-we have $0.14 \mathrm{eV} \lesssim \sum m_{i} \lesssim 0.17 \mathrm{eV}$. From these ranges, one can suggest easily the possibility to obtain more restricted regions of the inverted hierarchy, or even exclude it if the sum of the light neutrino masses is less than $0.14 \mathrm{eV}$ in the future experiments. Indeed, strong cosmological studies ${ }^{4}$ on $\sum m_{i}$ provided the bound $\sum m_{i}<0.12 \mathrm{eV}$ at $95 \%$ C.L. [87-90] (A more recent paper provided the bound $\sum m_{i}=$ $0.11 \pm 0.03 \mathrm{eV}$ [91]). Furthermore, to determine the best estimates on the neutrino masses and their sum, we use the best fit values of the neutrino oscillation parameters given in Table IV as well as the best fit values of the parameters $m_{0}, c$, and $d$ given in Table $\mathrm{V}$. We find for $\mathrm{NH}$

$m_{1} \simeq 0.0055 \mathrm{eV}, \quad m_{2} \simeq 0.0102 \mathrm{eV}, \quad m_{3} \simeq 0.0503 \mathrm{eV}$

while for IH we find

$m_{1} \simeq 0.0606 \mathrm{eV}, \quad m_{2} \simeq 0.0612 \mathrm{eV}, \quad m_{3} \simeq 0.035 \mathrm{eV}$.

Consequently, we remark that the masses in the normal hierarchy case are lighter compared to the inverted hierarchy case, and thus our FNMSSM tends to favor the normal mass hierarchy over the inverted one. This can be seen also by calculating the best fit of $\sum m_{i}$ where we find $\sum m_{i}=0.06614 \mathrm{eV}$ for $\mathrm{NH}$ and $\sum m_{i}=0.1573 \mathrm{eV}$ for $\mathrm{IH}$. Thus, the later case is excluded if we consider the aforementioned bounds from cosmological studies.

\section{B. Absolute mass scale and the nature of neutrinos}

The neutrino oscillation experiments are insensitive to the absolute mass of neutrinos which is still unknown to this day. However, there are several nonoscillation parameters that are sensitive to the absolute neutrino mass scale, in particular, the effective neutrino electron mass $m_{\nu_{e}}$ that can be measured in tritium beta decay experiments, and the effective Majorana mass $m_{e e}$ measured in neutrinoless double beta decay experiments which will provide information on the nature of neutrinos as well.

(i) Tritium beta decay: The study of electron spectrum near its endpoint in tritium beta decay is one of the best

\footnotetext{
${ }^{4}$ For more studies on constraining the sum of the light neutrino masses in cosmological models, see Ref. [86] and the references therein.
} 

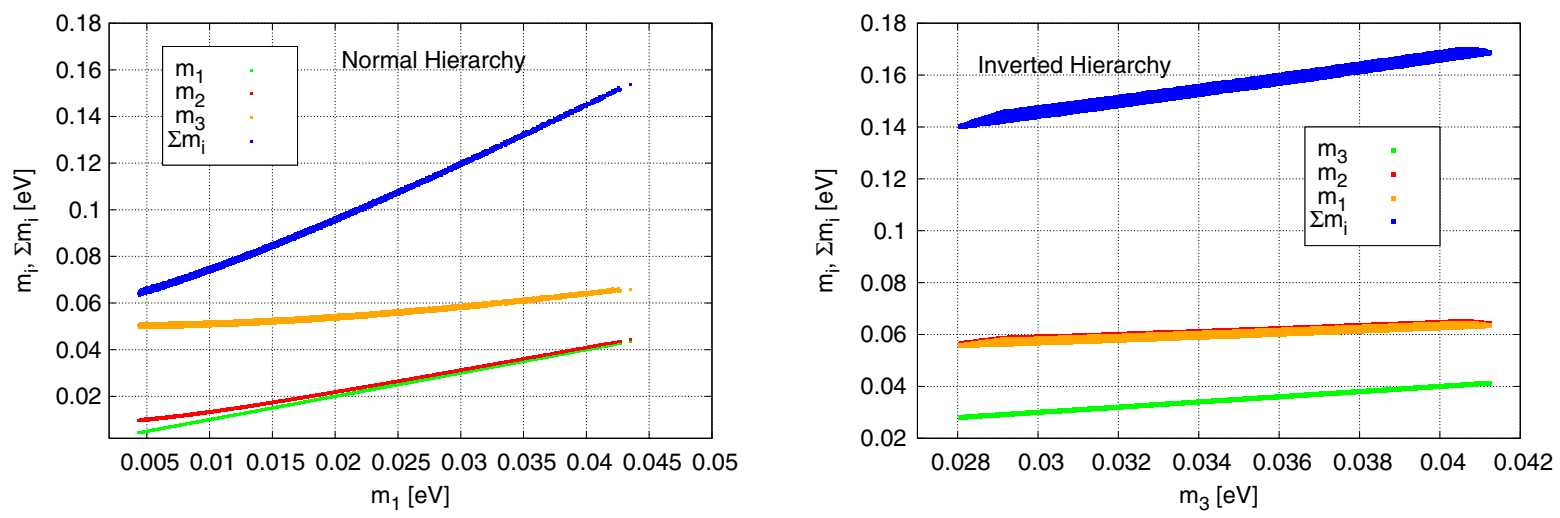

FIG. 5. Prediction for the absolute neutrino masses and their sum $\sum m_{i}$ as a function of $m_{1}\left(m_{3}\right)$ for NH (IH) in the left (right) panels. In both plots, $m_{1}, m_{2}, m_{3}$, and $\sum m_{i}$ described by green, red, orange and blue respectively.

methods to probe the neutrino mass scale. The KATRIN experiment is the current generation of direct neutrino mass measurement which is designed to measure the effective electron neutrino mass with a sensitivity of $m_{\nu_{e}}<0.2 \mathrm{eV}$ (at $90 \%$ C.L.) [92]. The effective electron neutrino mass is defined as

$$
m_{\nu_{e}}^{2}=\sum_{i}\left|U_{e i}\right|^{2} m_{i}^{2}
$$

where $m_{i}$ are the three neutrino masses and $U_{e i}$ are the elements of the first row of the PMNS matrix [93]. In our FNMSSM prototype where the mixing matrix is given by Eq. (3.20), $m_{\nu_{e}}^{2}$ is given by

$$
m_{\nu_{e}}^{2}=\frac{1}{3}\left(2 m_{1}^{2} \cos ^{2} \theta+m_{2}^{2}+2 m_{3}^{2} \sin ^{2} \theta\right)
$$

By using this equation and the mass relations for both hierarchies given after Table V, we plot in Fig. 6 the parameter $m_{\nu_{e}}$ as a function of the lightest neutrino mass. The orange region (green region) is obtained by varying all the input parameters in their $3 \sigma$ ranges for $\mathrm{NH}(\mathrm{IH})$ while our model values are presented by the blue points (the red points). As a result, we find that the effective electron neutrino mass lie in the ranges

$$
\begin{aligned}
\mathrm{NH}: & m_{\nu_{e}}(\mathrm{eV}) \in[0.00959-0.0439] ; \\
\mathrm{IH}: m_{\nu_{e}}(\mathrm{eV}) & \in[0.0554-0.0638] .
\end{aligned}
$$

TABLE VI. Our model predictions for the three light neutrino masses.

\begin{tabular}{lcc}
\hline \hline Neutrino masses & $\mathrm{NH}$ & $\mathrm{IH}$ \\
\hline$m_{1}$ & {$[0.0043-0.043]$} & {$[0.0556-0.064]$} \\
$m_{2}$ & {$[0.0094-0.044]$} & {$[0.0562-0.065]$} \\
$m_{3}$ & {$[0.0493-0.066]$} & {$[0.0282-0.041]$} \\
\hline \hline
\end{tabular}

Although the obtained intervals of $m_{\nu_{e}}$ are compatible with current data, the anticipated future sensitivity from Project 8 experiment is as low as $0.04 \mathrm{eV}$ [94], which means that if no signal is observed around this value in the future there is a good chance to exclude the inverted mass hierarchy (the smallest value of $m_{\nu_{e}}$ in our model is $0.0554 \mathrm{eV}$ ).

(ii) Neutrinoless double beta decay: The most sensitive probe of the Majorana nature of neutrinos is provided by the neutrinoless double beta decay experiments $(0 \nu \beta \beta)$ which would also provide a measurement of the absolute neutrino mass scale if it were observed. Furthermore, since the lepton number $L$ is violated in $0 \nu \beta \beta$ processes, its discovery would provide a theoretical evidence of physics beyond the SM-which conserves $L$ - and would also provide a sign of the seesaw mechanisms which imply the existence of a Majorana neutrino mass term that violates $L$. The decay rate of $0 \nu \beta \beta$ is proportional to the square of the effective Majorana mass $\left|m_{e e}\right|$ defined as

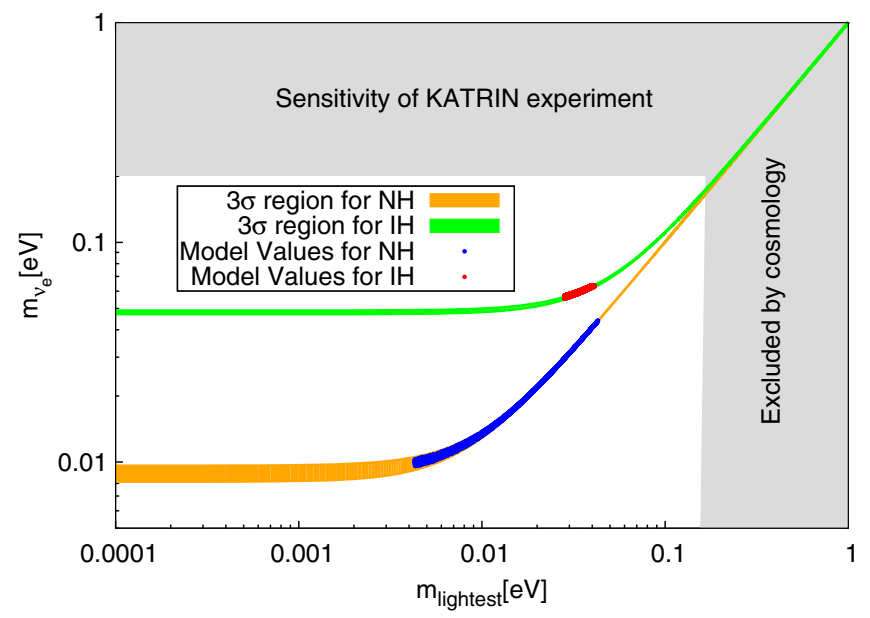

FIG. 6. The effective neutrino mass $m_{\nu_{e}}$ as function of the lightest neutrino mass $m_{i}$. The $3 \sigma$ allowed regions for $m_{\nu_{e}}$ are represented by the orange (green) colors for NH (IH). The blue and red points refer to the predicted model values for $\mathrm{NH}$ and $\mathrm{IH}$, respectively. 
$\left|m_{e e}\right|=\left|\sum_{i} U_{e i}^{2} m_{i}\right|$ where $m_{i}$ and $U_{e i}$ are as defined in the case of the effective electron mass $m_{\nu_{e}}$. In our FNMSSM, we substitute $U_{e i}^{2}$ in $\left|m_{e e}\right|$ by their expressions in the mixing matrix $\tilde{U}_{\mathrm{TM}_{2}} \times \operatorname{diag}\left(1, e^{\frac{i}{2} \alpha_{21}}, e^{\frac{i}{2} \alpha_{31}}\right)(3.20)$ where $\alpha_{21}$ and $\alpha_{31}$ are the Majorana $C P$ violating phases, we find

$$
\left|m_{e e}\right|=\frac{1}{3}\left|2 m_{1} \cos ^{2} \theta+m_{2} e^{i \alpha_{21}}+2 m_{3} \sin ^{2} \theta e^{i\left(\alpha_{31}-2 \sigma\right)}\right|
$$

Several experiments such as CUORE [95], EXO [96], NEXT [97], SuperNEMO [98], GERDA [99], and KamLAND-ZEN [100] are searching for signatures of $0 \nu \beta \beta$. The most recent bounds of $\left|m_{e e}\right|$ come from the KamLAND-Zen and GERDA experiments which are given respectively by

$$
\left|m_{e e}\right|<0.061-0.165 \mathrm{eV}, \quad\left|m_{e e}\right|<0.15-0.33 \mathrm{eV}
$$

Similar to the above discussion on $m_{\nu_{e}}$, we plot in Fig. 7 $\left|m_{e e}\right|$ as a function of the lightest neutrino mass for both mass hierarchies and we allow the Majorana phases $\alpha_{21}$ and $\alpha_{31}$ to vary in the range $[0-2 \pi]$. The cyan regions are obtained by varying all the input parameters in their $3 \sigma$ ranges, the green regions correspond to the best fit values while our model values are presented by the red points. Consequently, we find in the normal (inverted) hierarchy case that the effective Majorana mass lie in the ranges

$$
\begin{aligned}
\mathrm{NH}:\left|m_{e e}(\mathrm{eV})\right| & \in[0.007271-0.04202] ; \\
\mathrm{IH}:\left|m_{e e}(\mathrm{eV})\right| & \in[0.05519-0.0641]
\end{aligned}
$$

We see that in the normal hierarchy case the allowed range of $\left|m_{e e}\right|$ as well as its corresponding lightest neutrino mass $m_{1}$ are smaller than the ranges in the inverted hierarchy case. Furthermore, the obtained region of the effective Majorana mass in the IH case can be reached in future

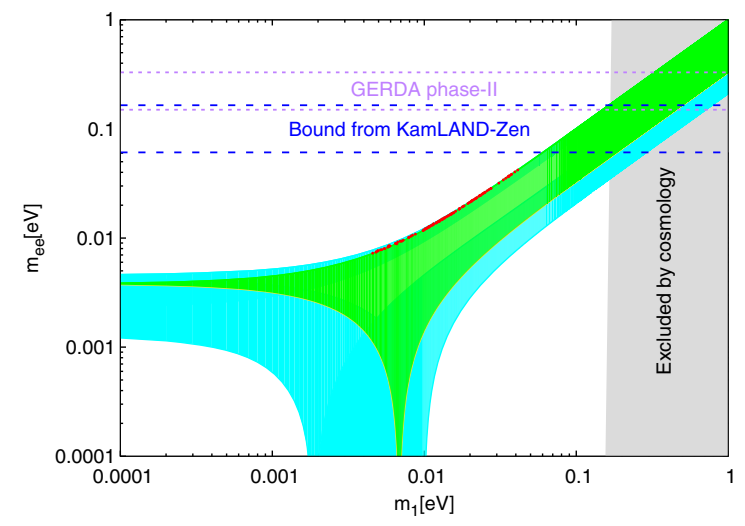

experiments like KamLAND-Zen which plans to reach a sensitivity below $0.04 \mathrm{eV}$ on $\left|m_{e e}\right|$ [101]. As for the $\mathrm{NH}$ case, the obtained lower value $(\sim 0.007 \mathrm{eV})$ is far from any current or future planed sensitivity, however, its higher values (around $0.03 \mathrm{eV}$ ) can be reached in future experiments such as EXO-200, KamLAND2-Zen and GERDA Phase II with the expected sensitivity given respectively by $(0.015-0.025) \mathrm{eV}$ [102], $0.02 \mathrm{eV}$ [103] and $(0.01-0.02) \mathrm{eV}$ [104].

\section{C. $C P V$ phase, Jarlskog invariant, and octant degeneracy}

After the discovery of the non-vanishing reactor angle, the observation of a $C P V$ in the lepton sector is now possible. One way to measure it is by means of the Jarlskog invariant parameter defined as $J_{C P}=\operatorname{Im}\left(U_{e 1} U_{\mu 1}^{*} U_{\mu 2} U_{e 2}^{*}\right)$. In the PDG standard parametrization, this parameter is expressed in terms of the three mixing angles and the Dirac $C P$ phase as follows [78]

$$
J_{C P}=\frac{1}{8} \sin 2 \theta_{12} \sin 2 \theta_{13} \sin 2 \theta_{23} \cos \theta_{13} \sin \delta_{C P}
$$

By substituting the elements of the neutrino mixing matrix by their expressions given in Eq. (3.20), the Jarlskog invariant takes a simpler form given by

$$
J_{C P}=-\frac{1}{3}\left(\sin ^{2} \theta_{23}-\frac{1}{2}\right)\left(1-\sin ^{2} \theta_{13}\right) \tan \sigma
$$

where we have expressed $\cos \theta \sin \theta$ in terms of $\sin ^{2} \theta_{13}$ and $\sin ^{2} \theta_{23}$ using Eq. (3.22). In the bottom panel of Fig. 8, we show the correlation between $\sigma$ and $\theta$ where the green (orange) points stand for $\mathrm{NH}(\mathrm{IH})$. The extracted range of $\sigma$ is given by

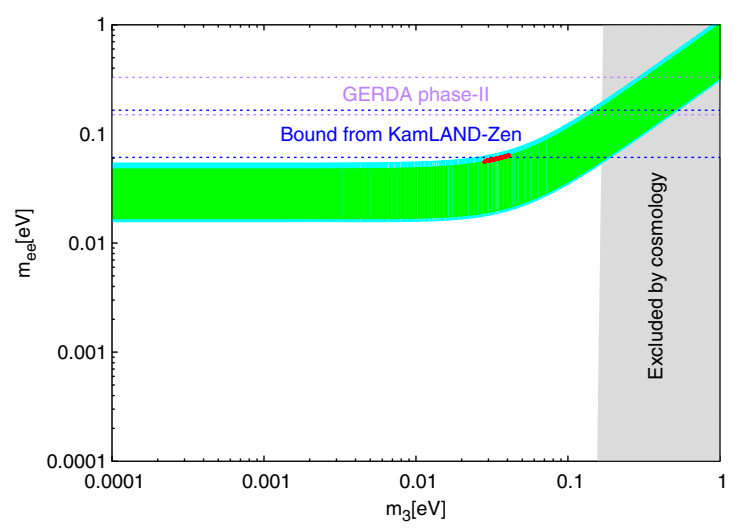

FIG. 7. $m_{e e}$ as a function the lightest neutrino mass for NH (left panel) and IH (right panel). The colored cyan regions stand for the $3 \sigma$ allowed ranges of $\left|m_{e e}\right|$ by neutrino oscillation data given in Table IV while the green colored region presents the best fit values. The horizontal dashed lines refer to the upper bound on $\left|m_{e e}\right|$ from GERDA [99] and KamLAND-ZEN [100], while the vertical gray band present the upper limit bound on the sum of the neutrino masses from Planck collaboration. The red points are our predicted model values. 

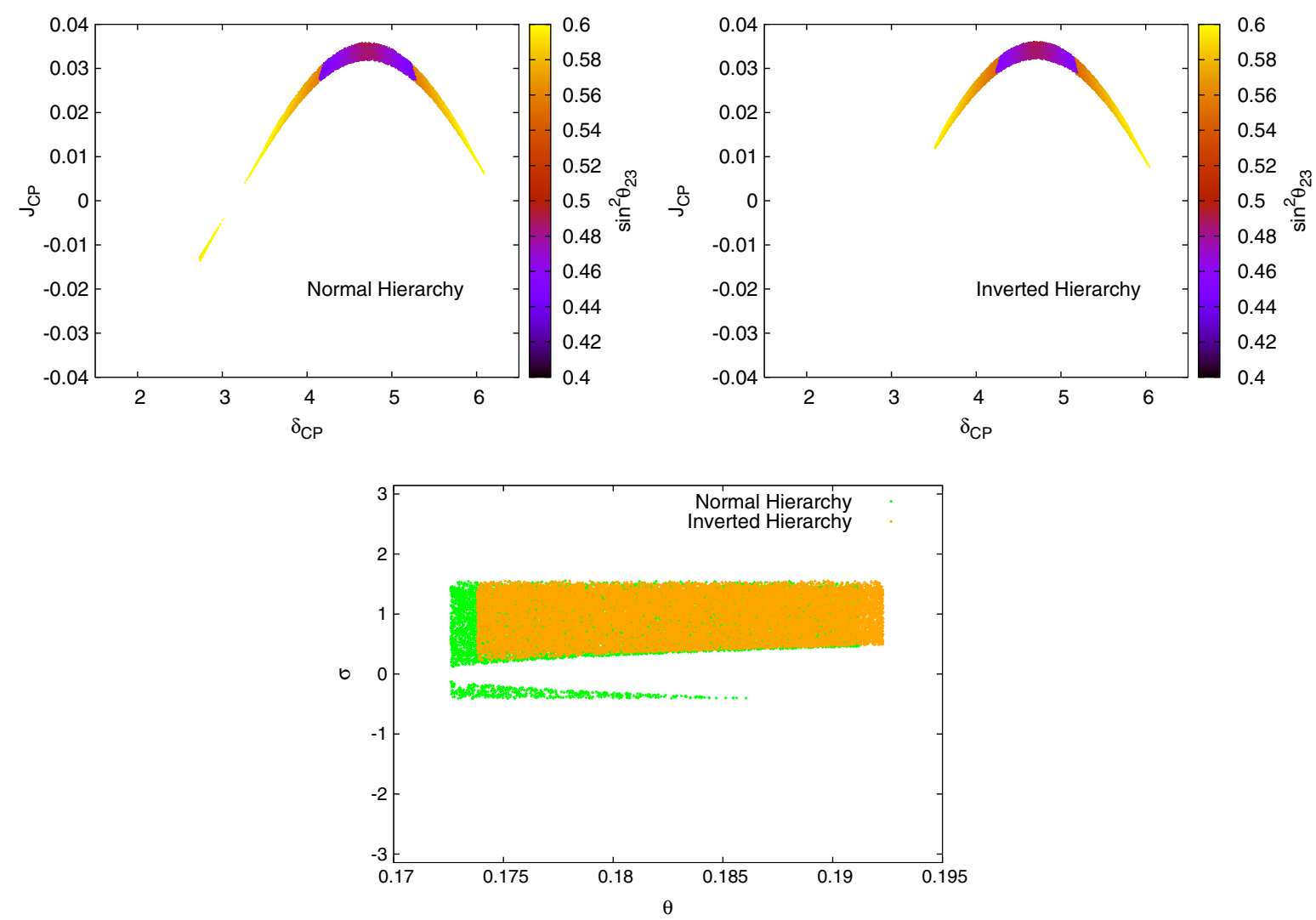

FIG. 8. This figure contains three plots; On the top, the left (right) panel represents the predicted model values for Jarlskog invariant $J_{C P}$ as a function of the $C P$-violating phase $\delta_{C P}$ with the atmospheric angle $\sin ^{2} \theta_{23}$ in the palette for NH (IH) respectively. For NH, we have both positive and negative values for $J_{C P}$; while this invariant takes only positive ones for IH. On the bottom panel, we plot the correlation between $\sigma$ and $\theta$ for $\mathrm{NH}(\mathrm{IH})$ represented by green (orange) points.

$\sigma \in\left[-0.12 \pi,-3.9 \times 10^{-2} \pi\right] \cup\left[5 \times 10^{-2} \pi, \frac{\pi}{2}[\right.$ for $\mathrm{NH}$

$\sigma \in\left[8.3 \times 10^{-2} \pi, \frac{\pi}{2}[\right.$ for $\mathrm{IH}$

It is clear from these ranges that $\sigma \neq 0 \bmod \frac{\pi}{2}$. Therefore, it is clear now from Eq. (4.10) that $J_{C P}$ cannot vanish because $\tan \sigma \neq 0$ and $\sin ^{2} \theta_{23} \neq \frac{1}{2}$, indicating that the $C P$ is always violated in our model; this result may be verified also by using the relation $\sin \sigma=-\sin 2 \theta_{23} \sin \delta_{C P}$. To illustrate this feature further, we take into account the $3 \sigma$ allowed ranges of the oscillation parameters from Table IV; and give in the left and right panels of Fig. 8 the correlations between $J_{C P}$ and $\delta_{C P}$ with $\sin ^{2} \theta_{23}$ shown in the palette for normal and inverted mass hierarchies respectively. It is clear from this figure that $J_{C P} \neq 0$ implying that $C P$ is always violated. Numerically, we find that our model predicts $\delta_{C P}$ in the ranges

$\delta_{C P} \in[0.87 \pi, 0.96 \pi] \cup[1.04 \pi, 1.5 \pi[\cup] 1.5 \pi, 1.94 \pi]$

for $\mathrm{NH}$, while it predicts the ranges

$$
\delta_{C P} \in[1.12 \pi, 1.5 \pi[\cup] 1.5 \pi, 1.93 \pi]
$$

for IH. On the other hand, the maximal value of the Jarlskog invariant is $\left|J_{C P}\right| \simeq 0.0358$ (0.036) for $\mathrm{NH}$ (IH) which is compatible with the upper bound on $\left|J_{C P}\right|_{\max } \lesssim$ 0.03599 (0.03624) obtained using the current data on the neutrino oscillation parameters [27]. It is clear from Fig. 8 that both octants of $\theta_{23}$ are permitted for both mass hierarchies; moreover, a nearly maximal atmospheric mixing $\theta_{23} \approx \frac{\pi}{4}$ favours nearly maximal $C P$ violation $\delta_{C P} \approx$ $\frac{3 \pi}{2}$ while the value of $\delta_{C P}=\pi / 2$ - strongly disfavored by current data [25] —is not permitted at all. The left panel in Fig. 8 shows that for the values around $\delta_{C P}=0.87 \pi$ and $\delta_{C P} \approx \frac{3 \pi}{2}$, the $C P$ is maximally violated when the magnitude of $J_{C P}$ is maximal $\left(J_{C P} \cong-0,0136\right.$ and $\left.J_{C P} \cong 0.0358\right)$ while in the right panel it is maximally violated $\left(J_{C P} \cong 0\right.$, 0136 and $J_{C P} \cong 0.036$ ) around the values $\delta_{C P}=1.12 \pi$ and $\delta_{C P} \approx \frac{3 \pi}{2}$.

On the other hand, the main difficulty in determining the octant sensitivity of the atmospheric angle $\theta_{23}$ arises due to the unknown value of $\delta_{C P}$. Reading from Fig. 8, the range of $\delta_{C P}$ corresponding to the $\mathrm{HO}$ for $\mathrm{NH}$ and $\mathrm{IH}$ is the same as the obtained ones in Eq. (4.12) and (4.13) respectively; while for the LO case the range of $\delta_{C P}$ corresponding to the $\mathrm{NH}(\mathrm{IH})$ is $[1.31 \pi, 1.67 \pi]([1.34 \pi, 1.65 \pi])$. 


\section{BYPASSING DOMAIN WALLS}

In this section, we study the problem of domain walls (DWs) induced by the spontaneous breaking of the discrete flavor symmetry $G_{\mathrm{f}}=\mathbb{A}_{4} \times \boldsymbol{Z}_{3}$ in the flavored NMSSM with building blocks as in Tables I-III. Then, we propose a way to bypass these domains in terms of a perturbation by higher dimensional operators suppressed by powers of the Planck scale $M_{\mathrm{Pl}}$, induced by supergravitational effect, and by using effective field action $\Gamma_{\text {eff }}$ approach.

More precisely, we first study the spontaneous breaking of the non-Abelian discrete $\mathbb{A}_{4}$ group factor of $G_{\mathrm{f}}$ down to its particular subgroups $\mathbb{Z}_{3}$ and $\mathbb{Z}_{2}$ in (2.3) since these breaking patterns are key points in the study of the DWs in the FNMSSM. Actually, the breaking $\mathbb{A}_{4} \rightarrow \mathbb{Z}_{3}$ analysis concerns the charged lepton sector and completes Eq. (2.10); while the breaking $\mathbb{A}_{4} \rightarrow \mathbb{Z}_{2}$ deals with the neutrino sector and deepens the analysis of Eqs. (3.6) and (3.7). Then, we discuss the induced domain walls in both charged and chargeless lepton sectors. After that, we provide a solution to this DW issue through the explicit breaking of the full discrete flavor group by higher dimensional operators contributing to $\Gamma_{\text {eff }}$.

\section{A. Domain walls setup}

The problem of domain walls in the framework of models based on the $\mathbb{A}_{4}$ discrete symmetry has been discussed in [105,106] and recently in [107-109]. Here we consider our FNMSSM prototype with chiral superfield content as given in Tables I-III and describe some basic ingredients to approach domain wall problem in lepton sector by using discrete symmetry group of the flavor vacua. In Secs. II and III, we showed that the generation of the lepton masses and their mixing angles arises from the spontaneous breaking of two kinds of symmetries: (i) the continuous $S U(2)_{L} \times U(1)_{Y}$ gauge symmetry by giving the VEV $v_{d}$ to the $H_{d}$ Higgs superfield as in Eq. (2.11); and (ii) the discrete flavor symmetry $G_{\mathrm{f}}=\mathbb{A}_{4} \times Z_{3}$ by giving non zero VEVs to some components of $\Phi_{i}, \Omega_{i}, S^{5}$ To handle these flavon superfields, we will imagine the sets $\Phi_{i}, \Omega_{i}, S$ as components of complex $4 \mathrm{~d}$ vector space expansions like

$$
\Phi \sim \sum_{i=1}^{3} \Phi_{i} X_{i}, \quad \Omega \sim \sum_{i=1}^{3} \Omega_{i} X_{i}, \quad S \sim S X_{4}
$$

with $X_{i}$ 's standing for a system of vector basis with some flavon space metric $h_{i j}=\left\langle X_{i} \mid X_{j}\right\rangle$; the first three directions $\left(X_{1}, X_{2}, X_{3}\right)$ are for the $A_{4}$ - triplets and the fourth $X_{4}$ for singlets. As these chiral superfields - collectively denoted below by $\Upsilon$-play an important role in the analysis of

\footnotetext{
${ }^{5}$ Below we will mainly focus on the properties of the nonAbelian part $\mathbb{A}_{4}$ of the flavor symmetry $G_{f}$; the Abelian factor $\boldsymbol{Z}_{3}$ can be implemented in a straightforward manner.
}

DWs, we start by giving three interesting comments that will be used to approach DWs in the "flavon" field space. The first comment concerns the above formal $4 \mathrm{~d}$ - vector expansions (5.1) which are complex valued developments while we will need real expansions when looking for representing DWs by real quivers. The two other comments concern useful features regarding the structure of the DWs viewed from space- time as well as their properties with respect to subgroups of $G_{\mathrm{f}}$.

(i) First comment: flavon scalar potential and real VEV quivers

The superfields we are using here are chiral super functions $\Upsilon=\xi_{\Upsilon}+\theta \psi_{\Upsilon}+\theta^{2} F_{\Upsilon}$ carrying in general a quantum number of $G_{\mathrm{f}}$ but no gauge charge restricting therefore the gauge invariant kinetic energy density $\sum\left|\mathcal{D}_{\mu} \xi_{\Upsilon}\right|^{2}$ just to the ordinary $\sum\left|\partial_{\mu} \xi_{\Upsilon}\right|^{2}$ with no couplings with vector gauge potentials $\mathrm{A}_{\mu}^{s u_{2} \times u_{1}}$. However, there are interactions-among themselves and with other chiral superfields of the model - governed by $G_{\mathrm{f}}$ and contributing to the full classical scalar potential $\mathcal{V}_{\text {tot }}$ of the FNMSSM by some real function of the $\xi_{\Upsilon}$ scalar fields to which we refer hereafter to as

$$
\begin{aligned}
\tilde{\mathcal{V}} & =\tilde{\mathcal{V}}\left(\xi_{\Phi}, \xi_{\Omega}, \xi_{S} ; \ldots\right) \\
& :=\tilde{\mathcal{V}}\left(\xi_{\Upsilon}, \bar{\xi}_{\Upsilon}\right)
\end{aligned}
$$

and whose supersymmetric part can be read from $\sum\left|F_{\Upsilon}\right|^{2}$. Recall that, the flavor symmetry $G_{\mathrm{f}}$ has been implemented by adding an index to the flavon superfields like $\Upsilon_{i}$; for example the $\Phi$ flavon is a triplet under $\mathbb{A}_{4} \subset G_{\mathrm{f}}$; so it involves three chiral superfields $\Phi_{1}, \Phi_{2}, \Phi_{3}$ that couple to each other and to other chiral superfield of the theory in $G_{\mathrm{f}}$-invariant manner like in (2.5). Notice also that because of supersymmetry the superfields in the expansion Eq. (5.1) are complex chiral superfields, and their scalar components; in particular the lowest $\xi_{\Upsilon} \sim \sum \xi_{\Upsilon_{i}} X_{i}$-which may have VEVs $\left\langle\xi_{\Upsilon_{i}}\right\rangle$ that can be used to break symmetries-are also complex quantities. This complex nature of field variables is somehow a disturbing thing for later use; especially when searching a real graphic representation of domain walls. To work around this difficulty, it is interesting to use $\mathbb{C} \sim \mathbb{R}^{2}$ and split the complex flavon scalars $\xi_{\Upsilon}$ and the $X_{k}$-generators like $\xi=\operatorname{Re} \xi+i \operatorname{Im} \xi$ and

$$
X_{k} \sim\left(\begin{array}{c}
U_{k} \\
0
\end{array}\right), \quad i \otimes X_{k} \sim\left(\begin{array}{c}
0 \\
V_{k}
\end{array}\right), \quad k=1,2,3,4
$$

where $U_{k}$ and $V_{k}$ are real $4 \mathrm{~d}$ vectors. With this real splitting, we can put the above complex $4 \mathrm{~d}$ expansions 
of $\mathbb{A}_{4}$ - flavonic representations with respect to $X_{i}$ 's as real 8-dimensional vectors like

$$
\xi_{\Upsilon} \sim \sum_{k}\left(\operatorname{Re} \xi_{\Upsilon_{k}}\right) U_{k}+\sum_{k}\left(\operatorname{Im} \xi_{\Upsilon_{k}}\right) V_{k} .
$$

For example, the real VEV $v_{\Phi}$ of the flavon $\Phi$ used in the derivation of Eq. (2.10) may be imagined as the modulus of a real $6 \mathrm{~d}$ vector in $\mathbb{R}^{6} \subset \mathbb{R}^{8}$ generated by (5.3). Then, geometrically speaking, the $\operatorname{VEV}\langle\Phi\rangle$ can be represented by a vector $\varphi_{1} U_{1}$ pointing in the first real $U_{1}$ direction of the $\left(U_{k}, V_{k}\right)$ vector space. The actions on $\langle\Phi\rangle=\varphi_{1} U_{1}:=\varphi$ by the two generators $\mathcal{S}$ and $\mathcal{T}$ of the flavor group $\mathbb{A}_{4}$ generate in general a polygon in the $\left(U_{k}, V_{k}\right)$ space with 12 vertices given by

$\varphi, \quad \mathcal{S} \mathcal{T} \varphi, \quad \mathcal{T} \mathcal{S} \mathcal{T} \varphi, \quad \mathcal{T}^{2} \mathcal{S} \mathcal{T} \varphi, \quad \mathcal{S} \varphi, \quad \mathcal{T} \mathcal{S} \varphi$

$\mathcal{S} \mathcal{T} S \varphi, \quad \mathcal{T}^{2} \mathcal{S} \varphi, \quad \mathcal{T} \varphi, \quad \mathcal{T}^{2} \varphi, \quad \mathcal{S T}^{2} \varphi, \quad \mathcal{T S}^{2} \varphi$

This polygonal graph may be interpreted as the DW quiver viewed from the flavon space $\mathfrak{F}$ with completely broken $\mathbb{A}_{4}$. Notice that for the flavon triplet $\Phi$, this $\mathfrak{F}$ space is parametrized by the scalar fields $\xi_{\Phi_{1}}, \xi_{\Phi_{2}}, \xi_{\Phi_{3}}$ and so is isomorphic to $\mathbb{C}^{3} \simeq \mathbb{R}^{6}$. Notice also that DW quivers we are concerned with below correspond to partial breaking of $\mathbb{A}_{4}$ down to a subgroup $H_{f}$; and then do not have 12 vertices; but a lower number given by the order of the broken part of the $\mathbb{A}_{4}$ symmetry denoted by $K_{f}$. In fact we will encounter two kinds of real quivers that are associated with two particular spontaneous breaking patterns namely: $\mathbb{A}_{4}$ down to $\mathbb{Z}_{3}$, and $\mathbb{A}_{4}$ down to $\mathbb{Z}_{2} ;$ by using $\mathbb{A}_{4} \cong \mathbb{V}_{4} \rtimes \mathbb{Z}_{3}$, the broken parts of these breakings are respectively given by $\mathbb{V}_{4}$ and $\mathbb{Z}_{2} \rtimes \mathbb{Z}_{3}$ [107]. For the first breaking we have $\mathcal{T}\langle\Phi\rangle=\langle\Phi\rangle$, then the above 12 vertices reduce to 4 ones defining the vertices of a tetrahedronal quiver in $\mathfrak{F}$ (see subsection V B); and for the second breaking, we have $\mathcal{S}\langle\Phi\rangle=\langle\Phi\rangle$ and then the 12 vertices reduce to 6 vertices defining an octahedronal quiver (see subsection $\mathrm{V} \mathrm{C}$ ).

(ii) Second comment: Quantum numbers and domain walls

The quantum numbers $n_{G_{\mathrm{f}}}$ of the flavon superfields under the non-Abelian discrete symmetry $G_{f}$ were given in Tables II-III in terms of the characters of the representation group generators $\mathcal{S}$ and $\mathcal{T}$. Recall that the two flavons $\Phi$ and $\Omega$ transform in the same $3_{(-1,0)}$ representation of $\mathbb{A}_{4}$, but they are distinguished by the extra discrete $\boldsymbol{Z}_{3}$. The $S$ is a non trivial singlet under both $\mathbb{A}_{4}$ and $\boldsymbol{Z}_{3}$, and so a linear term of this superfield in the effective superpotential $\mathcal{W}_{\text {eff }}(\Phi, \Omega, S)$ breaks completely the $G_{\mathrm{f}}$ flavor symmetry. This $S$ - property will be used later on in overcoming the domain wall problem in the neutrino sector by using effective scalar potential approach; see subsection V D for details. Moreover, under the breaking of $G_{\mathrm{f}}$ down to a given subgroup $H_{f}$; these $n_{G_{f}}$ quantum numbers get split to two subsets $n_{H_{f}}$ and $n_{K_{f}}$; the $n_{H_{f}}$ for $H_{f}$ and the $n_{K_{f}}$ for the broken subsymmetry; the second subset $n_{K_{f}}$ is important for describing the DWs; it will be used to deal with the two following things:

(a) the indexing of the split degenerate vacua $\left\langle\xi_{\Upsilon}\right\rangle_{a}$ of the potential $\tilde{\mathcal{V}}\left(\xi_{\Upsilon}, \bar{\xi}_{\Upsilon}\right)$; for convenience, we shall denote these degenerate vacua like $\left\langle\xi_{\Upsilon}\right\rangle_{a}: \equiv \varphi_{a}$ with values for the subindex $a=1, \ldots, n_{K_{f}}$. For the example of $H_{f}=\mathbb{Z}_{3}$, the index $a$ takes the values 1,2,3,4; and for $H_{f}=\mathbb{Z}_{2}$, it takes the values $1, \ldots, 6$.

(b) the study of the properties of the network of domain walls viewed from the flavon space $\mathfrak{F}$; in particular the $l_{a b}$ DWs interpolating between the various $\varphi_{a}$ - vacua whose space time description correspond to [20-23]

$$
l_{a b}=x\left(\varphi_{b}\right)-x\left(\varphi_{a}\right)
$$

This space time quantity $l_{a b}$ can be interpreted as the width ${ }^{6}$ of the DWs since it vanishes for $\varphi_{a}=\varphi_{b}$; i.e.: $l_{a a}=0$. A simple explicit expression in terms of the fields $\xi_{\Upsilon}$ and $\bar{\xi}_{\Upsilon}$ that defines the above $l_{a b}$ DWs is given by the following integral formula in the space $\mathfrak{F}$ of flavon scalars,

$l_{a b}= \pm \int_{\varphi_{a}}^{\varphi_{b}} d x, \quad d x=\frac{d \eta_{\Upsilon}}{\sqrt{\mathcal{E}+\tilde{\mathcal{V}}\left(\xi_{\Upsilon}, \bar{\xi}_{\Upsilon}\right)}}$

with $\mathcal{E}$ is a constant standing for the energy density of a static flavon configuration $\xi_{\Upsilon}$; and where $\left(d \eta_{\Upsilon}\right)^{2}=d \xi_{\Upsilon} d \bar{\xi}_{\Upsilon}$ is the metric ${ }^{7}$ in the $\mathfrak{F}$ space parameterised by $\xi_{\Upsilon}, \bar{\xi}_{\Upsilon}$; see Eq. (5.12) below. A short way to derive this $l_{a b}$ interpolating relation is to use the $4 \mathrm{~d}$ space time flavon dynamics described by the following Lagrangian density

\footnotetext{
${ }^{6}$ For a consistent interpretation of $l_{a b}$ as a space-time width, we need finite values of the fields at space infinity. This requires however introducing some input parameters in the scalar potential that controls the finitude of value of the fields at space infinity as remarkably done in; see also $[106,108]$ for other approaches. We suspect that these methods might be applied as well to our construction provided implementation of extra nonrenormalizable terms in the superpotential although technically is more laborious.

${ }^{7}$ In complex space $\mathbb{C}^{n}$ with $\mathrm{n}$ coordinates $z_{l}=x_{l}+i y_{l}$, the metric is given by $d s^{2}=\sum_{l} d z_{l} d \bar{z}_{l}$ and reads with real variables as $\sum_{l}\left(d x_{l}\right)^{2}+\left(d y_{l}\right)^{2}$.
} 


$$
\mathcal{L}_{4 d}=\partial_{\mu} \bar{\xi}_{\Upsilon} \partial^{\mu} \xi_{\Upsilon}-\tilde{\mathcal{V}}\left(\xi_{\Upsilon}, \bar{\xi}_{\Upsilon}\right)
$$

and look for a relation that links the space time differentials $d x^{\mu}$ and the flavon potential $\tilde{\mathcal{V}}\left(\xi_{\Upsilon}, \bar{\xi}_{\Upsilon}\right)$. From the above $\mathcal{L}_{4 d}$ one can derive the flavon field equations of motion that we express like

$$
\ddot{\xi}_{\Upsilon}-\vec{\nabla}^{2} \xi_{\Upsilon}+\frac{\partial \tilde{\mathcal{V}}}{\partial \bar{\xi}_{\Upsilon}}=0
$$

together with the complex conjugate. By considering time independent flavon field configurations; i.e.: $\dot{\xi}_{\Upsilon}=0$, and restricting the static $3 \mathrm{~d}$ space to one space direction-planar DWs-by setting $\xi_{\Upsilon}(t, x, y, z) \equiv \zeta_{\Upsilon}(x)$ with no $y$ nor $z$ dependence, the above $\mathcal{L}_{4 d}$ Lagrangian density reduces to $\mathcal{L}_{1 d}=-\bar{\zeta}_{\Upsilon}^{\prime} \zeta_{\Upsilon}^{\prime}-\tilde{\mathcal{V}}\left(\zeta_{\Upsilon}, \bar{\zeta}_{\Upsilon}\right)$. In this case, the $1 \mathrm{~d}$ - field equations of motion resulting from $\mathcal{L}_{1 d}$ read as follows

$\frac{d^{2} \zeta_{\Upsilon}}{d x^{2}}-\frac{\partial \tilde{\mathcal{V}}}{\partial \bar{\zeta}_{\Upsilon}}=0, \quad \frac{d^{2} \bar{\zeta}_{\Upsilon}}{d x^{2}}-\frac{\partial \tilde{\mathcal{V}}}{\partial \zeta_{\Upsilon}}=0$

By multiplying the first equation by $\frac{d \bar{\zeta}_{\Upsilon}}{d x}$ and the second one by $\frac{d \zeta_{\Upsilon}}{d x}$; then, adding the two obtained relations, we end with a conserved expression $\frac{d \mathcal{E}}{d x}=0$ with energy density like

$$
\mathcal{E}=\left(\frac{d \bar{\zeta}_{\Upsilon}}{d x}\right)\left(\frac{d \zeta_{\Upsilon}}{d x}\right)-\tilde{\mathcal{V}}
$$

This conserved expression leads in turn to a relationship between the $d x$ differential in $\mathrm{x}$ - space and the $d \eta_{\Upsilon}$ differential in the $\zeta_{\Upsilon}$ - field space; it read as $(\mathcal{E}+\tilde{\mathcal{V}}) d x^{2}=d \eta_{\Upsilon}^{2}$ with $d \eta_{\Upsilon}^{2}=$ $d \bar{\zeta}_{\Upsilon} d \zeta_{\Upsilon}$ and; by taking the square root, we then have

$$
d x= \pm \frac{d \eta_{\Upsilon}}{\sqrt{\mathcal{E}+\tilde{\mathcal{V}}}}
$$

which by integration between two given vacua $\left(\varphi_{a}, \varphi_{b}\right)$ of the scalar potential, we discover (5.7). Later on, we shall think of the simple expression $\frac{d \eta_{\Upsilon}}{\sqrt{\mathcal{E}+\tilde{\mathcal{V}}}}$ in (5.12) as a typical Hermitian 1 -form $\varpi_{1}=\frac{1}{\sqrt{\mathcal{E}+\tilde{\mathcal{V}}}} d \eta_{\Upsilon}$ in the flavon space $\mathfrak{\mho}$ that characterizes the DWs interpolating between two vacua $\varphi_{a b}=\left[\varphi_{a}, \varphi_{b}\right]$. So, we can also express (5.7) by using $\varpi_{1}$ language like

$$
l_{a b}= \pm \int_{\left[\varphi_{a}, \varphi_{b}\right]} \varpi_{1}
$$

that is an integral of the 1 -form $\varpi_{1}$ on a compact line $\varphi_{a b}$ with boundaries given by the critical points of the flavon potential. This formula is suggestive in the sense that permits to think of generic DWs extending between p- vacua in $\mathfrak{F}$ as given by multi-integrations over $\varpi_{p-1}$ forms on the flavon space $\mathfrak{F}$; for the cases of $p=3$ and $p=4$, see Eqs. (5.34), (5.36), and (5.37) given below.

(iii) Third comment: $\mathbb{V}_{4}$ and $\mathbb{Z}_{2} \rtimes \mathbb{Z}_{3}$

The order of the discrete alternating $\mathbb{A}_{4}$ is equal to 12 ; the same as the product $4 \times 3$ which is the order of semidirect product of $\mathbb{V}_{4} \rtimes \mathbb{Z}_{3}$ where $\mathbb{V}_{4}$ is the Klein group. This order four group $\mathbb{V}_{4}$ is Abelian and can be expressed like the product of two $\mathbb{Z}_{2}$ copies; that is $\mathbb{V}_{4} \sim \mathbb{Z}_{2} \times \mathbb{Z}_{2}$. To distinguish these two $\mathbb{Z}_{2}$ copies, we shall think of $\mathcal{S}$ as the generator of the first $\mathbb{Z}_{2}$ and use a different $\mathcal{S}^{\prime}$ to refer to the generator of the second copy that we denote like $\mathbb{Z}_{2}^{\prime}$. Notice that according to Eq. (2.3), there exists a third $\mathbb{Z}_{2}^{\prime \prime}$ subgroup inside the alternating $A_{4}$ whose generator will be denoted below as $\mathcal{S}^{\prime \prime}$ and is related to the generators of the two other $\mathbb{Z}_{2}$ 's like $\mathcal{S}^{\prime \prime}=\mathcal{S} \mathcal{S}^{\prime}=\mathcal{S}^{\prime} \mathcal{S}$.

In the basis where $\mathcal{T}$ is diagonal; i.e.: $\mathcal{T}=$ $\operatorname{diag}(1, \bar{\omega}, \omega)$, the matrix representations of these $\mathcal{S}, \mathcal{S}^{\prime}$, and $\mathcal{S}^{\prime \prime}$ are given by

$$
\begin{aligned}
& \mathcal{T}=\left(\begin{array}{lll}
1 & 0 & 0 \\
0 & \bar{\omega} & 0 \\
0 & 0 & \omega
\end{array}\right), \quad \mathcal{S}=\frac{1}{3}\left(\begin{array}{ccc}
-1 & 2 & 2 \\
2 & -1 & 2 \\
2 & 2 & -1
\end{array}\right) \\
& \mathcal{S}^{\prime}=\frac{1}{3}\left(\begin{array}{lll}
-1 & 2 \omega & 2 \bar{\omega} \\
2 \bar{\omega} & -1 & 2 \omega \\
2 \omega & 2 \bar{\omega} & -1
\end{array}\right), \quad \mathcal{S}^{\prime \prime}=\frac{1}{3}\left(\begin{array}{ccc}
-1 & 2 \bar{\omega} & 2 \omega \\
2 \omega & -1 & 2 \bar{\omega} \\
2 \bar{\omega} & 2 \omega & -1
\end{array}\right)
\end{aligned}
$$

satisfying the properties $\mathcal{S}^{2}=\mathcal{S}^{\prime 2}=\mathcal{S}^{\prime \prime 2}=I$ and

$$
\mathcal{S}^{\prime}=\mathcal{T} \mathcal{S} \mathcal{T}^{-1}, \quad \mathcal{S}^{\prime \prime}=\mathcal{T}^{2} \mathcal{S} \mathcal{T}^{-2} .
$$

Observe that the sum of entries of the rows of $\mathcal{S}$ and $\mathcal{S}^{\prime}$ matrices are equal; $\sum_{j} \mathcal{S}_{i j}=1$ and $\sum_{j} \mathcal{S}_{i j}^{\prime}=-1$, two properties that will be useful later on when studying the breaking of $\mathbb{A}_{4}$ in the neutrino sector. Observe also that by giving a VEV to the complex $\Phi$ - flavon like ${ }^{8}$

$$
\langle\Phi\rangle=v_{\Phi}\left(\begin{array}{l}
1 \\
0 \\
0
\end{array}\right)
$$

\footnotetext{
${ }^{8}$ In the real basis (5.3), this VEV may be imagined as $\langle\Phi\rangle^{T}=\left(v_{\Phi}, 0,0,0,0,0\right)$.
} 
the alternating $\mathbb{A}_{4}$ gets broken down to a subgroup $\mathbb{Z}_{3}$ with broken part given by the Klein group $\mathbb{V}_{4}$ that characterizes the split vacua. The breaking $\mathbb{A}_{4} \rightarrow \mathbb{Z}_{3}$ is explicitly exhibited on the matrix representation (5.14) of the generators of $\mathbb{A}_{4}$ which shows that

$$
\begin{array}{rlrl}
\mathcal{T}\langle\Phi\rangle & =\langle\Phi\rangle, & & \mathcal{T}^{3}=I_{i d} \\
\mathcal{S}\langle\Phi\rangle \neq\langle\Phi\rangle, & & \mathcal{S}^{2}=I_{i d} \\
\mathcal{S}^{\prime}\langle\Phi\rangle & \neq\langle\Phi\rangle, & & \mathcal{S}^{\prime 2}=I_{i d}
\end{array}
$$

where $\mathcal{T}$ generates $\mathbb{Z}_{3}$. With this choice of VEV, one gives masses to the charged leptons that are proportional to $\frac{v_{\Phi}}{\Lambda}$ as shown in the superpotential (2.10); but also induces DWs to be studied with details in next subsection. If instead of $\Phi$, we give a non zero VEV to the $\Omega$ - flavon by choosing the three VEVs equal like ${ }^{9}$

$$
\langle\Omega\rangle=v_{\Omega}\left(\begin{array}{l}
1 \\
1 \\
1
\end{array}\right)
$$

the discrete $\mathbb{A}_{4}$ symmetry group gets broken down to a $\mathbb{Z}_{2}$ subsymmetry with broken part behaving like $\mathbb{Z}_{3} \rtimes \mathbb{Z}_{2}$; as there is no symmetric $\mathbb{S}_{3}$ inside $\mathbb{A}_{4}$. This breaking may be directly checked from Eq. (5.14) showing that

$$
\begin{array}{rlrl}
\mathcal{S}\langle\Omega\rangle & =\langle\Omega\rangle, & & \mathcal{S}^{2}=I_{i d} \\
\mathcal{T}\langle\Omega\rangle \neq\langle\Omega\rangle, & & \mathcal{T}^{3}=I_{i d} \\
\mathcal{S}^{\prime}\langle\Omega\rangle \neq\langle\Omega\rangle, & & \mathcal{S}^{\prime 2}=I_{i d}
\end{array}
$$

with $\mathcal{S}$ generating $\mathbb{Z}_{2}$. Notice that the breaking of $\mathbb{A}_{4}$ down to a $\mathbb{Z}_{2}$ can be also realized by the nontrivial $\mathbb{A}_{4}$ - singlet $S$ whose VEV $v_{S}$ preserves $\mathbb{Z}_{2}$ since, according to Table III based on the characters of $\mathcal{S}$ and $\mathcal{T}$, we have

$$
\mathcal{S}\langle S\rangle=\langle S\rangle, \quad \mathcal{T}\langle S\rangle=e^{\frac{2 i \pi}{3}}\langle S\rangle
$$

In what follows, we study separately these two breaking patterns $\mathbb{A}_{4} \rightarrow \mathbb{Z}_{3}$ (for charged leptons) and $\mathbb{A}_{4} \rightarrow \mathbb{Z}_{2}$ (for neutrinos); they are respectively realised by the VEVs of the flavon triplets $\Phi$ and $\Omega$. As an ultimate goal of this study, we aim to describe with details the induced domain walls in the flavored NMSSM with building blocks as in Tables I-III and provide a solution for the neutrino sector where DWs are inevitable.

\footnotetext{
${ }^{9}$ In the real basis (5.3), this VEV is given by $\langle\Omega\rangle^{T}=$ $v_{\Omega}(1,0,1,0,1,0)$.
}

\section{B. Domain walls in charged lepton sector}

In this subsection we study the domain walls in the charged lepton sector in the flavored NMSSM which are induced by the breaking $\mathbb{A}_{4} \rightarrow \mathbb{Z}_{3}$. We will show that the DWs in this sector are not problematic due to our estimation of the lower bound of $v_{\Phi}$ VEV which happens to be greater than the inflationary scale which is around $10^{14} \mathrm{GeV}$. To that purpose, we first describe the properties of the building blocks in our flavored NMSSM prototype after the $\mathbb{A}_{4} \rightarrow \mathbb{Z}_{3}$ breaking. Then, we analyse the properties of the split degenerate vacua in the space $\mathfrak{F}$ of flavons as well as the structure of the induced domain walls.

\section{Breaking pattern of $\mathbb{A}_{4}$ to $\mathbb{Z}_{3}$}

We start from the list of Eq. (2.3) concerning the discrete alternating $\mathbb{A}_{4}$ group that acts on a given four- states system say $\left\{\left|X_{1}\right\rangle, \ldots,\left|X_{4}\right\rangle\right\}$. From this table, we learn that $\mathbb{A}_{4}$ may be spontaneously broken down to any one of the subgroups in (2.3) by choosing appropriate VEV directions. In the case where $\mathbb{A}_{4}$ is broken down to one of the four possible $\mathbb{Z}_{3}$ 's living inside of $\mathbb{A}_{4}$; say to the subgroup given by

$$
\mathbb{Z}_{3}=\left\{I_{i d},(123),(132)\right\}
$$

with point $\left|X_{4}\right\rangle$ fixed, only the $\mathcal{T}$ generator of the $\mathbb{Z}_{3}$ subsymmetry of $\mathbb{A}_{4}$ survives; the other $\mathcal{S}$ generator gets broken. By taking $\mathcal{T}=(132)$; then we have $(123)=$ $\mathcal{T}^{-1}=\mathcal{T}^{2}$ and so $\mathbb{Z}_{3}$ is just the set

$$
\mathbb{Z}_{3}=\left\{I, \mathcal{T}, \mathcal{T}^{2}\right\}
$$

with $\mathcal{T}^{3}=I$. In this picture, $\mathcal{T}$ can be represented by a diagonal $3 \times 3$ matrix with eigenvalues $(1, \bar{\omega}, \omega)$ and its typical eigenvectors $X_{q}^{\prime}$ are respectively given by

$$
\begin{aligned}
& X_{1}^{\prime}=X_{1}+X_{2}+X_{3} \\
& X_{2}^{\prime}=X_{1}+\omega^{2} X_{2}+\omega X_{3} \\
& X_{3}^{\prime}=X_{1}+\omega X_{2}+\omega^{2} X_{3} .
\end{aligned}
$$

For later use, notice the three following features: (a) In the $X_{i}^{\prime}$ - basis, the generator $\mathcal{S}$ acts as $\left(X_{1}, X_{2}, X_{3}\right) \rightarrow$ $\left(X_{1},-X_{2},-X_{3}\right)$ and it is represented by a non diagonal matrix given by Eq. (5.14); this change can be explicitly checked by computing $\mathcal{S}: X_{i}^{\prime} \rightarrow \tilde{X}_{i}^{\prime}=\mathcal{S}_{i j} X_{j}^{\prime}$. (b) The analogue of the character relation (2.1) of $\mathbb{A}_{4}$ reads in the $\mathbb{Z}_{3}$ case as follows

$$
\mathbb{Z}_{3}: 3=(1)^{2}+\left(1^{\prime}\right)^{2}+\left(1^{\prime \prime}\right)^{2}
$$

showing that there are three kinds of one- dimensional representations which, by using characters, can be denoted like $\mathbf{1}=\mathbf{1}_{1}, \mathbf{1}^{\prime}=\mathbf{1}_{\omega}$ and $\mathbf{1}^{\prime \prime}=\mathbf{1}_{\bar{\omega}}$. Notice moreover that by breaking $\mathbb{A}_{4}$ down to the above $\mathbb{Z}_{3}$, the $\mathcal{S}$ generator is no 
TABLE VII. Lepton and right-handed neutrino superfields and their quantum numbers under $\mathbb{Z}_{3}$.

\begin{tabular}{lccccc}
\hline \hline Superfields & $L_{i}$ & $e^{c}$ & $\mu^{c}$ & $\tau^{c}$ & $N_{i}^{c}$ \\
\hline $\mathbb{Z}_{3}$ & $\mathbf{1}_{1} \oplus \mathbf{1}_{\omega} \oplus \mathbf{1}_{\bar{\omega}}$ & $\mathbf{1}_{\bar{\omega}}$ & $1_{\omega}$ & $1_{1}$ & $\mathbf{1}_{1} \oplus \mathbf{1}_{\omega} \oplus \mathbf{1}_{\bar{\omega}}$ \\
\hline \hline
\end{tabular}

TABLE VIII. Higgs and flavon superfields and their quantum numbers under $\mathbb{Z}_{3}$.

\begin{tabular}{lcccccc}
\hline \hline Superfields & $H_{u}$ & $H_{d}$ & $\Phi_{i}$ & $\Omega_{i}$ & $S$ & $\chi$ \\
\hline $\mathbb{Z}_{3}$ & $\mathbf{1}_{1}$ & $\mathbf{1}_{\omega}$ & $\mathbf{1}_{1} \oplus \mathbf{1}_{\omega} \oplus \mathbf{1}_{\bar{\omega}}$ & $\mathbf{1}_{1} \oplus \mathbf{1}_{\omega} \oplus \mathbf{1}_{\bar{\omega}}$ & $\mathbf{1}_{\bar{\omega}}$ & $1_{1}$ \\
\hline \hline
\end{tabular}

longer a conserved symmetry. So, the triplet $\mathbf{3}_{(-1,0)}$ should be imagined as $\mathbf{3}_{0}$ where we have kept only the character of $\mathcal{T}$; and then can be decomposed in terms of irreducible representations like

$$
\mathbf{3}_{0}=\mathbf{1}_{1} \oplus \mathbf{1}_{\omega} \oplus \mathbf{1}_{\bar{\omega}}
$$

since all irreducible representations of $\mathbb{Z}_{3}$ are one- dimensional. The broken part of the symmetry is given by $\mathbb{V}_{4}$. The new quantum numbers of the different superfields get replaced, after the breaking $\mathbb{A}_{4} \rightarrow \mathbb{Z}_{3}$, by the ones defined in Tables VII and VIII. As a check, the monomials $S \nu_{e}^{c} \nu_{\mu}^{c}$, $S \nu_{\tau}^{c} \nu_{\tau}^{c}, \nu_{e}^{c} \nu_{e}^{c} \Omega_{1}, \nu_{\mu}^{c} \nu_{\tau}^{c} \Omega_{1}, \nu_{\tau}^{c} \nu_{\tau}^{c} \Omega_{2}, \nu_{e}^{c} \nu_{\mu}^{c} \Omega_{2}, \nu_{\mu}^{c} \nu_{\mu}^{c} \Omega_{3}, \nu_{e}^{c} \nu_{\tau}^{c} \Omega_{3}$, $\nu_{e}^{c} \nu_{e}^{c} \chi$ and $\nu_{\mu}^{c} \nu_{\tau}^{c} \chi$ involved in Eq. (3.6) are invariant under discrete $\mathbb{Z}_{3}$.

\section{Vacua and unproblematic domain walls}

After the breaking of the discrete $\mathbb{A}_{4}$ down to $\mathbb{Z}_{3}$, the flavon vacua $\langle\Phi\rangle$ sit in four degenerate points $\varphi_{1}, \varphi_{2}, \varphi_{3}, \varphi_{4}$ in the flavon space $\mathfrak{F}$. As the quantum number of these vacua is given by $\mathbb{V}_{4}$; it follows that the induced domain walls (5.7) are characterized by the Klein group $\mathbb{V}_{4} \sim \mathbb{Z}_{2} \times \mathbb{Z}_{2}^{\prime}$. One of the two $\mathbb{Z}_{2}$ factors is generated by the previous $\mathcal{S}$ while the other $\mathbb{Z}_{2}^{\prime}$ is generated by $\mathcal{S}^{\prime}$ whose expressions are given by (5.14). Thus, in order to investigate the domain walls extending between the degenerate vacua of the model created by the spontaneous breaking of the non-Abelian $\mathbb{A}_{4}$ group down to $\mathbb{Z}_{3}$, it is enough to use properties of its Abelian subgroups $\mathbb{Z}_{2}$ and $\mathbb{Z}_{3}$. Indeed, by thinking of $\mathbb{A}_{4}$ like $\mathbb{V}_{4} \rtimes \mathbb{Z}_{3}$, the breaking of the flavor symmetry driven by the flavon triplet $\Phi$ in the charged lepton sector may be expressed as

$$
\mathbb{V}_{4} \rtimes \mathbb{Z}_{3} \times \boldsymbol{Z}_{3} \stackrel{\langle\Phi\rangle}{\rightarrow} \mathbb{Z}_{3} \times Z_{3}
$$

So, the domain walls separating the $\varphi_{1}, \varphi_{2}, \varphi_{3}, \varphi_{4}$ flavon vacua can be completely characterized by the elements of $\mathbb{V}_{4} \simeq \mathbb{Z}_{2} \times \mathbb{Z}_{2}^{\prime}$. Being associated with $\mathbb{V}_{4}$, these vacua are then related to each other by $\mathbb{Z}_{2} \times \mathbb{Z}_{2}^{\prime}$ transformations as sketched on the following diagram

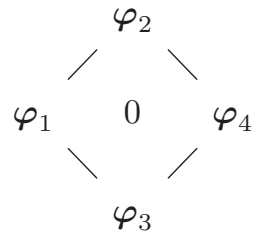

where the centre 0 refers to the singular situation where all four $\varphi_{a}$ 's vanish identically $-v_{\Phi} \rightarrow 0$. So, at this singular point lives the full $\mathbb{A}_{4}$ symmetry including its four $\mathbb{Z}_{3}$ subsymmetries. The $\varphi_{1}, \varphi_{2}, \varphi_{3}, \varphi_{4}$ have same ground state energy and can be obtained by acting on the $\mathbb{Z}_{3}$ - invariant vacuum (5.16) by the $\mathbb{V}_{4}$ generators $\mathcal{S}$ and $\mathcal{S}^{\prime}$. By taking $\varphi_{1}=\langle\Phi\rangle$ as in Eq. (5.16), we then have

$$
\varphi_{2}=\mathcal{S} \varphi_{1} ; \quad \varphi_{3}=\mathcal{S}^{\prime} \varphi_{1} ; \quad \varphi_{4}=\mathcal{S}^{\prime \prime} \varphi_{1}
$$

where we have set $\mathcal{S}^{\prime \prime}=\mathcal{S} \mathcal{S}^{\prime}=\mathcal{S}^{\prime} \mathcal{S}$. Notice that $\varphi_{4}$ can be also obtained from $\varphi_{2}$ or from $\varphi_{3}$; the relations are respectively given by $\varphi_{4}=\mathcal{S}^{\prime} \varphi_{2}$ and $\varphi_{4}=\mathcal{S} \varphi_{3}$. Using Eqs. (5.16) and (5.14), we can express the four vacua explicitly in $\mathbb{C}^{3}$ as follows

$$
\begin{aligned}
& \varphi_{1}=v_{\Phi}\left(\begin{array}{l}
1 \\
0 \\
0
\end{array}\right), \quad \varphi_{2}=\frac{v_{\Phi}}{3}\left(\begin{array}{c}
-1 \\
2 \\
2
\end{array}\right) \\
& \varphi_{3}=\frac{v_{\Phi}}{3}\left(\begin{array}{l}
-1 \\
2 \bar{\omega} \\
2 \omega
\end{array}\right), \quad \varphi_{4}=\frac{v_{\Phi}}{3}\left(\begin{array}{c}
-1 \\
2 \omega \\
2 \bar{\omega}
\end{array}\right)
\end{aligned}
$$

with $\omega=-\frac{1}{2}+i \frac{\sqrt{3}}{2}$. In the vector basis (5.4), we have to use real variables; so the dimension of the above vectors has to be doubled; for instance we have $\varphi_{4}^{T}=\frac{v_{\Phi}}{3}(-1,0,-1$, $\sqrt{3},-1,-\sqrt{3})$.

Observe that the four $\varphi_{a}$ vacua define four particular vectors in the complex three- (real six-) dimensional space $\mathbb{C}^{3} \sim \mathbb{R}^{6}$ of possible vacua of the $\Phi$ - flavon. As these vectors are constrained like

$$
\varphi_{1}+\varphi_{2}+\varphi_{3}+\varphi_{4}=0
$$

they define a tetrahedron with Kahler modulus given by $\operatorname{Re} v_{\Phi}$. By using the $(X, Y, Z)$ basis vectors; we can express the four $\varphi_{a}$ 's like $1 \oplus 3$, the sum of a singlet and a triplet with respect to the generator $\mathcal{T}$. The singlet is given by

$$
\varphi_{1}=v_{\Phi} X, \quad \mathcal{T} \varphi_{1}=\varphi_{1}
$$

and the remaining three others form a 3-cycle $\left(\varphi_{2}, \varphi_{3}, \varphi_{4}\right)$ with components as follows 


$$
\begin{aligned}
& \varphi_{2}=\frac{v_{\Phi}}{3}(-X+2 Y+2 Z) \\
& \varphi_{3}=\frac{v_{\Phi}}{3}(-X+2 \bar{\omega} Y+2 \omega Z) \\
& \varphi_{4}=\frac{v_{\Phi}}{3}(-X+2 \omega Y+2 \bar{\omega} Z) .
\end{aligned}
$$

Using the expression of $\mathcal{T}$ given by Eq. (5.14), one can easily check that they obey indeed the cyclic property

$$
\mathcal{T} \varphi_{2}=\varphi_{3}, \quad \mathcal{T} \varphi_{3}=\varphi_{4}, \quad \mathcal{T} \varphi_{4}=\varphi_{2}
$$

From the above relations, we learn that we indeed have the remarkable identity $\sum_{i} \varphi_{i}=0$ that leads in turns to $\varphi_{1}=-\left(\varphi_{2}+\varphi_{3}+\varphi_{4}\right)$. Observe also that for a fixed value of $v_{\Phi}$, the four $\varphi_{1}, \varphi_{2}, \varphi_{3}, \varphi_{4}$ are given by four special points in the three dimensional $\mathfrak{F} \simeq \mathbb{C}^{3}$. These points define the four vertices of a homogeneous tetrahedron given by the Fig. 9. Thus, from the view of the space of the flavon $\Phi$, the domain walls separating the four vacua (i.e., vertices) are encoded in a nonregular $3 \mathrm{~d}$ geometry having: $(\alpha)$ six 1- dimensional edges that we denote like $\varphi_{a b}=\left(\varphi_{a}, \varphi_{b}\right)$ with domain walls given by (5.7) namely

$$
l_{a b}= \pm \int_{\varphi_{a b}} \varpi_{1}
$$

with Hermitian 1-form $\varpi_{1}=\varpi_{1}\left(\xi_{\Phi}, \bar{\xi}_{\Phi}\right)$ given by

$$
\varpi_{1}=\frac{d \varphi}{\sqrt{\mathcal{E}+\tilde{\mathcal{V}}\left(\xi_{\Phi}, \bar{\xi}_{\Phi}\right)}}, \quad d \varphi^{2}=\sum_{i=1}^{3} d \bar{\xi}_{\Phi_{i}} d \xi_{\Phi_{i}}
$$

$(\beta)$ four triangular surfaces $\boldsymbol{T}_{a b c}$ with vertices $\left(\varphi_{a}, \varphi_{b}, \varphi_{c}\right)$, one of them is given by $\mathbf{T}_{234}$-the face with yellow color in Fig. 9. In this case, the domain walls extending between $\left(\varphi_{a}, \varphi_{b}, \varphi_{c}\right)$ are given by

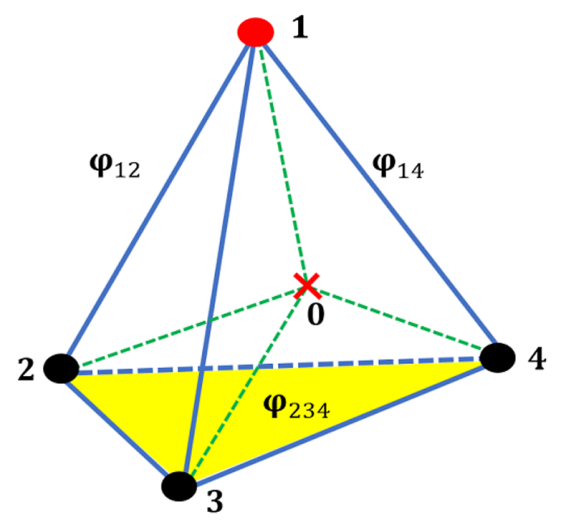

FIG. 9. Four vacua $\varphi_{a}$ related to each other by $\mathbb{V}_{4}$ transformations. They define the four vertices of a tetrahedron with $\mathbb{Z}_{3}$ axes $O \varphi_{a}$. It is interpreted here as the DWs quiver in the flavon space $\mathfrak{F}$. Dashed green lines represent the four $\mathbb{Z}_{3}$ subsymmetry axes of $\mathbb{A}_{4}$.

$$
S_{a b c}= \pm \int_{T_{a b c}} \varpi_{2}
$$

where $\varpi_{2}=\varpi_{2}\left(\xi_{\Phi}, \bar{\xi}_{\Phi}\right)$-generalizes the above 1- form $\varpi_{1}$-is a Hermitian 2 -form living on $\mathfrak{F}$ and dependent as well on the flavon scalar potential $\tilde{\mathcal{V}}\left(\xi_{\Phi}, \bar{\xi}_{\Phi}\right)$. $(\gamma)$ the bulk of the tetrahedron $\boldsymbol{H}_{1234}$ with vertices $\left(\varphi_{1}, \varphi_{2}, \varphi_{3}, \varphi_{4}\right)$ leading to

$$
\boldsymbol{V}_{1234}= \pm \int_{\boldsymbol{H}_{1234}} \varpi_{3}
$$

where now $\varpi_{3}=\varpi_{3}\left(\xi_{\Phi}, \bar{\xi}_{\Phi}\right)$ is a Hermitian 3-form on $\mathfrak{F}$. Notice that for the limit $v_{\Phi} \rightarrow 0$, all four $\varphi_{1}, \varphi_{2}, \varphi_{3}, \varphi_{4}$ vacua merge at the origin $O$ of $\mathfrak{F}$-the center of the tetrahedron-where lives the full $\mathbb{A}_{4}$ symmetry. In this singular limit, the DWs disappear. Notice also that on the $O \varphi_{1}$ axis normal to $\boldsymbol{T}_{234}$ face of the tetrahedron; it lives a $\mathbb{Z}_{3}$ subsymmetry of $\mathbb{A}_{4}$. In fact, on each of the four $O \varphi_{a}$ axes, passing through the center $O$ and the vertices $\varphi_{a}$, lives one of the four $\mathbb{Z}_{3}$ subsymmetries (2.3) of the discrete $\mathbb{A}_{4}$ characterizing the flavored NMSSM. Notice moreover that from Eq. (5.27) we learn that one can also use the elements $\left\{I, \mathcal{S}, \mathcal{S}^{\prime}, \mathcal{S S}^{\prime}\right\}$ of the $\mathbb{V}_{4}$ to describe the four degenerate vacua (5.29) and then the quivers representing the DWs. Indeed, seen that the Klein $\mathbb{V}_{4}$ is isomorphic to $\mathbb{Z}_{2} \times \mathbb{Z}_{2}$, the set $\left\{I, \mathcal{S}, \mathcal{S}^{\prime}, \mathcal{S S}^{\prime}\right\}$ can be then factorized in three manners as follows

$$
\begin{aligned}
& \mathbb{Z}_{2} \times \mathbb{Z}_{2}^{\prime}=\{I, \mathcal{S}\} \times\left\{I, \mathcal{S}^{\prime}\right\} \\
& \mathbb{Z}_{2} \times \mathbb{Z}_{2}^{\prime \prime}=\{I, \mathcal{S}\} \times\left\{I, \mathcal{S}^{\prime \prime}\right\} \\
& \mathbb{Z}_{2}^{\prime} \times \mathbb{Z}_{2}^{\prime \prime}=\left\{I, \mathcal{S}^{\prime}\right\} \times\left\{I, \mathcal{S}^{\prime \prime}\right\}
\end{aligned}
$$

with $\mathcal{S}^{\prime \prime}=\mathcal{S S}^{\prime}$ and $\mathcal{S}^{2}=\left(\mathcal{S}^{\prime}\right)^{2}=\left(\mathcal{S}^{\prime \prime}\right)^{2}=I$. Thus, we can represent the domain walls extending between the vacua by a real quiver diagram similar to Fig. 9; but with vertices given by the group elements of $\mathbb{V}_{4}=\left\{I, \mathcal{S}, \mathcal{S}^{\prime}, \mathcal{S S}^{\prime}\right\}$. In this case, the $\mathbb{Z}_{2}$ subgroups of $\mathbb{V}_{4}$ are associated with edges of the tetrahedron with a boundary point given by $I$ and the other boundary by one of the three other vertices. The four $\mathbb{Z}_{3}$ subsymmetries are associated with the four faces $\boldsymbol{T}_{a b c}$ of the diamond. Indeed, we can write down an explicit correspondence between the vacua $\varphi_{a}$ and the elements of $\mathbb{V}_{4}$. For example, for the three $\varphi_{1 a}$ edges, we have the following

$$
\begin{aligned}
(I, \mathcal{S}) & \leftrightarrow \varphi_{12} \\
\left(I, \mathcal{S}^{\prime}\right) & \leftrightarrow \varphi_{13} \\
\left(I, \mathcal{S}^{\prime \prime}\right) & \leftrightarrow \varphi_{14}
\end{aligned}
$$

By using (5.28), the vertex $\varphi_{1}$ is represented by the identity $I$ and the vertex $\varphi_{2}$ by the generator $\mathcal{S}$. A similar thing is valid for the other $\varphi_{13}$ and $\varphi_{14}$ edges with one vertex given 
by $\varphi_{1}$ and the other by $\varphi_{3}$ or $\varphi_{4}$. The three other remaining edges $\varphi_{23}, \varphi_{24}$, and $\varphi_{34}$ can be interpreted as follows

$$
\begin{aligned}
\varphi_{23} \leftrightarrow\left(\mathcal{S}, \mathcal{S}^{\prime}\right)=\mathcal{S} \times\left(I, \mathcal{S}^{\prime \prime}\right) \leftrightarrow \mathcal{S} \varphi_{14} \\
\varphi_{24} \leftrightarrow\left(\mathcal{S}, \mathcal{S}^{\prime \prime}\right)=\mathcal{S} \times\left(I, \mathcal{S}^{\prime}\right) \leftrightarrow \mathcal{S} \varphi_{13} \\
\varphi_{34} \leftrightarrow\left(\mathcal{S}^{\prime}, \mathcal{S}^{\prime \prime}\right)=\mathcal{S}^{\prime} \times(I, \mathcal{S}) \leftrightarrow \mathcal{S}^{\prime} \varphi_{12}
\end{aligned}
$$

where for instance, $\varphi_{23}$ is just the transformation of $\varphi_{14}$ under $\mathcal{S}$. Notice also that the face $\boldsymbol{T}_{234}$ is associated with $\left(\mathcal{S}, \mathcal{S}^{\prime}, \mathcal{S}^{\prime \prime}\right)$ and is related with the $\mathbb{Z}_{3}$ subsymmetry of $\mathbb{A}_{4}$; so

$$
\boldsymbol{T}_{234} \leftrightarrow \mathbb{Z}_{3}=\left\{I, \mathcal{T}, \mathcal{T}^{2}\right\}
$$

with $\mathcal{T}=(234)$. The domain walls induced by the breaking $\mathbb{A}_{4} \rightarrow \mathbb{Z}_{3}$ may be also described by using characters of the Klein four- group $\mathbb{V}_{4}$ as in Fig. 10. These characters are given by $(+,+),(-,+),(+,-),(-,-)$ respectively associated with the group elements $I, \mathcal{S}, \mathcal{S}^{\prime}, \mathcal{S} \mathcal{S}^{\prime}$. After this graphic description of DWs in the VEV space $\mathfrak{F}$, we are now in position to address the question on whether these domain walls of the FNMSSM are problematic in the charged lepton sector or not. The answer is fortunately no!; this is due to the very high energy scale where the spontaneous breaking of the discrete group $\mathbb{A}_{4} \rightarrow \mathbb{Z}_{3}$ takes place. In this regard, recall that the inflation based scenario might be a nice approach to bypass DWs provided the inflationary scale; say around $\mathcal{O}\left(10^{14}\right) \mathrm{GeV}[110,111]$, is lower than the flavor symmetry breaking scale. In this case, the DWs are formed before the end of inflation at scales comparable to the GUT scale [110]. Hence, assuming that the cutoff scale $\Lambda$ associated to $\mathbb{A}_{4}$ breaking given in Eq. (2.12) is of order of the SUSY-GUT scale $M_{\mathrm{GUT}} \sim 2 \times 10^{16} \mathrm{GeV}$, we find by using (2.12) the following lower bound of the flavon VEV

$$
v_{\Phi}>1.4 \times 10^{14} \mathrm{GeV}
$$

Therefore, the flavon triplet $\Phi$ acquired its VEV before the end of inflation; thus, in the charged lepton sector the discrete $\mathbb{A}_{4}$ symmetry is broken at a scale that is above the

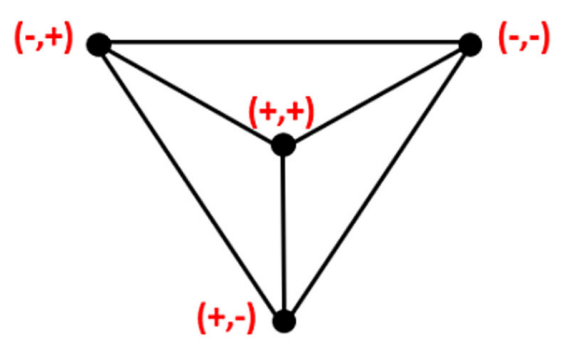

FIG. 10. A quiver diagram representing domain walls using characters of the generators of the Klein group. The four vertices correspond to the four degenerate vacua, while the edges that connects the four vertices stand for domain walls. inflationary scale. Consequently, the domain walls produced in this case are inflated away; and one is left with the $\mathbb{Z}_{3}$ invariant ground state $\varphi_{1}$.

\section{Domain walls in neutrino sector}

In this subsection we study the domain walls in the chargeless lepton sector of the flavored NMSSM and show that they are really problematic. Recall that in the neutrino sector, the breaking pattern corresponds to $\mathbb{A}_{4} \rightarrow \mathbb{Z}_{2}$; and can be driven either by the VEV of the flavon $\Omega$; or by the VEV of the singlet $S$. This feature may be observed from the properties of the quantum numbers given by Table III. So, we first study the breaking $\mathbb{A}_{4} \rightarrow \mathbb{Z}_{2}$ by using a diagonal VEV in case of the flavon $\Omega$; then we make comments on the breaking using $S$.

\section{Breaking $\mathbb{A}_{4}$ to $\mathbb{Z}_{2}$ by flavon $\Omega$ and stable domain walls}

By using the flavon $\Omega$, the breaking $\mathbb{A}_{4} \rightarrow \mathbb{Z}_{2}$ is realised by Eq. (5.18). Clearly, this particular flavon triplet $\Omega$ vacuum aligned along the diagonal of the complex space $\mathfrak{F} \simeq \mathbb{C}^{3}$ is not a symmetry of $\mathcal{T}$; but preserves the $\mathcal{S}$ generator,

$$
\mathcal{S}\langle\Omega\rangle=\langle\Omega\rangle, \quad \mathcal{T}\langle\Omega\rangle \neq\langle\Omega\rangle .
$$

These relations can be explicitly checked by using the matrix representations of $\mathcal{T}$ and $\mathcal{S}$ given by Eq. (5.14). As a consequence of this breaking of $\mathbb{A}_{4}$ down to $\mathbb{Z}_{2}=\{I, \mathcal{S}\}$; the quantum charges of the right-handed neutrino $N_{i}^{c}$ and the lepton doublets $L_{i}$ superfields under the $\mathbb{Z}_{2}$ residual symmetry are summarized in Tables IX and $X$. Therefore, we have the following symmetry transformations

$$
\begin{array}{rlrl}
\nu_{e}^{c} & \rightarrow-\nu_{e}^{c}, & \nu_{\mu}^{c} \rightarrow-\nu_{\mu}^{c}, & \nu_{\tau}^{c} \rightarrow-\nu_{\tau}^{c} \\
L_{e} \rightarrow-L_{e}, & L_{\mu} \rightarrow-L_{\mu}, & L_{\tau} \rightarrow-L_{\tau}
\end{array}
$$

while the right-handed charged leptons are $\mathbb{Z}_{2}$ even, because the singlet representations $\left(\mathbf{1}_{(1,1)}, \mathbf{1}_{(1, \omega)}, \mathbf{1}_{\left(1, \omega^{2}\right)}\right)$ transform trivially under $\mathcal{S}$, see Eq. (A2) of Appendix A.

By substituting $\mathbb{A}_{4}$ by $\mathbb{V}_{4} \rtimes \mathbb{Z}_{3}$ and the four order group $\mathbb{V}_{4}$ by $\mathbb{Z}_{2} \times \mathbb{Z}_{2}^{\prime}$, the breaking pattern $\mathbb{A}_{4} \rightarrow \mathbb{Z}_{2}$ can be expressed like

$$
\left(\mathbb{Z}_{2} \times \mathbb{Z}_{2}^{\prime}\right) \rtimes \mathbb{Z}_{3} \stackrel{\langle\Omega\rangle}{\rightarrow} \mathbb{Z}_{2}
$$

TABLE IX. Lepton and right-handed neutrino superfields and their quantum numbers under $\mathbb{Z}_{2}$.

\begin{tabular}{lcc}
\hline \hline Superfields & $L_{i}$ & $N_{i}^{c}$ \\
\hline $\mathbb{Z}_{2}$ & $\mathbf{1}_{-} \oplus \mathbf{1}_{-} \oplus \mathbf{1}_{-}$ & $\mathbf{1}_{-} \oplus \mathbf{1}_{-} \oplus \mathbf{1}_{-}$ \\
\hline \hline
\end{tabular}


TABLE X. Higgs and flavon superfields and their quantum numbers under $\mathbb{Z}_{2}$.

\begin{tabular}{lcccccc}
\hline \hline Superfields & $H_{u}$ & $H_{d}$ & $\Phi$ & $\Omega$ & $S$ & $\chi$ \\
\hline $\mathbb{Z}_{2}$ & $\mathbf{1}_{+}$ & $\mathbf{1}_{+}$ & $\mathbf{1}_{-} \oplus \mathbf{1}_{-} \oplus \mathbf{1}_{-}$ & $\mathbf{1}_{+} \oplus \mathbf{1}_{+} \oplus \mathbf{1}_{+}$ & $\mathbf{1}_{+}$ & $\mathbf{1}_{+}$ \\
\hline \hline
\end{tabular}

This breaking mode shows that the induced domain walls are characterized by the broken part of the alternating $\mathbb{A}_{4}$ flavor symmetry which may naively be presented as $\mathbb{Z}_{2}^{\prime} \rtimes \mathbb{Z}_{3}$. The $\mathbb{Z}_{2}$ and $\mathbb{Z}_{2}^{\prime}$ groups are subgroups of the Klein four- group $\mathbb{V}_{4}$ respectively generated by $\mathcal{S}$ and $\mathcal{S}^{\prime}=\mathcal{T} \mathcal{S T}^{-1}$. The $\mathbb{Z}_{3}$ is generated by $\mathcal{T}$, it is one of the four kinds of $\mathbb{Z}_{3}$ subgroups inside of $\mathbb{A}_{4}$. Under the breaking (5.45), the initial $\mathbb{A}_{4}$ invariant vacuum gets now split into six vacua with same energy

$$
\langle\Omega\rangle_{i} \equiv \vartheta_{i}, \quad i=1, \ldots, 6
$$

In the space $\mathfrak{F}$ of flavons, these vacua define the six vertices of a homogeneous octahedron $\sum_{i=1}^{6} \vartheta_{i}=0$ embedded in $\mathbb{R}^{6} \sim \mathbb{C}^{3}$ as schematized in Fig. 11. Notice that one of the six vacua (5.46), say $\vartheta_{1}$, is just the $\mathbb{Z}_{2}$ - invariant vacuum Eq. (5.18); that is $\vartheta_{1}=\langle\Omega\rangle$. By using the basis vectors $X$, $Y, Z$, it can be expressed as follows

$$
\vartheta_{1}=v_{\Omega}(X+Y+Z)
$$

and points in the diagonal direction of the complex $3 \mathrm{~d}$ (real 6-) space. To get the remaining five other $\vartheta_{i}$ vacua, we act on this $\vartheta_{1}$ by the $\mathcal{S}^{\prime}$ and $\mathcal{T}$ generators of $\mathbb{Z}_{2}^{\prime} \rtimes \mathbb{Z}_{3}$. As the $\mathcal{S}^{\prime}$ acts on $\vartheta_{1}$ like a reflection: $\vartheta_{1} \rightarrow-\vartheta_{1}$, it is instructive to denote the six $\vartheta_{i}$ vacua like $\vartheta_{\alpha}^{ \pm}$with $\alpha=1,2,3$. Using the explicit expression of $\mathcal{S}^{\prime}$ and $\mathcal{T}$, we obtain

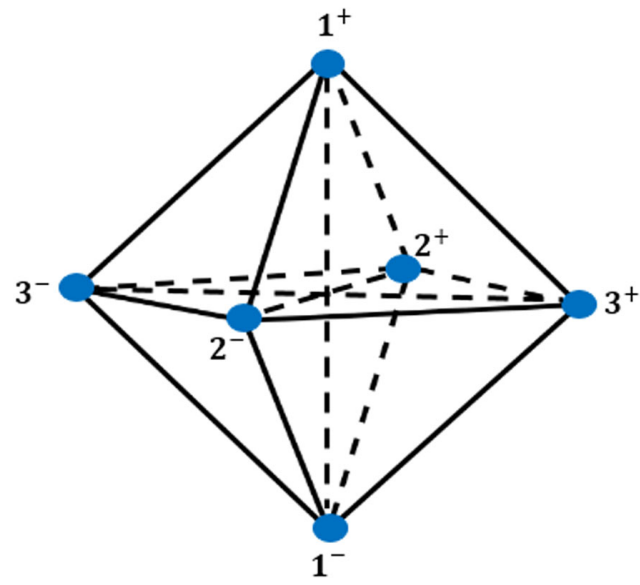

FIG. 11. The six vacua define the vertices of a homogeneous octahedron of Kahler modulus $v_{\Omega}$. While the edges stand for the domain walls interpolating between these six degenerate vacua.

$$
\begin{aligned}
& \vartheta_{1}^{ \pm}= \pm v_{\Omega}(X+Y+Z) \\
& \vartheta_{2}^{ \pm}= \pm v_{\Omega}(X+\bar{\omega} Y+\omega Z) \\
& \vartheta_{3}^{ \pm}= \pm v_{\Omega}(X+\omega Y+\bar{\omega} Z)
\end{aligned}
$$

with

$$
\mathcal{S}\left\langle\vartheta_{\alpha}^{ \pm}\right\rangle=\left\langle\vartheta_{\alpha}^{ \pm}\right\rangle
$$

Explicitly, we have

$\vartheta_{1}^{ \pm}= \pm v_{\Omega}\left(\begin{array}{c}1 \\ 1 \\ 1\end{array}\right), \quad \vartheta_{2}^{ \pm}= \pm v_{\Omega}\left(\begin{array}{c}1 \\ \bar{\omega} \\ \omega\end{array}\right), \quad \vartheta_{3}^{ \pm}= \pm v_{\Omega}\left(\begin{array}{c}1 \\ \omega \\ \bar{\omega}\end{array}\right)$

with the transformation relations

$$
\begin{aligned}
& \vartheta_{1}^{ \pm}, \quad \vartheta_{2}^{ \pm}=\mathcal{T} \vartheta_{1}^{ \pm}, \quad \vartheta_{3}^{ \pm}=\mathcal{T} \vartheta_{2}^{ \pm}, \\
& \mathcal{S}^{\prime} \vartheta_{2}^{ \pm}=\vartheta_{2}^{ \pm}, \quad \mathcal{S}^{\prime} \vartheta_{1 / 3}^{ \pm}=\vartheta_{1 / 3}^{\mp}
\end{aligned}
$$

with $\mathcal{S}^{\prime}=\mathcal{T S T ^ { - 1 }}$. Notice the real VEV $v_{\Omega}$ of the chiral superfield $\Omega$, which breaks $\mathbb{A}_{4}$ as in (5.45), breaks as well the extra $\boldsymbol{Z}_{3}$ symmetry. ${ }^{10}$ Notice also that contrary to the charged sector, domain walls induced by the breaking pattern $\mathbb{A}_{4} \rightarrow \mathbb{Z}_{2}$ in the neutrino sector are problematic. The couplings involved in the superpotential (3.5) are at the renormalizable level where we expect a particular hierarchy among the VEVs as $v_{S}<v_{\chi} \lesssim v_{\Omega}$. This hierarchy is reasonable since $v_{S}$ is known to acquire a VEV of the order of $M_{\text {SUSY }}$ and it is also responsible for a small deviation form the TBM pattern and thus should be smaller than the other VEVs while the VEVs of the flavons $\chi$ and $\Omega$ can be safely assumed to vary in the range $\left[10^{7} \rightarrow 10^{10}\right] \mathrm{GeV}$ which will lead to right-handed neutrino masses $M_{i}$ in Eq. (3.19) compatible with type-I seesaw mechanism. This can be accomplished simply by choosing the right values for the free parameters $Y_{0}, \lambda, \lambda^{\prime}$ and $\beta$ involved in $v_{u}=v \sin \beta$. Therefore, these VEVs are then smaller than the inflationary scale and thus, the domain walls in the neutrino sector of the flavored NMSSM are created below the inflationary scale; they are inevitable and then inconsistent with the standard cosmology [112]; they must be avoided. In order to circumvent this domain wall problem, we break explicitly the $\mathbb{Z}_{2}^{\prime} \rtimes \mathbb{Z}_{3}$ permuting the six degenerate vacua. This will be done by using higher order Planck-suppressed operators $\mathcal{O}\left(\frac{1}{M_{\mathrm{Pl}}}\right)$.

\footnotetext{
${ }^{10}$ Notice that the domain walls created by this extra $Z_{3}$ symmetry during the breaking pattern Eq. (5.45) in the neutrino sector will disappear together with the ones from the broken $\mathbb{A}_{4}$.
} 


\section{Solving the DW problem in neutrino sector}

To start, recall that several ways have been suggested in the literature to overcome the domain wall problem induced by breaking discrete symmetries of the degenerate vacua; the simplest one being inflation [18]. A second interesting method to solve the domain wall problem is that the discrete symmetry connecting the vacua must be broken explicitly before the spontaneous breaking takes place [113]. It was shown in Refs. [20,114-118] that the last method can be achieved by introducing higher dimensional operators $\frac{1}{M_{\mathrm{Pl}}^{n}} \mathcal{O}_{n+3}$ suppressed by powers of the Planck scale $M_{\mathrm{Pl}}$ leading to favor one of the vacua over the others, and consequently no walls will be formed and the domain wall problem is resolved; for NMSSM see [75]. Moreover, an analysis has been performed in Refs. $[119,120]$ proves that the relevant non-renormalizable operators $\mathcal{O}_{n+3}$ that solve the problem are the odd superpotential terms ( $\mathrm{n}$ even integer). However, we should notice that there are also dimensional operators of order four (operators suppressed by one inverse power of the Planck mass) that must be eliminated. We do not go into details here but we point out that this can be realized by invoking an additional symmetry such as extra $\mathcal{Z}_{2}$ or $\mathcal{Z}_{5} \mathrm{R}$-symmetries; for details in this direction see for instance Refs. [119,120]. In the present paper, we follow Ref. [121] that adopts the second approach; concretely we break the $\mathbb{Z}_{2}^{\prime} \rtimes \mathbb{Z}_{3} \times \boldsymbol{Z}_{3}$ part of the flavor symmetry explicitly via higher dimensional operators-Planck scale operators-of order five; in other words, we want to keep only one vacuum among the six $\vartheta_{\alpha}^{ \pm}$; for instance $\vartheta_{1}^{+}$. For our concern, the $\mathbb{A}_{4} \times \boldsymbol{Z}_{3}$ invariant superpotential $W_{\text {scal }}$ restricted to the Higgs doublet $H_{u, d}$, the gauge singlet $S$ and the flavon superfields $\chi, \Omega, \Phi$ is as follows

$$
\begin{aligned}
W_{\text {scal }}= & \lambda_{1} S H_{u} H_{d}+\lambda_{2} S^{3}+\lambda_{3} \Omega^{3}+\lambda_{4} \Phi^{3}+\lambda_{5} \chi^{3} \\
& +\lambda_{6} S \Omega^{2}+\lambda_{7} \chi \Omega^{2}+\mu_{\Phi} \Phi^{2}
\end{aligned}
$$

The non-renormalizable operators $\mathcal{W}_{\mathrm{NR}}$ of order five operators-suppressed by two inverse powers of the Planck mass- that break the full discrete symmetry group of our model down to $\mathbb{Z}_{2}$ can be chosen as given by a perturbation $\delta W_{\text {scal }}$ of one of the trilinear couplings that already exist in the scalar superpotential (5.52). Thus, we are left with multiple choices for order five operators where the most suitable one that can break explicitly $\mathbb{A}_{4} \times \boldsymbol{Z}_{3}$ down to $\mathbb{Z}_{2}$ is given by the following operator

$$
\mathcal{W}_{\mathrm{NR}}=\left.\frac{\lambda_{3}^{\prime}}{M_{\mathrm{Pl}}^{2}}\left(\Omega^{5}\right)\right|_{(1, \omega)}
$$

and, at quantum level, is represented by the Feynman diagram in Fig. 12 contributing to the effective potential $\mathcal{V}_{\text {eff }}$. Higher dimensional operators of order five that can

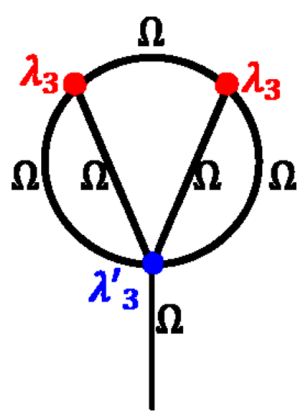

FIG. 12. The tadpole diagram for the nonrenormalizable term in Eq. (5.53).

contribute to the explicit breaking of the full flavor group are shown in Appendix C.

The choice of above $\mathcal{W}_{\mathrm{NR}}$ has been motivated by the fact that $\Omega$ carries quantum charges under both $\mathbb{A}_{4}$ and $Z_{3}$ symmetries. ${ }^{11}$ Moreover, this triplet creates its own walls during the spontaneous breaking of the full discrete symmetry $G_{\mathrm{f}}$. For the breaking of $\mathrm{A}_{4}$, the DWs are located on the boundaries of the six degenerate vacua given in Eq. (5.50) and Fig. 11. We note that the other fivedimensional operators (given in Appendix C) do not present any risk on the phenomenology of the model, since their contributions at low energy theory are generated through the same tadpole diagram presented in Fig. 12 with a factor difference depending on the coupling constant used in the perturbation term. By using the Feynman rules of supergraphs $^{12}$ [122-124], we obtain the following contribution to the effective scalar potential

$$
\delta \mathcal{V}_{\mathrm{eff}}=M_{W}^{3}\left(\eta \phi_{\Omega}+\bar{\eta} \bar{\phi}_{\Omega}\right)+M_{W}^{2}\left(\eta \mathrm{F}_{\Omega}+\bar{\eta} \overline{\mathrm{F}}_{\Omega}\right)
$$

where $\phi_{\Omega}$ and $\mathrm{F}_{\Omega}$ are respectively the scalar component and the F-term of the flavon superfield $\Omega=\phi_{\Omega}+\theta \psi_{\Omega}+\theta^{2} \mathrm{~F}_{\Omega}$; the $M_{W}$ is the scale of the electroweak theory and $\eta=\frac{\lambda_{3}^{2} \lambda_{3}^{\prime}}{\left(16 \pi^{2}\right)^{3}}$. It is clear that this operator induces a linear term in the soft SUSY breaking scalar potential-the full soft SUSY breaking terms are given by Eq. (B2) of Appendix B-expressed as

$$
\mathcal{V}_{\text {soft }} \supset \eta M_{W}^{3} \phi_{\Omega}+\text { H.c }
$$

and since the triplet $\phi_{\Omega}$ transforms nontrivially under $\mathbb{A}_{4} \times \boldsymbol{Z}_{3}$ flavor symmetry, the term in Eq. (5.55) breaks explicitly $\mathbb{A}_{4} \times \boldsymbol{Z}_{3}$ down to $\mathbb{Z}_{2}$. This contribution creates an energy gap among the degenerate vacua of $\Omega$ (5.50) with

\footnotetext{
${ }^{11}$ It is clear that the nonrenormalizable operator given in Eq. (5.53) will have negligible effect on the low energy phenomenology, considering the fact that this operator is suppressed by two power of the Planck mass.

${ }^{12}$ For the detailed calculations that led to the term given in the effective potential $\delta \mathcal{V}_{\text {eff }}$, see the analysis given in Appendix C.
} 
energetically dominant vacuum given by the $\vartheta_{1}^{+}$of (5.50) whose region in space start to expand and thus pushing the walls away.

\section{SUMMARY AND CONCLUSION}

Motivated by the recent progress in describing neutrino masses and mixing consistent with current data by using non-Abelian discrete flavor symmetries as well as their possible implications in cosmology, we have explored in this paper the neutrino phenomenology in a flavored NMSSM based on the flavor symmetry $G_{\mathrm{f}}=\mathbb{A}_{4} \times Z_{3}$ and studied the domain wall problem related to the spontaneous breaking of $G_{\mathrm{f}}$. To perform this study, we have first studied the extension of the usual NMSSM with $G_{f}$ and three right-handed neutrino in order to engineer appropriate neutrino masses and mixing. Then, we have analyzed the phenomenological implications of this FNMSSM including the problem of domain walls and provided a solution to this issue by using the breaking of the flavour symmetry and the effective scalar potential approach.

In our modeling, the non-Abelian alternating $\mathbb{A}_{4}$ group has been motivated by its simplest and its straightforwardness to describe the $\mu-\tau$ symmetry, while the extra $Z_{3}$ discrete symmetry plays several roles such as avoiding the communication between the charged lepton and neutrino sectors, solving the usual $\mu$-problem of the MSSM and eliminating unwanted terms in the superpotential. This FNMSSM is also motivated by the several implications carried by the NMSSM singlet superfield $S$ where in addition to its role of dynamically generating the $\mu$-term and its contribution to the Higgs mass, here it also contributes to the neutrino masses.

Indeed, the first main objective of this paper is to study the neutrino masses and mixing and their phenomenological implications. First, we showed that the light neutrino mass matrix of the TBM type arises at the renormalizable level through the type I seesaw mechanism; this is achieved by using the $\mathbb{A}_{4}$ representation properties and only one $\mathbb{A}_{4}$ flavon triplet $\Omega$ where we made use of the flavon singlet $\chi$ in the Majorana neutrinos mass matrix. Then, in order to generate neutrino masses and mixing compatible with the experimental data, a Majorana term arises in the superpotential from the contribution of the singlet $S$ plays the role of a deviation from the TBM pattern. The adopted deviation leads to the well-known trimaximal mixing predicting nonzero reactor angle $\theta_{13}$ as well as a near maximal atmospheric angle $\theta_{23}$ is permitted. Next, we studied the phenomenological implications of the neutrino sector where by using the $3 \sigma$ allowed ranges of the oscillation parameters, we showed through scatter plots the allowed ranges for the sum of neutrino masses $\sum m_{i}$, the effective Majorana neutrino mass $m_{e e}$, the effective electron neutrino mass $m_{\beta}$, and the Dirac $C P V$ phase $\delta_{C P}$. This study is done for both mass hierarchies where we found that numerical results concerning the ranges of these parameters may be tested in current and future experiments with a slight preference for the normal hierarchy over the inverted one. We report in the following Table a summary of the obtained ranges.

\begin{tabular}{ll}
\hline Normal Hierarchy & \multicolumn{1}{c}{ Inverted Hierarchy } \\
\hline $0.007271 \lesssim m_{e e}(\mathrm{eV}) \lesssim 0.04202$ & $0.05519 \lesssim m_{e e}(\mathrm{eV}) \lesssim 0.0641$ \\
$0.00959 \lesssim m_{\nu_{e}}(\mathrm{eV}) \lesssim 0.0439$ & $0.0554 \lesssim m_{\nu_{e}}(\mathrm{eV}) \lesssim 0.0638$ \\
$0.063 \lesssim \sum m_{i}(\mathrm{eV}) \lesssim 0.153$ & $0.14 \lesssim \sum m_{i}(\mathrm{eV}) \lesssim 0.17$ \\
$0.0043 \lesssim m_{\text {lightest }}(\mathrm{eV}) \lesssim 0.043$ & $0.0282 \lesssim m_{\text {lightest }}(\mathrm{eV}) \lesssim 0.041$ \\
$0.87 \pi(\& 1.04 \pi) \lesssim \delta_{C P} \lesssim$ & $1.12 \pi \lesssim \delta_{C P} \lesssim 1.93 \pi$ \\
$0.96 \pi(\& 1.94 \pi)$ & \\
\hline
\end{tabular}

On a related note, lepton flavor violating decays generally gives strong constraints on off-diagonal couplings and scalar masses which might in turns have an impact on the phenomenology of our model; the discussion of these processes as well as the dangerous contribution to flavor changing neutral currents (FCNCs) are left as future work.

The second main goal of this paper concerns the domain wall problem induced by the spontaneous breaking of the $\mathbb{A}_{4} \times Z_{3}$ discrete flavour group. To discuss this issue, we first explained the different breaking patterns encountered in the charged leptons and neutrino sectors. In the former, the spontaneous breaking is generated by the flavon triplet $\Phi$ that leads to the formation of domain walls among the degenerate VEVs of $\Phi$ up to $\mathbb{Z}_{2} \times \mathbb{Z}_{2}$ transformations, however, we showed that these walls do not present any danger since this kind of breaking took place at the inflationary scale. This is not the case for the breaking pattern in the neutrino sector driven by the flavon triplet $\Omega$ and leading to the creation of its own domain walls up to $\mathbb{Z}_{2} \rtimes \mathbb{Z}_{3} \times \boldsymbol{Z}_{3}$ charges that are problematic since this breaking occurs far below the inflationary scale. To deal with this problem, we added a nonrenormalizable term to the superpotential-suppressed by two units of the Planck scale - that breaks explicitly the $\mathbb{Z}_{2} \rtimes \mathbb{Z}_{3} \times \boldsymbol{Z}_{3}$ discrete symmetry. Normally, there are too many choices for the nonrenormalizable term, and we have chosen one of them to develop its contribution to the effective potential in details while we reported the rest of the terms-which lead to a similar contribution to the one we discussed in details-in Appendix C.

\section{APPENDICES}

We give four appendices where we report some technical details.

\section{APPENDIX A: THE DISCRETE ALTERNATING $\mathbb{A}_{4}$ GROUP}

The alternating group $\mathbb{A}_{4}$ has two noncommuting generators $\mathcal{S}$ and $\mathcal{T}$ satisfying the following relation: 
$\mathcal{S}^{2}=\mathcal{T}^{3}=(\mathcal{S T})^{3}=I$. Because of their noncommutativity-ST $\neq \mathcal{T} \mathcal{S}$ - only one of these generators can be chosen diagonal. In the present paper we have worked in the Altarelli-Feruglio (AF) basis where the generator $\mathcal{T}$ is diagonal [63]. Recall that the discrete group $A_{4}$ has four irreducible representations; one irreducible triplet $\mathbf{3}_{(-1,0)}$ and three different singlets $\mathbf{1}_{(1,1)}, \mathbf{1}_{(1, \omega)}, \mathbf{1}_{(1, \bar{\omega})}$ distinguished by their representation characters. In the AF basis, the three-dimensional unitary representation of the generators $\mathcal{T}$ and $\mathcal{S}$ is given by

$$
\mathbf{3}_{(-1,0)}: \mathcal{S}=\frac{1}{3}\left(\begin{array}{ccc}
-1 & 2 & 2 \\
2 & -1 & 2 \\
2 & 2 & -1
\end{array}\right), \quad \mathcal{T}=\left(\begin{array}{ccc}
1 & 0 & 0 \\
0 & \bar{\omega} & 0 \\
0 & 0 & \omega
\end{array}\right)
$$

where $\omega=e^{\frac{2 \pi i}{3}}$, while on the one-dimensional representations, these generators are represented by

$$
\begin{aligned}
\mathbf{1}_{(1,1)}: \mathcal{S}=1, \mathcal{T}=1 ; \quad \mathbf{1}_{(1, \omega)}: \mathcal{S}=1, \mathcal{T}=\bar{\omega} \\
\mathbf{1}_{(1, \bar{\omega})}: \mathcal{S}=1, \mathcal{T}=\omega .
\end{aligned}
$$

On the other hand, the $\mathbb{A}_{4}$ representations which are denoted by their basis characters obey the following tensor product algebra

$$
\begin{aligned}
& \mathbf{3}_{(-1,0)} \times \mathbf{3}_{(-1,0)}=\mathbf{1}_{(1,1)}+\mathbf{1}_{(1, \omega)}+\mathbf{1}_{\left(1, \omega^{2}\right)}+\mathbf{3}_{(-1,0)}+\mathbf{3}_{(-1,0)} \\
& \mathbf{3}_{(-1,0)} \times \mathbf{1}_{\left(1, \omega^{r}\right)}=\mathbf{3}_{(-1,0)} \\
& \mathbf{1}_{\left(1, \omega^{r}\right)} \times \mathbf{1}_{\left(1, \omega^{s}\right)}=\mathbf{1}_{\left(1, \omega^{r+s}\right)}
\end{aligned}
$$

where $r$ and $s$ take values $0,1,2$. Here we give useful tools for the computation of the tensor product of $\mathbb{A}_{4}$ triplets. For the case of two $\mathbb{A}_{4}$ triplets taken as $\mathbf{a}=\left(a_{1}, a_{2}, a_{3}\right)$ and $\mathbf{b}=\left(b_{1}, b_{2}, b_{3}\right)$, their tensor product is reducible with irreducible components given by the first decomposition relation in Eq. (A3). These irreducible components are given in a $\mathcal{T}$-diagonal basis by

$$
\begin{aligned}
& \left.(\mathbf{a} \otimes \mathbf{b})\right|_{1}=a_{1} b_{1}+a_{2} b_{3}+a_{3} b_{2}, \\
& \left.(\mathbf{a} \otimes \mathbf{b})\right|_{1^{\prime}}=a_{3} b_{3}+a_{2} b_{1}+a_{1} b_{2} \\
& \left.(\mathbf{a} \otimes \mathbf{b})\right|_{1^{\prime \prime}}=a_{2} b_{2}+a_{1} b_{3}+a_{3} b_{1}
\end{aligned}
$$

and

$$
\begin{aligned}
& \left.(\mathbf{a} \otimes \mathbf{b})\right|_{3_{S}}=\frac{1}{3}\left(\begin{array}{l}
2 a_{1} b_{1}-a_{2} b_{3}-a_{3} b_{2} \\
2 a_{3} b_{3}-a_{2} b_{1}-a_{1} b_{2} \\
2 a_{2} b_{2}-a_{1} b_{3}-a_{3} b_{1}
\end{array}\right), \\
& \left.(\mathbf{a} \otimes \mathbf{b})\right|_{3_{A}}=\frac{1}{2}\left(\begin{array}{l}
a_{2} b_{3}-a_{3} b_{2} \\
a_{1} b_{2}-a_{2} b_{1} \\
a_{3} b_{1}-a_{1} b_{3}
\end{array}\right) .
\end{aligned}
$$

Recall that the $\mathbb{A}_{4}$ group has four conjugacy classes $\mathcal{C}_{1}, \mathcal{C}_{2}$, $\mathcal{C}_{3}, \mathcal{C}_{4}$ given by

$$
\begin{aligned}
& \mathcal{C}_{1}=\{e\}, \quad \mathcal{C}_{2}=\left\{\mathcal{S}, \mathcal{T} \mathcal{S} \mathcal{T}^{-1}, \mathcal{T}^{-1} \mathcal{S T}\right\} \\
& \mathcal{C}_{3}=\{\mathcal{T}, \mathcal{T} \mathcal{S}, \mathcal{S} \mathcal{T}, \mathcal{S} \mathcal{S}\}, \quad \mathcal{C}_{4}=\left\{\mathcal{T}^{2}, \mathcal{S} \mathcal{T}^{2}, \mathcal{T}^{2} \mathcal{S}, \mathcal{T} \mathcal{S} \mathcal{T}\right\}
\end{aligned}
$$

and they are used in building the character Table $\chi_{R_{i}}\left(\mathcal{C}_{j}\right)=$ $\chi_{i j}$ of the group $\mathbb{A}_{4}$ which reads as follows

\begin{tabular}{|l|l|l|l|l|}
\hline$\chi_{i j}\left(\mathbb{A}_{4}\right)$ & $\boldsymbol{R}_{1}$ & $\boldsymbol{R}_{1^{\prime}}$ & $\boldsymbol{R}_{1^{\prime \prime}}$ & $\boldsymbol{R}_{3}$ \\
\hline $\mathcal{C}_{1}$ & 1 & 1 & 1 & 3 \\
\hline $\mathcal{C}_{2}$ & 1 & 1 & 1 & -1 \\
\hline $\mathcal{C}_{3}$ & 1 & $\omega$ & $\omega^{2}$ & 0 \\
\hline $\mathcal{C}_{4}$ & 1 & $\omega^{2}$ & $\omega$ & 0 \\
\hline
\end{tabular}

To get these numbers, one uses the formula $\chi_{\varrho}(g)=\operatorname{tr}\left(\varrho_{g}\right)$ with $\varrho$ an irreducible representation and $g$ a group element. Because of the trace cyclicity property, this formula is invariant under group conjugation $h g h^{-1}$. For instance, we have for $\chi_{\boldsymbol{R}_{3}}(e)=\operatorname{tr}\left(I_{3}\right)=3$, and for $\chi_{\boldsymbol{R}_{3}}(\mathcal{S})=\operatorname{tr}\left(\varrho_{\mathcal{S}}\right)=-1$ while for $\chi_{\boldsymbol{R}_{3}}(\mathcal{T})=\operatorname{tr}\left(\varrho_{\mathcal{T}}\right)=0$ due to the identity $1+\omega+\omega^{2}=0$ for $\omega$ third root of identity.

\section{APPENDIX B: SCALAR SECTOR AND THE VACUUM ALIGNMENT}

In this Appendix, we examine the vacuum alignment of the flavon triplets $\Phi$ and $\Omega$ used in the charged lepton and neutrino sectors respectively. As we already mentioned, to achieve the desired vacuum alignments of $\Phi$ and $\Omega$ - the VEV structures that produced the mass matrices (2.10) and (3.7) — we need to avoid any couplings between these flavons triplets. Indeed, this is one of the reasons for adding the $Z_{3}$ symmetry under which the coupling $\Phi \otimes \Omega$ transform as $Q^{2}$. Moreover, it is easy to check from our scalar superfield content in Table III that all trilinear scalar couplings involving $\Phi \otimes \Omega$ are not invariant under the $\boldsymbol{Z}_{3}$ symmetry. The scalar potential of our proposal contains the usual F, D and soft mass terms of the MSSM but this time with the singlet $S$ and the flavons $\chi, \Omega, \Phi$ included. Using Eq. (5.52), the F-term contribution $\left|F_{\text {scal }}\right|^{2}$ to the scalar potential is given by

$$
\begin{aligned}
\mathcal{V}_{F}= & \left|F_{H_{u}}\right|^{2}+\left|F_{H_{d}}\right|^{2}+\left|F_{S}\right|^{2}+\left|F_{\Phi}\right|^{2}+\left|F_{\Omega}\right|^{2}+\left|F_{\chi}\right|^{2} \\
= & \left|\lambda_{1} S H_{d}\right|^{2}+\left|\lambda_{1} S H_{u}\right|^{2}+\left|\lambda_{1} H_{u} H_{d}+\lambda_{2} S^{2}+\lambda_{6} \Omega^{2}\right|^{2} \\
& +\left|\lambda_{4} \Phi^{2}+\mu_{\Phi} \Phi\right|^{2}+\left|\lambda_{3} \Omega^{2}+\lambda_{6} S \Omega\right|^{2}+\left|\lambda_{5} \chi^{2}+\lambda_{7} \Omega^{2}\right|^{2} .
\end{aligned}
$$

On the other hand, as the singlet $S$ and the flavon superfields are gauge singlets, they do not have any D-term contributions to the scalar potential. This restrict the $D$-terms to be exactly the same as in the usual MSSM. 
The final contributions to the scalar potential arising from the soft SUSY breaking terms are given explicitly as

$$
\begin{aligned}
\mathcal{V}_{\text {soft }}= & m_{H_{u}}^{2}\left|H_{u}\right|^{2}+m_{H_{d}}^{2}\left|H_{d}\right|^{2}+m_{s}^{2}|S|^{2} \\
& +m_{\Phi}^{2}|\Phi|^{2}+m_{\Omega}^{2}|\Omega|^{2}+m_{\chi}^{2}|\chi|^{2} \\
& +\left[b_{\Phi} \Phi^{2}+t_{1} S H_{u} H_{d}+t_{2} S^{3}+t_{3} \Omega^{3}+t_{4} \Phi^{3}\right. \\
& \left.+t_{5} \chi^{3}+t_{6} S \Omega^{2}+t_{7} \chi \Omega^{2}+\text { H.c. }\right]
\end{aligned}
$$

where $m_{H_{u}}^{2}, m_{H_{d}}^{2}, m_{s}^{2}, m_{\Phi}^{2}, m_{\Omega}^{2}$, and $m_{\chi}^{2}$ are soft supersymmetry breaking masses, $b_{\Phi}$ is a bilinear soft mass parameter and $t_{i}$ stand for the trilinear coupling between different scalar fields. Now, to study the vacuum alignment of the flavon triplets $\Phi=\varphi_{\Phi}+\theta \psi_{\Phi}+\theta^{2} F_{\Phi}$ and $\Omega=$ $\varphi_{\Omega}+\theta \psi_{\Omega}+\theta^{2} F_{\Omega}$, we denote by $\langle\Phi\rangle=\left(v_{\Phi_{1}}, v_{\Phi_{2}}, v_{\Phi_{3}}\right)$ and $\langle\Omega\rangle=\left(v_{\Omega_{1}}, v_{\Omega_{2}}, v_{\Omega_{3}}\right)$ their VEVs solving the minimum conditions

$$
\frac{\partial \mathcal{V}}{\partial \varphi_{\Phi_{i}}}=0, \quad \frac{\partial \mathcal{V}}{\partial \varphi_{\Omega_{i}}}=0
$$

with $\mathcal{V}=\mathcal{V}_{F}+\mathcal{V}_{\text {soft }}$. We start by minimizing the scalar potential $\mathcal{V}$ with respect to $\varphi_{\Phi}$ where we obtain the following three equations

$$
\begin{aligned}
\left.\frac{\partial \mathcal{V} \Phi)}{\partial \varphi_{\Phi_{1}}}\right|_{\left\langle\Phi_{i}\right\rangle=v_{\Phi_{i}}}= & \lambda_{4}^{2}\left(\frac{52}{9} v_{\Phi_{1}}^{3}+24 v_{\Phi_{1}} v_{\Phi_{2}} v_{\Phi_{3}}+\frac{28}{9} v_{\Phi_{2}}^{3}+\frac{28}{9} v_{\Phi_{3}}^{3}\right)+4 \lambda_{4} b_{\Phi} v_{\Phi_{1}}^{2} \\
& -4 \lambda_{4} b_{\Phi} v_{\Phi_{2}} v_{\Phi_{3}}+2 v_{\Phi_{1}}\left(\mu_{\Phi}^{2}+m_{\Phi}^{2}+2 b_{\Phi}\right)+2 t_{4}\left(v_{\Phi_{1}}^{2}-v_{\Phi_{2}} v_{\Phi_{3}}\right) \\
\left.\frac{\partial \mathcal{V} \Phi)}{\partial \varphi_{\Phi_{2}}}\right|_{\left\langle\Phi_{i}\right\rangle=v_{\Phi_{i}}}= & \lambda_{4}^{2}\left(12 v_{\Phi_{1}}^{2} v_{\Phi_{3}}+\frac{28}{3} v_{\Phi_{1}} v_{\Phi_{2}}^{2}+\frac{44}{3} v_{\Phi_{2}} v_{\Phi_{3}}^{2}\right)+4 \lambda_{4} b_{\Phi} v_{\Phi_{2}}^{2} \\
& -4 \lambda_{4} b_{\Phi} v_{\Phi_{1}} v_{\Phi_{3}}+2 v_{\Phi_{3}}\left(\mu_{\Phi}^{2}+m_{\Phi}^{2}+2 b_{\Phi}\right)+2 t_{4}\left(v_{\Phi_{2}}^{2}-v_{\Phi_{1}} v_{\Phi_{3}}\right) \\
\left.\frac{\partial \mathcal{V} \Phi)}{\partial \varphi_{\Phi_{3}}}\right|_{\left\langle\Phi_{i}\right\rangle=v_{\Phi_{i}}}= & \lambda_{4}^{2}\left(12 v_{\Phi_{1}}^{2} v_{\Phi_{2}}+\frac{28}{3} v_{\Phi_{1}} v_{\Phi_{3}}^{2}+\frac{44}{3} v_{\Phi_{2}}^{2} v_{\Phi_{3}}\right)+4 \lambda_{4} b_{\Phi_{\Phi}} v_{\Phi_{3}}^{2} \\
& -4 \lambda_{4} b_{\Phi} v_{\Phi_{1}} v_{\Phi_{2}}+2 v_{\Phi_{2}}\left(\mu_{\Phi}^{2}+m_{\Phi}^{2}+2 b_{\Phi}\right)+2 t_{4}\left(v_{\Phi_{3}}^{2}-v_{\Phi_{1}} v_{\Phi_{2}}\right)
\end{aligned}
$$

We can approximate the solution of the minimum conditions of $\mathcal{V}$ for the $\mathbb{A}_{4}$ triplet $\Phi$ through the relations

$$
\begin{aligned}
& R_{1}=v_{\Phi_{2}} \frac{\partial \mathcal{V}}{\partial v_{\Phi_{1}}}-v_{\Phi_{1}} \frac{\partial \mathcal{V}}{\partial v_{\Phi_{2}}}=0 \\
& R_{2}=v_{\Phi_{3}} \frac{\partial \mathcal{V}}{\partial v_{\Phi_{1}}}-v_{\Phi_{1}} \frac{\partial \mathcal{V}}{\partial v_{\Phi_{3}}}=0 \\
& R_{3}=v_{\Phi_{3}} \frac{\partial \mathcal{V}}{\partial v_{\Phi_{2}}}-v_{\Phi_{2}} \frac{\partial \mathcal{V}}{\partial v_{\Phi_{3}}}=0
\end{aligned}
$$

where we find

$$
\begin{aligned}
R_{1}= & 4 \lambda_{4}^{2}\left[\frac{13}{9} v_{\Phi_{1}}^{3} v_{\Phi_{2}}+v_{\Phi_{3}}\left(6 v_{\Phi_{1}} v_{\Phi_{2}}^{2}-3 v_{\Phi_{1}}^{3}-\frac{11}{3} v_{\Phi_{1}} v_{\Phi_{2}} v_{\Phi_{3}}\right)\right]+\frac{28}{9} \lambda_{4}^{2} v_{\Phi_{2}}\left(v_{\Phi_{2}}^{3}+v_{\Phi_{3}}^{3}-v_{\Phi_{1}}^{2} v_{\Phi_{2}}\right)+4 \lambda_{4} b_{\Phi}\left(v_{\Phi_{1}}-v_{\Phi_{2}}\right) v_{\Phi_{1}} v_{\Phi_{2}} \\
& +4 \lambda_{4} b_{\Phi}\left(v_{\Phi_{1}}^{2}-v_{\Phi_{2}}^{2}\right) v_{\Phi_{3}}+2 t_{4}\left(v_{\Phi_{1}}^{2}-v_{\Phi_{1}} v_{\Phi_{2}}\right) v_{\Phi_{2}}+2 v_{\Phi_{1}}\left(v_{\Phi_{2}}-v_{\Phi_{3}}\right)\left(\mu_{\Phi}^{2}+m_{\Phi}^{2}+2 b_{\Phi}\right)+2 t_{4}\left(v_{\Phi_{1}}^{2}-v_{\Phi_{2}}^{2}\right) v_{\Phi_{3}} \\
R_{2}= & \frac{4}{9} \lambda_{4}^{2}\left[13 v_{\Phi_{1}}^{3} v_{\Phi_{3}}-v_{\Phi_{2}}\left(27 v_{\Phi_{1}}^{3}+7 v_{\Phi_{2}}^{2} v_{\Phi_{3}}-33 v_{\Phi_{2}} v_{\Phi_{1}} v_{\Phi_{3}}\right)\right]+\frac{4}{9} \lambda_{4}^{2} v_{\Phi_{3}}^{2}\left(7 v_{\Phi_{3}}^{2}+54 v_{\Phi_{1}} v_{\Phi_{2}}-21 v_{\Phi_{1}}^{2}\right)+4 \lambda_{4} b_{\Phi}\left(v_{\Phi_{1}}-v_{\Phi_{3}}\right) v_{\Phi_{1}} v_{\Phi_{3}} \\
& +4 \lambda_{4} b_{\Phi}\left(v_{\Phi_{1}}^{2}-v_{\Phi_{3}}^{2}\right) v_{\Phi_{2}}+2 t_{4}\left(v_{\Phi_{1}}^{2}-v_{\Phi_{3}}^{2}\right) v_{\Phi_{2}}-2 v_{\Phi_{1}}\left(v_{\Phi_{2}}-v_{\Phi_{3}}\right)\left(\mu_{\Phi}^{2}+m_{\Phi}^{2}+2 b_{\Phi}\right)+2 t_{4}\left(v_{\Phi_{1}}^{2}-v_{\Phi_{1}} v_{\Phi_{3}}\right) v_{\Phi_{3}} \\
R_{3}= & \frac{4}{3} \lambda_{4}^{2}\left[9 v_{\Phi_{1}}^{2}\left(v_{\Phi_{3}}^{2}-v_{\Phi_{2}}^{2}\right)-v_{\Phi_{2}}^{2}\left(11 v_{\Phi_{2}} v_{\Phi_{3}}+7 v_{\Phi_{1}} v_{\Phi_{3}}\right)\right]+\frac{4}{3} \lambda_{4}^{2} v_{\Phi_{3}}^{2}\left(11 v_{\Phi_{2}} v_{\Phi_{3}}-7 v_{\Phi_{1}} v_{\Phi_{2}}\right)+4 \lambda_{4} b_{\Phi}\left(v_{\Phi_{1}} v_{\Phi_{2}}-v_{\Phi_{3}}^{2}\right) v_{\Phi_{2}} \\
& +4 \lambda_{4} b_{\Phi}\left(v_{\Phi_{2}}^{2}-v_{\Phi_{1}} v_{\Phi_{3}}\right) v_{\Phi_{3}}+2 t_{4}\left(v_{\Phi_{1}} v_{\Phi_{2}}-v_{\Phi_{3}}^{2}\right) v_{\Phi_{2}}-2\left(v_{\Phi_{2}}^{2}-v_{\Phi_{3}}^{2}\right)\left(\mu_{\Phi}^{2}+m_{\Phi}^{2}+2 b_{\Phi}\right)+2 t_{4}\left(v_{\Phi_{2}}^{2}-v_{\Phi_{1}} v_{\Phi_{3}}\right) v_{\Phi_{3}}
\end{aligned}
$$


Clearly, the only solution for these three equations is the one we have chosen to produce the charged leptons masses (2.11), namely $v_{\Phi_{1}} \neq 0$ and $v_{\Phi_{2}}=v_{\Phi_{3}}=0$. Analogously, we perform the same calculations for the minimum conditions coming from the triplet $\Omega$; we have

$$
\begin{aligned}
\left.\frac{\partial \mathcal{V}(\Omega)}{\partial \varphi_{\Omega_{1}}}\right|_{\left\langle\Omega_{i}\right\rangle=v_{\Omega_{i}}}= & \left(4 \lambda_{2} \lambda_{6} v_{S}^{2}+4 \lambda_{1} \lambda_{6}^{2} v_{d} v_{u}+2 \lambda_{6}^{2} v_{S}^{2}+4 \lambda_{5} \lambda_{7} v_{\varphi}^{2}+2 m_{\Omega}^{2}+2 t_{6} v_{S}+2 t_{7} v_{\varphi}\right) v_{\Omega_{1}} \\
+ & \left(\lambda_{6}^{2}+\lambda_{3}^{2}+\lambda_{7}^{2}\right)\left(\frac{52}{9} v_{\Omega_{1}}^{3}+24 v_{\Omega_{1}} v_{\Omega_{2}} v_{\Omega_{3}}+\frac{28}{9} v_{\Omega_{2}}^{3}+\frac{28}{9} v_{\Omega_{3}}^{3}\right) \\
+ & \left(2 \lambda_{3} \lambda_{6} v_{S}+t_{3}\right)\left(2 v_{\Omega_{1}}^{2}-2 v_{\Omega_{2}} v_{\Omega_{3}}\right) \\
\frac{\partial \mathcal{V}(\Omega)}{\left.\partial \varphi_{\Omega_{2}}\right|_{\left\langle\Omega_{i}\right\rangle=v_{\Omega_{i}}}=} & 2\left(2 \lambda_{2} \lambda_{6} v_{S}^{2}+2 \lambda_{1} \lambda_{6}^{2} v_{d} v_{u}+\lambda_{6}^{2} v_{S}^{2}+2 \lambda_{5} \lambda_{7} v_{\varphi}^{2}+m_{\Omega}^{2}+t_{6} v_{S}+t_{7} v_{\varphi}\right) v_{\Omega_{3}} \\
& +\left(\lambda_{6}^{2}+\lambda_{3}^{2}+\lambda_{7}^{2}\right)\left(12 v_{\Omega_{1}}^{2} v_{\Omega_{3}}+\frac{28}{3} v_{\Omega_{1}} v_{\Omega 2}^{2}+\frac{44}{3} v_{\Omega_{2}} v_{\Omega_{3}}^{2}\right) \\
& +\left(2 \lambda_{3} \lambda_{6} v_{S}+t_{3}\right)\left(2 v_{\Omega_{2}}^{2}-2 v_{\Omega_{1}} v_{\Omega_{3}}\right) \\
\left.\frac{\partial \mathcal{V}(\Omega)}{\partial \varphi_{\Omega_{3}}}\right|_{\left\langle\Omega_{i}\right\rangle=v_{\Omega_{i}}} & 2\left(2 \lambda_{2} \lambda_{6} v_{S}^{2}+2 \lambda_{1} \lambda_{6}^{2} v_{d} v_{u}+\lambda_{6}^{2} v_{S}^{2}+2 \lambda_{5} \lambda_{7} v_{\varphi}^{2}+m_{\Omega_{2}}^{2}+t_{6} v_{S}+t_{7} v_{\varphi}\right) v_{\Omega_{2}} \\
& +\left(\lambda_{6}^{2}+\lambda_{3}^{2}+\lambda_{7}^{2}\right)\left(12 v_{\Omega_{1}}^{2} v_{\Omega_{2}}+\frac{28}{3} v_{\Omega_{1}} v_{\Omega 3}^{2}+\frac{44}{3} v_{\Omega_{2}}^{2} v_{\Omega_{3}}\right) \\
& +\left(2 \lambda_{3} \lambda_{6} v_{S}+t_{3}\right)\left(2 v_{\Omega_{3}}^{2}-2 v_{\Omega_{1}} v_{\Omega_{2}}\right)
\end{aligned}
$$

Similarly to the case of the flavon $\Phi$, we can approach the solution of the minimum conditions of $\mathcal{V}$ for the $\mathbb{A}_{4}$ triplet $\Omega$ through the following equations

$$
\begin{aligned}
& \left(v_{\Omega_{3}} \frac{\partial \mathcal{V}(\Omega)}{\partial \varphi_{\Omega_{1}}}-v_{\Omega_{1}} \frac{\partial \mathcal{V}(\Omega)}{\partial \varphi_{\Omega_{3}}}\right)_{\left\langle\Omega_{i}\right\rangle=v_{\Omega_{i}}}=0 \\
& \left(v_{\Omega_{2}} \frac{\partial \mathcal{V}(\Omega)}{\partial \varphi_{\Omega_{1}}}-v_{\Omega_{1}} \frac{\partial \mathcal{V}(\Omega)}{\partial \varphi_{\Omega_{2}}}\right)_{\left\langle\Omega_{i}\right\rangle=v_{\Omega_{i}}}=0 \\
& \left(v_{\Omega_{3}} \frac{\partial \mathcal{V}(\Omega)}{\partial \varphi_{\Omega_{2}}}-v_{\Omega_{2}} \frac{\partial \mathcal{V}(\Omega)}{\partial \varphi_{\Omega_{3}}}\right)_{\left\langle\Omega_{i}\right\rangle=v_{\Omega_{i}}}=0
\end{aligned}
$$

leading to

$$
\begin{array}{r}
\mathbf{T}_{1}\left(v_{\Omega_{3}}-v_{\Omega_{2}}\right)+\mathbf{T}_{2}\left(v_{\Omega_{1}}-v_{\Omega_{3}}\right)+\mathbf{T}_{3}=0 \\
\mathbf{T}_{1}\left(v_{\Omega_{2}}-v_{\Omega_{3}}\right)+\mathbf{T}_{4}\left(v_{\Omega_{1}}-v_{\Omega_{2}}\right)+\mathbf{T}_{5}=0 \\
\mathbf{T}_{6}\left(v_{\Omega_{3}}^{2}-v_{\Omega_{2}}^{2}\right)+\mathbf{T}_{7}\left(v_{\Omega_{2}}-v_{\Omega_{3}}\right)=0
\end{array}
$$

with

$$
\begin{aligned}
& \mathbf{T}_{1}=2 v_{\Omega_{1}}\left(2 \lambda_{2} \lambda_{6} v_{S}^{2}+2 \lambda_{1} \lambda_{6}^{2} v_{d} v_{u}+\lambda_{6}^{2} v_{S}^{2}+2 \lambda_{5} \lambda_{7} v_{\varphi}^{2}+m_{\Omega}^{2}+t_{6} v_{S}+t_{7} v_{\varphi}\right) \\
& \mathbf{T}_{2}=2\left(2 \lambda_{3} \lambda_{6} v_{S}+t_{3}\right)\left(v_{\Omega_{1}} v_{\Omega_{3}}+v_{\Omega_{2}}\left(v_{\Omega_{1}}+v_{\Omega_{3}}\right)\right) \\
& \mathbf{T}_{3}=\frac{4}{9}\left(\lambda_{6}^{2}+\lambda_{3}^{2}+\lambda_{7}^{2}\right)\left(7 v_{\Omega_{3}}^{4}-21 v_{\Omega_{1}}^{2} v_{\Omega_{3}}^{2}-27 v_{\Omega_{1}}^{3} v_{\Omega_{2}}+13 v_{\Omega_{1}}^{3} v_{\Omega_{3}}+7 v_{\Omega_{2}}^{3} v_{\Omega_{3}}-33 v_{\Omega_{2}}^{2} v_{\Omega_{1}} v_{\Omega_{3}}+54 v_{\Omega_{3}}^{2} v_{\Omega_{1}} v_{\Omega_{2}}\right) \\
& \mathbf{T}_{4}=2\left(2 \lambda_{3} \lambda_{6} v_{S}+t_{3}\right)\left(v_{\Omega_{1}} v_{\Omega_{2}}+v_{\Omega_{3}}\left(v_{\Omega_{1}}+v_{\Omega_{2}}\right)\right)
\end{aligned}
$$


and

$$
\begin{aligned}
\mathbf{T}_{5}= & \frac{4}{9}\left(\lambda_{6}^{2}+\lambda_{3}^{2}+\lambda_{7}^{2}\right)\left(7 v_{\Omega_{2}}^{4}-21 v_{\Omega_{1}}^{2} v_{\Omega_{2}}^{2}+13 v_{\Omega_{1}}^{3} v_{\Omega_{2}}-27 v_{\Omega_{1}}^{3} v_{\Omega_{3}}+7 v_{\Omega_{3}}^{3} v_{\Omega_{2}}-33 v_{\Omega_{3}}^{2} v_{\Omega_{1}} v_{\Omega_{2}}+54 v_{\Omega_{2}}^{2} v_{\Omega_{1}} v_{\Omega_{3}}\right) \\
\mathbf{T}_{6}= & 2\left[2 \lambda_{2} \lambda_{6} v_{S}^{2}+2 \lambda_{1} \lambda_{6}^{2} v_{d} v_{u}+\lambda_{6}^{2} v_{S}^{2}+2 \lambda_{5} \lambda_{7} v_{\varphi}^{2}+m_{\Omega}^{2}+t_{6} v_{S}+t_{7} v_{\varphi}\right. \\
& \left.+2 \lambda_{3} \lambda_{6} v_{S} v_{\Omega_{1}}+t_{3} v_{\Omega_{1}}+\left(\lambda_{6}^{2}+\lambda_{3}^{2}+\lambda_{7}^{2}\right)\left(12 v_{\Omega_{1}}^{2}+\frac{44}{3} v_{\Omega_{2}} v_{\Omega_{3}}\right)\right] \\
\mathbf{T}_{7}= & v_{\Omega_{2}} v_{\Omega_{3}}\left[4 \lambda_{3} \lambda_{6} v_{S}+2 t_{3}+\frac{28}{3}\left(\lambda_{6}^{2}+\lambda_{3}^{2}+\lambda_{7}^{2}\right) v_{\Omega_{1}}\right] .
\end{aligned}
$$

It is clear that the solution for Eq. (B14) is indeed the VEV structure we have chosen to produce the right-handed Majorana neutrinos mass matrix (3.7), namely $v_{\Omega_{1}}=v_{\Omega_{2}}=$ $v_{\Omega_{3}}=v_{\Omega}$.

\section{APPENDIX C: MORE ON FLAVOR SYMMETRY BREAKING OPERATORS}

In this Appendix, we provide a simple list of the five dimensional operators $\mathcal{O}_{5}$ that can be used to break explicitly the discrete $G_{\mathrm{f}}=\mathbb{A}_{4} \times \boldsymbol{Z}_{3}$ flavor group of our model. As before, we focus on the flavor invariant trilinear couplings $\mathcal{W}_{R}=\sum \lambda_{a b c} \Upsilon_{a} \Upsilon_{b} \Upsilon_{c}$ that includes the superfields relevant to the neutrino sector namely

$$
\Upsilon_{a} \Upsilon_{b} \Upsilon_{c} \sim S^{3}, \quad \Omega^{3}, \quad \chi^{3}, \quad S \Omega^{2}, \quad \chi \Omega^{2}, \quad S H_{u} H_{d}
$$

and we would like to look for a chiral superpotential $\mathcal{W}_{\mathrm{NR}}^{\prime}$ made of gauge invariant five dimensional operators type $\frac{1}{M_{\mathrm{Pl}}^{2}} \mathcal{O}_{5}$ that breaks explicitly the full or a part of the flavor symmetry $G_{\mathrm{f}}$. Clearly one can write down a big list of such gauge symmetric operators; a remarkable family of gauge invariant five dimensional operators $\mathcal{O}_{5}$ that breaks the flavor symmetry $G_{\mathrm{f}}$ is obtained by thinking of $\mathcal{O}_{5}$ as follows

$$
\mathcal{O}_{5} \sim \mathcal{O}_{2} \times \Upsilon_{a} \Upsilon_{b} \Upsilon_{c}
$$

with $\mathcal{O}_{2}$ breaking $G_{\mathrm{f}}$ partially or completely. Moreover, seen that $G_{\mathrm{f}}$ is the product of two factors, the breaking may be carried either by one of the two factors; that is $\mathbb{A}_{4}$ or by $Z_{3}$; or by both of them. ${ }^{13}$ For example, candidates for $\mathcal{O}_{2}$ breaking $\boldsymbol{Z}_{3}$ are directly read from the Table III; they are given by the following quadratic chiral superfield monomials

$$
\mathcal{O}_{2}: S^{2}, \quad \Omega^{2}, \quad \chi^{2}, \quad H_{u} H_{d}
$$

From these four quadratic candidates, only $S^{2}$ breaks both $\mathbb{A}_{4}$ and $\boldsymbol{Z}_{3}$ symmetries. Hence, a simple candidate form for the superpotential $\mathcal{W}_{\mathrm{NR}}^{\prime}$ reads therefore as follows

$\mathcal{W}_{\mathrm{NR}}^{\prime}=\frac{1}{M_{\mathrm{Pl}}^{2}}\left[h_{i} H_{u} H_{d}+h_{i}^{\prime} S^{2}+l_{i} \Omega^{2}+l_{i}^{\prime} \chi^{2}\right] \times \mathcal{W}_{R}$

where $\mathcal{W}_{R}=\sum \lambda_{a b c} \Upsilon_{a} \Upsilon_{b} \Upsilon_{c}$ and where $l_{3} \equiv \lambda_{3}^{\prime}$ according to Eq. (5.53). From this $\mathcal{W}_{\mathrm{NR}}^{\prime}$, one can compute the contribution to the effective scalar potential $\mathcal{V}$ that breaks the flavor symmetry. In what follows, we illustrate the calculation leading to the scalar potential for the very particular and simple example where $\mathcal{W}_{\mathrm{NR}}^{\prime}$ is restricted to the operator of Eq. (5.53) namely

$$
\mathcal{W}_{\mathrm{NR}}=\left.\frac{\lambda_{3}^{\prime}}{M_{\mathrm{Pl}}^{2}}\left(\Omega^{5}\right)\right|_{(1, \omega)}
$$

By using the Feynman rules for supergraphs of chiral superfields; in particular for the chiral $S=S_{s}+\theta S_{\psi}+\theta^{2} S_{F}$, the contribution of the supergraph in Fig. 12 to the effective action is given by $[117,118,125,126]$

$$
\begin{aligned}
\delta S \approx & \frac{\lambda_{3}^{2} \lambda_{3}^{\prime}}{M_{\mathrm{Pl}}^{2}} \int d^{4} x_{1} d^{4} x_{2} d^{4} x_{3} d^{4} \theta_{1} d^{4} \theta_{2} d^{4} \theta_{3} \Omega\left(x_{1}, \theta_{1}\right) \frac{1}{\overline{\boldsymbol{\phi}}\left(\bar{\theta}_{1}\right)} e^{\frac{2 K_{(12)}}{3}} e^{\frac{K_{(23)}}{3}} e^{\frac{2 K_{(13)}}{3}} \\
& \times\left(\frac{\bar{D}_{1}^{2}}{4 \square_{1}} \delta_{12}\right)\left(\frac{D_{2}^{2} \bar{D}_{2}^{2}}{16 \square_{2}} \delta_{12}\right)\left(\frac{D_{2}^{2}}{4 \square_{2}} \delta_{23}\right)\left(\frac{\bar{D}_{3}^{2}}{4 \square_{3}} \delta_{13}\right)\left(\frac{\bar{D}_{3}^{2} D_{3}^{2}}{16 \square_{3}} \delta_{13}\right)
\end{aligned}
$$

\footnotetext{
${ }^{13}$ For the contributions that arise from the combination between two different fields, namely $S, \Omega$, and $\chi$, they can be eliminated by the same R-symmetries usually used to avoid the existence of terms of order four [119,120].
} 
where

$$
\delta_{i j}=\delta^{4}\left(x_{i}-x_{j}\right) \delta^{4}\left(\theta_{i}-\theta_{j}\right),
$$

the compensator superfield is given by $\overline{\boldsymbol{\phi}}\left(\bar{\theta}_{1}\right)=1+\bar{\theta}_{1}^{2} \frac{M_{s}^{2}}{M_{\mathrm{PI}}}$ and $K_{(i j)}=K\left(\theta_{i}, \bar{\theta}_{j}\right)$ which is the minimal Kahler potential of the model defined as $K=\Sigma \bar{\Sigma}$ where $\Sigma$ stands for chiral superfields of the model. One can evaluate this expression by integrating by parts and use the factors of $\delta^{4}\left(\theta_{i}-\theta_{j}\right)$ to eliminate $\theta$ integrals. Moreover, by using the algebra of the supersymmetric covariant derivatives we can remove $D^{2} \bar{D}^{2}$ due to properties $[117,118,121,125,126]$ like

$$
D^{2} \bar{D}^{2} D^{2}=16 \square D^{2} ; \quad \bar{D}^{2} D^{2} \bar{D}^{2}=16 \square \bar{D}^{2}
$$

and

$$
\int d^{2} \theta_{2} \delta^{4}\left(\theta_{2}-\theta_{1}\right) D^{2} \bar{D}^{2} \delta^{4}\left(\theta_{2}-\theta_{1}\right)=16
$$

In doing so, the integral (C6) gets reduced to a single integral over $\theta_{1}$ of the form

$$
\delta S \approx \frac{\lambda_{3}^{2} \lambda_{3}^{\prime}}{M_{\mathrm{Pl}}^{2}} \int d^{4} x_{1} d^{4} \theta_{1} \Omega\left(x_{1}, \theta_{1}\right) \frac{1}{\bar{\phi}\left(\bar{\theta}_{1}\right)}\left(\frac{\bar{D}^{2}}{4}\right) e^{\frac{5 K_{(11)}}{3}} \times \mathcal{I}_{3}
$$

with

$$
\begin{aligned}
\mathcal{I}_{3} & =\int \frac{d^{4} k_{1}}{(2 \pi)^{4}} \frac{d^{4} k_{2}}{(2 \pi)^{4}} \frac{d^{4} k_{3}}{(2 \pi)^{4}} \frac{1}{k_{1}^{2} k_{2}^{2} k_{3}^{2}\left(k_{1}-k_{2}\right)^{2}\left(k_{1}-k_{3}\right)^{2}} \\
& \sim \mathcal{O}\left(\frac{M_{P}^{2}}{\left(16 \pi^{2}\right)^{3}}\right)
\end{aligned}
$$

Using the following relations $[117,118,125,126]$

$$
\begin{aligned}
\exp \left(\frac{2 K}{3 M_{\mathrm{Pl}}^{2}}\right) & \approx 1+\theta^{2} \frac{M_{s}^{2}}{M_{\mathrm{Pl}}}+\bar{\theta}^{2} \frac{M_{s}^{2}}{M_{\mathrm{Pl}}}+\theta^{2} \bar{\theta}^{2} \frac{M_{s}^{4}}{M_{\mathrm{Pl}}^{2}} \quad \text { and } \\
\phi & \approx 1+\theta^{2} \frac{M_{s}^{2}}{M_{\mathrm{Pl}}}
\end{aligned}
$$

which are respectively the classical VEVs for the Kahler potential and the nonpropagating compensator superfield, we get an additional contribution to the effective potential; it is given by

$$
\delta \mathcal{V}_{\text {eff }}=\frac{\lambda_{3}^{2} \lambda_{3}^{\prime} M_{W}^{3}}{\left(16 \pi^{2}\right)^{3}}\left(\phi_{\Omega}+\bar{\phi}_{\Omega}\right)+\frac{\lambda_{2}^{2} \lambda_{5}^{\prime} M_{W}^{2}}{\left(16 \pi^{2}\right)^{3}}\left(\mathrm{~F}_{\Omega}+\overline{\mathrm{F}}_{\Omega}\right)
$$

where the linear term proportional to $M_{W}^{3}$ is the contribution used to break explicitly the full flavor symmetry $G_{\mathrm{f}}=\mathbb{A}_{4} \times \mathbf{Z}_{3}$ of our model.

\section{APPENDIX D: DOMAIN WALLS FROM BREAKING $\mathbb{A}_{\mathbf{4}}$ BY $\langle S\rangle$}

In this Appendix, we describe the case where the breaking pattern in the neutrino sector is driven by the VEV of the NMSSM singlet $S$. Since $S$ is invariant under the actions of $\mathcal{S}, \mathcal{S}^{\prime}$, and $\mathcal{S}^{\prime \prime},\langle S\rangle$ breaks $G_{\mathrm{f}}$ down to its Klein subgroup $\mathbb{V}_{4}$ with the broken part is given by $\mathbb{Z}_{3}$. The analogue of the $\vartheta_{\alpha}^{ \pm}$- vacua (5.48) are now given by three degenerate $\langle S\rangle_{i}$ rotated by $\mathbb{Z}_{3}$ that we denote like

$$
\zeta_{1}=v_{S} X_{4}, \quad \zeta_{2}=\omega v_{S} X_{4}, \quad \zeta_{3}=\bar{\omega} v_{S} X_{4}
$$

where $\omega=e^{\frac{2 i \pi}{3}}$ and where $X_{4}$ is as in (5.1). These vacua can be obtained by starting from $\zeta_{1}$ and acting by $\mathcal{T}$ generator of $\mathbb{Z}_{3}$. They obey the property

$$
\sum_{\alpha=1}^{3} \zeta_{\alpha}=0
$$

and so can be represented by a $2 \mathrm{~d}$ triangle. Indeed, by using the real basis vector (5.3); we can express the $X_{4}^{T}$ and $i \otimes X_{4}^{T}$ directions respectively like $(1,0)$ and $(0,1)$; then substituting back into the above vacua, we obtain after setting $\vec{\zeta}_{i}=\left(\operatorname{Re} \zeta_{i}, \operatorname{Im} \zeta_{i}\right)$ the following real VEVs

$$
\vec{\zeta}_{1}=\left(\begin{array}{c}
v_{S} \\
0
\end{array}\right), \quad \vec{\zeta}_{2}=\left(\begin{array}{c}
-\frac{v_{S}}{2} \\
\frac{\sqrt{3}}{2} v_{S}
\end{array}\right), \quad \vec{\zeta}_{3}=\left(\begin{array}{c}
-\frac{v_{S}}{2} \\
-\frac{\sqrt{3}}{2} v_{S}
\end{array}\right)
$$

defining the vertices of a triangle quiver diagram given by Fig. 13 representing as well the DWs extending between the three vertices. The nonrenormalizable operators $\mathcal{W}_{\mathrm{NR}}$ of order five operators that can break explicitly the full $\mathbb{A}_{4} \times$ $Z_{3}$ flavor symmetry down to $\mathbb{V}_{4}$ is given by the following operator

$$
\mathcal{W}_{\mathrm{NR}}=\frac{\lambda_{5}^{\prime}}{M_{\mathrm{Pl}}^{2}} S^{5}
$$

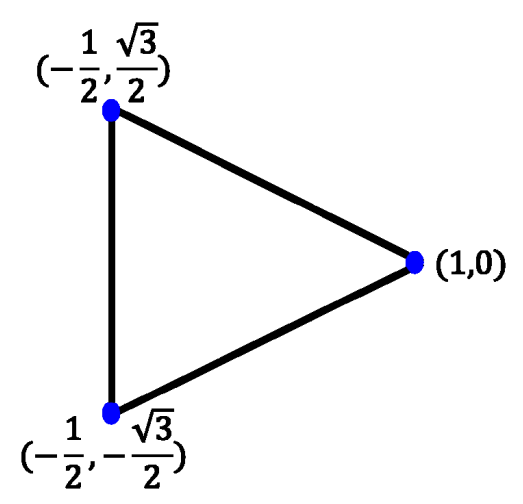

FIG. 13. A quiver diagram representing domain walls using characters of the generator of $\mathbb{Z}_{3}$. The vertices correspond to the three vacua, while the domain walls are represented by line segments (edges) interpolating between the vacua. 
[1] S. Fukuda et al. (Super-Kamiokande Collaboration), Constraints on Neutrino Oscillations Using 1258 Days of Super-Kamiokande Solar Neutrino Data, Phys. Rev. Lett. 86, 5656 (2001).

[2] Q. R. Ahmad et al. (SNO Collaboration), Direct Evidence for Neutrino Flavor Transformation from Neutral-Current Interactions in the Sudbury Neutrino Observatory, Phys. Rev. Lett. 89, 011301 (2002).

[3] Q. R. Ahmad et al. (SNO Collaboration), Measurement of Day and Night Neutrino Energy Spectra at SNO and Constraints on Neutrino Mixing Parameters, Phys. Rev. Lett. 89, 011302 (2002).

[4] G. Aad et al. (ATLAS Collaboration), Observation of a new particle in the search for the standard model Higgs boson with the ATLAS detector at the LHC, Phys. Lett. B 716, 1 (2012).

[5] CMS Collaboration, Observation of a new boson at a mass of $125 \mathrm{GeV}$ with the CMS experiment at the LHC, Phys. Lett. B 716, 30 (2012).

[6] P. Fayet, Supergauge invariant extension of the Higgs mechanism and a model for the electron and its neutrino, Nucl. Phys. B90, 104 (1975).

[7] P. Fayet, Supersymmetry and weak, electromagnetic and strong interactions, Phys. Lett. 64B, 159 (1976).

[8] P. Fayet, Spontaneously broken supersymmetric theories of weak, electromagnetic and strong interactions, Phys. Lett. 69B, 489 (1977).

[9] J.E. Kim and H. P. Nilles, The $\mu$ problem and the strong CP problem, Phys. Lett. 138B, 150 (1984).

[10] H. P. Nilles, M. Srednicki, and D. Wyler, Weak interaction breakdown induced by supergravity, Phys. Lett. 120B, 346 (1983).

[11] J. M. Frere, D. R. T. Jones, and S. Raby, Fermion masses and induction of the weak scale by supergravity, Nucl. Phys. B222, 11 (1983).

[12] H. P. Nilles, Supersymmetry, supergravity and particle physics, Phys. Rep. 110, 1 (1984).

[13] U. Ellwanger, M. Rausch de Traubenberg, and C. A. Savoy, Phenomenology of supersymmetric models with a singlet, Nucl. Phys. B492, 2 (1997).

[14] J. R. Ellis, K. Enqvist, D. V. Nanopoulos, K. A. Olive, M. Quiros, and F. Zwirner, Observables in lowenergy superstring models, Phys. Lett. B 176, 403 (1986).

[15] Ya. B. Zel'dovich, I. Yu. Kobzarev, and L. B. Okun, Cosmological consequences of a spontaneous breakdown of a discrete symmetry, Sov. Phys. JETP 40, 1 (1975).

[16] T. W. B. Kibble, Topology of cosmic domains and strings, J. Phys. A 9, 1387 (1976).

[17] T. W. B. Kibble, Some implications of a cosmological phase transition, Phys. Rep. 67, 183 (1980).

[18] O. Bertolama and G. G. Ross, Inflation as a cure for the cosmological problems of superstring models with intermediate scale breaking, Phys. Lett. B 183, 163 (1987).

[19] J. Preskill, S. P. Trivedi, F. Wilczek, and M. B. Wise, Cosmology and broken discrete symmetry, Nucl. Phys. B363, 207 (1991).

[20] B. Ray and G. Senjanovic, Gravity and the domain-wall problem, Phys. Rev. D 49, 2729 (1994).
[21] T. Moroi and K. Nakayama, Domain walls and gravitational waves after thermal inflation, Phys. Lett. B 703, 160 (2011).

[22] K. Kadota, M. Kawasaki, and K. Saikawa, Gravitational waves from domain walls in the next-to-minimal supersymmetric standard model, J. Cosmol. Astropart. Phys. 10 (2015) 041.

[23] T. Krajewski, Z. Lalak, M. Lewicki, and P. Olszewski, Domain walls and gravitational waves in the standard model, J. Cosmol. Astropart. Phys. 12 (2016) 036.

[24] B. P. Abbott et al. (Virgo and LIGO Scientific Collaborations), Observation of Gravitational Waves from a Binary Black Hole Merger, Phys. Rev. Lett. 116, 061102 (2016).

[25] I. Esteban, M. C. Gonzalez-Garcia, M. Maltoni, I. Martinez-Soler, and T. Schwetz, Updated fit to three neutrino mixing: Exploring the accelerator-reactor complementarity, J. High Energy Phys. 01 (2017) 087.

[26] F. Capozzi, E. Di Valentino, E. Lisi, A. Marrone, A. Melchiorri, and A. Palazzo, Global constraints on absolute neutrino masses and their ordering, Phys. Rev. D 95, 096014 (2017).

[27] P. F. de Salas, D. V. Forero, C. A. Ternes, M. Tortola, and J. W. F. Valle, Status of neutrino oscillations 2018: $3 \sigma$ hint for normal mass ordering and improved $C P$ sensitivity, Phys. Lett. B 782, 633 (2018).

[28] F. Capozzi, E. Lisi, A. Marrone, and A. Palazzo, Current unknowns in the three neutrino framework, Prog. Part. Nucl. Phys. 102, 48 (2018).

[29] I. Esteban, M. C. Gonzalez-Garcia, A. Hernandez-Cabezudo, M. Maltoni, and T. Schwetz, Global analysis of three-flavour neutrino oscillations: Synergies and tensions in the determination of $\theta_{23}, \delta_{C P}$, and the mass ordering, J. High Energy Phys. 01 (2019) 106.

[30] P. Minkowski, $\mu \rightarrow e \gamma$ at a Rate of One Out of 109 Muon Decays?, Phys. Lett. 67B, 421 (1977).

[31] J. Schechter and J.W.F. Valle, Neutrino masses in $S U(2) \otimes U(1)$ theories, Phys. Rev. D 22, 2227 (1980).

[32] M. Gell-Mann, P. Ramond, and R. Slansky, Complex spinors and unified theories, Conf. Proc. C790927, 315 (1979).

[33] T. Yanagida, Horizontal gauge symmetry and masses of neutrinos, Conf. Proc. C7902131, 95 (1979).

[34] R. N. Mohapatra and G. Senjanovic, Neutrino Mass and Spontaneous Parity Nonconservation, Phys. Rev. Lett. 44, 912 (1980).

[35] J. Schechter and J. W. F. Valle, Neutrino decay and spontaneous violation of lepton number, Phys. Rev. D 25, 774 (1982).

[36] P. F. Harrison, D. H. Perkins, and W. G. Scott, Tri-bimaximal mixing and the neutrino oscillation data, Phys. Lett. B 530, 167 (2002).

[37] Y. Abe et al. (Double-Chooz Collaboration), Indication for the Disappearance of Reactor Electron Antineutrinos in the Double Chooz Experiment, Phys. Rev. Lett. 108, 131801 (2012).

[38] F. P. An et al. (Daya-Bay Collaboration), Observation of Electron-Antineutrino Disappearance at Daya Bay, Phys. Rev. Lett. 108, 171803 (2012). 
[39] J. K. Ahn et al. (RENO Collaboration), Observation of Reactor Electron Antineutrinos Disappearance in the RENO Experiment, Phys. Rev. Lett. 108, 191802 (2012).

[40] S. F. King, Unified models of neutrinos, flavour and $C P$ Violation, Prog. Part. Nucl. Phys. 94, 217 (2017).

[41] F. Plentinger and W. Rodejohann, Deviations from tribimaximal neutrino mixing, Phys. Lett. B 625, 264 (2005).

[42] S. Antusch and S. F. King, Charged lepton corrections to neutrino mixing angles and $C P$ phases revisited, Phys. Lett. B 631, 42 (2005).

[43] S. Luo and Z. Z. Xing, Generalized tri-bimaximal neutrino mixing and its sensitivity to radiative corrections, Phys. Lett. B 632, 341 (2006).

[44] N. Haba, A. Watanabe, and K. Yoshioka, Twisted Flavors and Tribimaximal Neutrino Mixing, Phys. Rev. Lett. 97, 041601 (2006).

[45] M. Hirsch, E. Ma, J. C. Romao, J. W. F. Valle, and A. Villanova del Moral, Minimal supergravity radiative effects on the tribimaximal neutrino mixing pattern, Phys. Rev. D 75, 053006 (2007).

[46] X.G. He and A. Zee, Minimal modification to the tri-bimaximal neutrino mixing, Phys. Lett. B 645, 427 (2007).

[47] A. Dighe, S. Goswami, and W. Rodejohann, Corrections to tribimaximal neutrino mixing: Renormalization and Planck scale effects, Phys. Rev. D 75, 073023 (2007).

[48] S. Antusch, P. Huber, S. F. King, and T. Schwetz, Neutrino mixing sum rules and oscillation experiments, J. High Energy Phys. 04 (2007) 060.

[49] M. Lindner and W. Rodejohann, Large and almost maximal neutrino mixing within the type II see-saw mechanism, J. High Energy Phys. 05 (2007) 089.

[50] K. A. Hochmuth, S. T. Petcov, and W. Rodejohann, $U_{\text {PMNS }}=U_{l}^{\dagger} U_{\nu}$, Phys. Lett. B 654, 177 (2007).

[51] S. Pakvasa, W. Rodejohann, and T. J. Weiler, Unitary Parametrization of Perturbations to Tribimaximal Neutrino Mixing, Phys. Rev. Lett. 100, 111801 (2008).

[52] C. H. Albright and W. Rodejohann, Comparing trimaximal mixing and its variants with deviations from tri-bimaximal mixing, Eur. Phys. J. C 62, 599 (2009).

[53] W. Rodejohann, Deviations from tri-bimaximal mixing, Nucl. Phys. B188, 336 (2009).

[54] J. Barry and W. Rodejohann, Deviations from tribimaximal mixing due to the vacuum expectation value misalignment in $A_{4}$ models, Phys. Rev. D 81, 093002 (2010).

[55] E. W. Otten and C. Weinheimer, Neutrino mass limit from tritium $\beta$ decay, Rep. Prog. Phys. 71, 086201 (2008).

[56] G. Drexlin, V. Hannen, S. Mertens, and C. Weinheimer, Current direct neutrino mass experiments, Adv. High Energy Phys. 2013, 293986 (2013).

[57] S. M. Bilenky and C. Giunti, Neutrinoless double-beta decay: A probe of physics beyond the standard model, Int. J. Mod. Phys. A 30, 1530001 (2015).

[58] H. Päs and W. Rodejohann, Neutrinoless double beta decay, New J. Phys. 17, 115010 (2015).

[59] S. Dell'Oro, S. Marcocci, M. Viel, and F. Vissani, Neutrinoless double beta decay: 2015 review, Adv. High Energy Phys. 2016, 2162659 (2016).
[60] J. D. Vergados, H. Ejiri, and F. Simkovic, Neutrinoless double beta decay and neutrino mass, Int. J. Mod. Phys. E 25, 1630007 (2016).

[61] R. Henning, Current status of neutrinoless double-beta decay searches, Rev. Phys. 1, 29 (2016).

[62] P. A. R. Ade et al. (Planck Collaboration), Planck 2015 results XIII. Cosmological parameters, Astron. Astrophys. 594, A13 (2016).

[63] G. Altarelli and F. Feruglio, Tri-bimaximal neutrino mixing, $A_{4}$ and the modular symmetry, Nucl. Phys. B741, 215 (2006).

[64] M. Maniatis, The next-to-minimal supersymmetric extension of the standard model reviewed, Int. J. Mod. Phys. A 25, 3505 (2010).

[65] B. Brahmachari, S. Choubey, and M. Mitra, $A_{4}$ flavor symmetry and neutrino phenomenology, Phys. Rev. D 77, 073008 (2008).

[66] E. Ma and D. Wegman, Nonzero $\theta_{13}$ for Neutrino Mixing in the Context of $A_{4}$ Symmetry, Phys. Rev. Lett. 107, 061803 (2011).

[67] T. Fukuyama and H. Nishiura, Proceeding of 1997 Shizuoka Workshop on Masses and Mixings of Quarks and Leptons (World Scientific, Singapore, 1997).

[68] Z.z. Xing and Z.h. Zhao, A review of $\mu-\tau$ flavor symmetry in neutrino physics, Rep. Prog. Phys. 79, 076201 (2016).

[69] W. Grimus and L. Lavoura, A model for trimaximal lepton mixing, J. High Energy Phys. 09 (2008) 106.

[70] H. Ishimori, Y. Shimizu, M. Tanimoto, and A. Watanabe, Neutrino masses and mixing from flavor twisting, Phys. Rev. D 83, 033004 (2011).

[71] Y. Shimizu, M. Tanimoto, and A. Watanabe, Breaking tribimaximal mixing and large $\theta_{13}$, Prog. Theor. Phys. 126, 81 (2011).

[72] X. G. He and A. Zee, Minimal modification to tribimaximal mixing, Phys. Rev. D 84, 053004 (2011).

[73] I. de M. Varzielas and D. Pidt, UV completions of flavour models and large $\theta_{13}$, J. High Energy Phys. 03 (2013) 065.

[74] G. B. Gelmini, M. Gleiser, and E. W. Kolb, Cosmology of biased discrete symmetry breaking, Phys. Rev. D 39, 1558 (1989).

[75] S. A. Abel, S. Sarkar, and P. L. White, On the cosmological domain wall problem for the minimally extended supersymmetric standard model, Nucl. Phys. B454, 663 (1995).

[76] R. Ahl Laamara, M. A. Loualidi, and E. H. Saidi, Type II seesaw supersymmetric neutrino model for $\theta_{13} \neq 0$, Phys. Rev. D 93, 113005 (2016).

[77] E. H. Saidi, On building superpotentials in F-GUTs, Prog. Theor. Exp. Phys. 2016, 013B07 (2016).

[78] M. Tanabashi et al. (Particle Data Group), APS: Review of particle physics, Phys. Rev. D 98, 030001 (2018).

[79] E. Ma, $A_{4}$ origin of the neutrino mass matrix, Phys. Rev. D 70, 031901 (2004).

[80] G. Altarelli and F. Feruglio, Tri-bimaximal neutrino mixing from discrete symmetry in extra dimensions, Nucl. Phys. B720, 64 (2005).

[81] P. Adamson et al. (NOvA Collaboration), Measurement of the Neutrino Mixing Angle in NOvA, Phys. Rev. Lett. 118, 151802 (2017). 
[82] M. A. Acero et al. (NOvA Collaboration), New constraints on oscillation parameters from e appearance and disappearance in the NOvA experiment, Phys. Rev. D 98, 032012 (2018).

[83] S. K. Kang, Y. Shimizu, K. Takagi, S. Takahashi, and M. Tanimoto, Revisiting $A_{4}$ model for leptons in light of NuFIT 3.2, Prog. Theor. Exp. Phys. 2018, 083 B01 (2018).

[84] S. F. King and C. Luhn, Trimaximal neutrino mixing from vacuum alignment in $A_{4}$ and $S_{4}$ models, J. High Energy Phys. 09 (2011) 042.

[85] S.T. Petcov, Discrete flavour symmetries, neutrino mixing and leptonic $C P$ violation, Eur. Phys. J. C 78, 709 (2018).

[86] Rui-Yun Guo, Jing-Fei Zhang, and Xin Zhang, Exploring neutrino mass and mass hierarchy in the scenario of vacuum energy interacting with cold dark matter, Chin. Phys. C 42, 095103 (2018).

[87] N. Palanque-Delabrouille et al., Neutrino masses and cosmology with Lyman-alpha forest power spectrum, J. Cosmol. Astropart. Phys. 11 (2015) 011.

[88] E. Di Valentino, E. Giusarma, O. Mena, A. Melchiorri, and J. Silk, Cosmological limits on neutrino unknowns versus low redshift priors, Phys. Rev. D 93, 083527 (2016).

[89] A. J. Cuesta, V. Niro, and L. Verde, Neutrino mass limits: Robust information from the power spectrum of galaxy surveys, Phys. Dark Universe 13, 77 (2016).

[90] S. Vagnozzi, E. Giusarma, O. Mena, K. Freese, M. Gerbino, S. Ho, and M. Lattanzi, Unveiling secrets with cosmological data: Neutrino masses and mass hierarchy, Phys. Rev. D 96, 123503 (2017).

[91] R. Emami et al., Evidence of neutrino enhanced clustering in a complete sample of Sloan survey clusters, implying $\sum m_{\nu}=0.11 \pm 0.03 \mathrm{eV}$, arXiv:1711.05210.

[92] S. Mertens, Status of the KATRIN experiment and prospects to search for keV-mass sterile neutrinos in Tritium $\beta$-decay, Phys. Procedia 61, 267 (2015).

[93] B. Pontecorvo, Zh. Eksp. Teor. Fiz. 33, 549 (1957) [Sov. Phys. JETP 6, 429 (1957)]; Neutrino experiments and the problem of conservation of leptonic charge, Sov. Phys. JETP 26, 984 (1968); Z. Maki, M. Nakagawa, and S. Sakata, Remarks on the unified model of elementary particles, Prog. Theor. Phys. 28, 870 (1962).

[94] A. A. Esfahani et al. Project 8 Collaboration, Determining the neutrino mass with cyclotron radiation emission spectroscopy—Project 8, J. Phys. G 44, 054004 (2017).

[95] L. Canonica et al. (CUORE Collaboration), Status and prospects for CUORE, J. Phys. Conf. Ser. 888, 012034 (2017).

[96] M. Auger et al. (EXO-200 Collaboration), Search for Neutrinoless Double-Beta Decay in ${ }^{136}$ Xe with EXO-200, Phys. Rev. Lett. 109, 032505 (2012).

[97] J. J. Gomez-Cadenas et al. (NEXT Collaboration), Present status and future perspectives of the NEXT experiment, Adv. High Energy Phys. 2014, 907067 (2014).

[98] A. Barabash (SuperNEMO Collaboration), SuperNEMO double beta decay experiment, J. Phys. Conf. Ser. 375, 042012 (2012).

[99] M. Agostini et al. (GERDA Collaboration), Results on Neutrinoless Double- $\beta$ Decay of ${ }^{76} \mathrm{Ge}$ from Phase I of the GERDA Experiment, Phys. Rev. Lett. 111, 122503 (2013).

[100] A. Gando et al. (KamLAND-Zen Collaboration), Search for Majorana Neutrinos Near the Inverted Mass Hierarchy Region with KamLAND-Zen, Phys. Rev. Lett. 117, 082503 (2016).

[101] W. Maneschg, Present status of neutrinoless double beta decay searches, arXiv:1704.08537.

[102] A. Pocar (EXO-200 and nEXO Collaborations), Searching for neutrino-less double beta decay with EXO-200 and nEXO, Nucl. Part. Phys. Proc. 42, 265 (2015).

[103] T. Brunner and L. Winslow, Searching for $0 \nu \beta \beta$ decay in ${ }^{136} \mathrm{Xe}$ : Toward the ton-scale and beyond, Nucl. Phys. News 27, 14 (2017).

[104] M. Agostini et al. (GERDA Collaboration), Backgroundfree search for neutrinoless double- $\beta$ decay of ${ }^{76} \mathrm{Ge}$ with GERDA, Nature (London) 544, 47 (2017).

[105] F. Riva, Low-scale leptogenesis and the domain wall problem in models with discrete flavor symmetries, Phys. Lett. B 690, 443 (2010).

[106] S. Antusch and D. Nolde, Matter inflation with $A_{4}$ flavour symmetry breaking, J. Cosmol. Astropart. Phys. 10 (2013) 028.

[107] S. Chigusa and K. Nakayama, Anomalous discrete flavor symmetry and domain wall problem, Phys. Lett. B 788, 249 (2019).

[108] S. F. King and Y. L. Zhou, Spontaneous breaking of $S O(3)$ to finite family symmetries with supersymmetry-An $A_{4}$ model, J. High Energy Phys. 11 (2018) 173.

[109] S. Chigusa, S. Kasuya, and K. Nakayama, Flavon stabilization in models with discrete flavor symmetry, Phys. Lett. B 788, 494 (2019).

[110] M. Dine, F. Takahashi, and T. T. Yanagida, Discrete R symmetries and domain walls, J. High Energy Phys. 07 (2010) 003.

[111] D. Larson et al., Seven-year Wilkinson microwave anisotropy probe (WMAP) observations: Power spectra and WMAP-derived parameters, Astrophys. J. Suppl. Ser. 192, 16 (2011).

[112] E. Komatsu et al. (WMAP Collaboration), Seven-year Wilkinson Microwave Anisotropy Probe (WMAP) observations: Cosmological interpretation, Astrophys. J. Suppl. Ser. 192, 18 (2011).

[113] A. Vilenkin, Cosmic strings and domain walls, Phys. Rep. 121, 263 (1985).

[114] P. Sikivie, Axions, Domain Walls, and the Early Universe, Phys. Rev. Lett. 48, 1156 (1982).

[115] G. B. Gelmini, M. Gleiser, and E. W. Kolb, Cosmology of biased discrete symmetry breaking, Phys. Rev. D 39, 1558 (1989).

[116] S.E. Larsson, S. Sarkar, and P.L. White, Evading the cosmological domain wall problem, Phys. Rev. D 55, 5129 (1997).

[117] J. Bagger and E. Poppitz, Destabilizing Divergences in Supergravity-Coupled Supersymmetric Theories, Phys. Rev. Lett. 71, 2380 (1993).

[118] J. Bagger, E. Poppitz, and L. Randall, Destabilizing divergences in supergravity theories at two loops, Nucl. Phys. B455, 59 (1995). 
[119] C. Panagiotakopoulos and K. Tamvakis, Stabilized NMSSM without domain walls, Phys. Lett. B 446, 224 (1999).

[120] C. Panagiotakopoulos and K. Tamvakis, New minimal extension of MSSM, Phys. Lett. B 469, 145 (1999).

[121] S. A. Abel, Destabilizing divergences in the NMSSM, Nucl. Phys. B480, 55 (1996).

[122] J. Wess and J. Bagger, Supersymmetry and Supergravity (Princeton University Press, Princeton, NJ, 1992).
[123] S. J. Gates, Jr., M. T. Grisaru, M. Rocek, and W. Siegel, Superspace, or one thousand and one lessons in supersymmetry, Front. Phys. 58, 1 (1983).

[124] P. P. Srivastava, Supersymmetry, Superfields and Supergravity: An Introduction (Adam Hilger, Bristol, UK, 1986).

[125] U. Ellwanger, Nonrenormalizable interactions from supergravity, quantum corrections and effective low-energy theories, Phys. Lett. 133B, 187 (1983).

[126] V. Jain, On destabilizing divergencies in supergravity models, Phys. Lett. B 351, 481 (1995). 